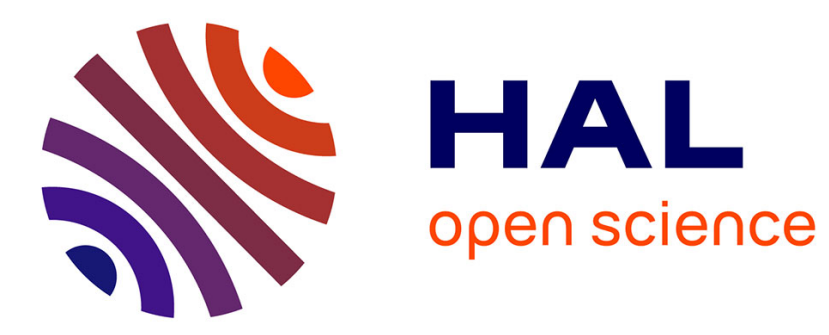

\title{
Hybrid high-order methods for the acoustic wave equation in the time domain
}

Erik Burman, Omar Duran, Alexandre Ern

\section{To cite this version:}

Erik Burman, Omar Duran, Alexandre Ern. Hybrid high-order methods for the acoustic wave equation in the time domain. Communications on Applied Mathematics and Computation, 2022, 4 (2), pp.597633. hal-02922702v2

\section{HAL Id: hal-02922702 \\ https://hal.science/hal-02922702v2}

Submitted on 8 Mar 2021

HAL is a multi-disciplinary open access archive for the deposit and dissemination of scientific research documents, whether they are published or not. The documents may come from teaching and research institutions in France or abroad, or from public or private research centers.
L'archive ouverte pluridisciplinaire HAL, est destinée au dépôt et à la diffusion de documents scientifiques de niveau recherche, publiés ou non, émanant des établissements d'enseignement et de recherche français ou étrangers, des laboratoires publics ou privés. 


\title{
Hybrid high-order methods for the acoustic wave equation in the time domain
}

\author{
Erik Burman* Omar Duran ${ }^{\dagger} \quad$ Alexandre Ern ${ }^{\ddagger}$
}

March 8, 2021

\begin{abstract}
We devise hybrid-high order (HHO) methods for the acoustic wave equation in the time domain. We first consider the second-order formulation in time. Using the Newmark scheme for the temporal discretization, we show that the resulting HHO-Newmark scheme is energy-conservative, and this scheme is also amenable to static condensation at each time step. We then consider the formulation of the acoustic wave equation as a firstorder system together with singly-diagonally implicit and explicit Runge-Kutta (SDIRK and ERK) schemes. HHO-SDIRK schemes are amenable to static condensation at each time step. For HHO-ERK schemes, the use of the mixed-order formulation, where the polynomial degree of the cell unknowns is one order higher than that of the face unknowns, is key to benefit from the explicit structure of the scheme. Numerical results on test cases with analytical solutions show that the methods can deliver optimal convergence rates for smooth solutions of order $\mathcal{O}\left(h^{k+1}\right)$ in the $H^{1}$-norm and of order $\mathcal{O}\left(h^{k+2}\right)$ in the $L^{2}$-norm. Moreover, test cases on wave propagation in heterogeneous media indicate the benefits of using high-order methods.
\end{abstract}

Mathematics Subjects Classification. 65M22, 65M12, 65M60, 35L05.

Keywords. Hybrid high-order methods, wave equation, Newmark scheme, Runge-Kutta scheme

\section{Introduction}

Hybrid high-order (HHO) methods were introduced in [26] for linear diffusion problems and in [25] for locking-free linear elasticity. For diffusion problems, these methods rely on hybrid unknowns which are polynomials of arbitrary order $k \geq 0$ on each mesh face, and on volume unknowns which are polynomials of order $k^{\prime} \in\{k-1, k, k+1\}, k^{\prime} \geq 0$, in each mesh cell. The cell unknowns can be eliminated locally by static condensation leading to a global transmission problem posed solely in terms of the face unknowns. HHO methods offer various assets (support of polyhedral meshes, local conservation principles, optimal convergence rates, and computational efficiency), and their devising hinges on two operators, both defined locally in each mesh cell: a gradient reconstruction operator and a stabilization operator. HHO methods have been bridged in [19] to hybridizable discontinuous Galerkin (HDG) methods [21] and nonconforming virtual element methods [5].

\footnotetext{
${ }^{*}$ Department of Mathematics, University College London, London, UK-WC1E 6BT, UK. E-mail address: e.burman@ucl.ac.uk

${ }^{\dagger}$ CERMICS, Ecole des Ponts, 77455 Marne la Vallée cedex 2, and INRIA Paris, 75589 Paris, France. E-mail address: omar.duran@enpc.fr

${ }^{\ddagger}$ CERMICS, Ecole des Ponts, 77455 Marne la Vallée cedex 2, and INRIA Paris, 75589 Paris, France. E-mail address: alexandre.ern@enpc.fr
} 
HHO methods have been extended to cover several applications in solid mechanics (e.g., nonlinear elasticity [9], hyperelasticity [1], and elastoplasticity [2]) and in fluid mechanics (e.g., Stokes [27], Navier-Stokes [8] and viscoplastic [14] flows). Moreover, HHO methods on unfitted meshes have been developed in [11], and the spectral approximation of elliptic operators by HHO methods has been analyzed in [13]. In contrast to the above, HHO methods have been less explored for time-dependent problems, and the main goal of the present work is to partly fill this gap by devising HHO methods for the approximation of wave propagation problems in the time domain. To motivate our work, we observe that the numerical simulation of wave propagation is relevant in many contexts, including acoustics, elastodynamics, and electromagnetics. Salient applications can be found in many areas, including aerospace, geophysics, civil engineering, telecommunications, and medicine for instance. In the present work, we focus on the acoustic wave equation. The extension to elastodynamic waves is relatively straightforward and is reported in [10], whereas the extension to electromagnetics could, e.g., be based on the ideas developed in [15] for magnetostatics.

The main results contained in this work can be described as follows. We first consider the acoustic wave equation in its primal form with a second-order time derivative. The HHO space semi-discretization relies on the method devised for elliptic problems, and the time discretization is based on the Newmark scheme. We show that the fully discrete problem enjoys an algebraic energy balance where the potential energy is evaluated using the HHO gradient reconstruction operator and also includes the HHO stabilization term. Recall that the Newmark scheme is an implicit, second-order accurate scheme that is unconditionally stable. The HHO-Newmark scheme is amenable to static condensation leading at each time step to a global linear system coupling only the face unknowns. We can also consider the leapfrog (or central finite difference) scheme to discretize the time derivative, which leads to an explicit, second-order accurate scheme which is conditionally stable and conserves exactly a modified energy. Unfortunately, the handling of the face unknowns still involves a sparse matrix coupling all the mesh faces, which hampers the benefit of using an explicit time-stepping scheme.

We next consider the acoustic wave equation in its mixed form as a first-order system. The space semi-discretization relates the velocity to the dual variable by means of the HHO gradient reconstruction operator, and we show that the stabilization term now acts as a dissipative contribution to the discrete energy balance. The temporal discretization can be achieved by either singly-diagonally implicit Runge-Kutta (SDIRK) and explicit Runge-Kutta (ERK) methods. One important observation is that it is necessary to consider the mixed-order case, where the cell unknowns are one polynomial order higher than the face unknowns (i.e., $k^{\prime}=k+1$ ), to actually benefit from the explicit nature of ERK schemes. Indeed, only in this situation, the submatrix representing the stabilization bilinear form for the face unknowns is block-diagonal. On the other hand, HHO-SDIRK schemes are amenable to static condensation. Moreover, inspired by the work [19] on elliptic problems, we bridge the present HHO methods to the HDG methods devised in the literature for acoustic wave propagation problems. Finally, our numerical results on test cases with smooth solutions show that the present methods can deliver $H^{1}$ and $L^{2}$-error estimates (say, at the final time) that converge optimally as $\mathcal{O}\left(h^{k+1}\right)$ and $\mathcal{O}\left(h^{k+2}\right)$, respectively, where $h$ is the mesh-size. These convergence rates are, at this stage, numerical observations, and we refer the reader to [10] for the error analysis. We also study the performance of the HHO-Newmark, SDIRK, and ERK methods to approximate wave propagation problems in heterogeneous media, and show in particular how the increase in the polynomial degree is beneficial to improve the accuracy and to temper energy dissipation. We also compare the computational efficiency of the explicit and implicit approaches to solve a two-dimensional heterogeneous wave propagation problem.

Let us put our work in perspective with the literature. The literature on finite element meth- 
ods for wave propagation problems is particularly rich. One of the main issues when considering high-order continuous finite element and mixed finite element settings combined with explicit time-stepping schemes is to devise suitably lumped mass matrices [7, 22, 23, 30]. In contrast, the use of discontinuous Galerkin (dG) methods naturally leads to block-diagonal mass matrices [33]. The $\mathrm{dG}$ discretization is often applied to the formulation of the wave equation as a first-order system [29, 38, 17, 28, 16], but the second-order formulation in time has been analyzed e.g., in [32] using the interior penalty $\mathrm{dG}$ method for the space semi-discretization. Notice in particular that $[17,16]$ specifically address the issue of preserving a discrete energy balance. Other relevant references for the approximation of wave equations in the time domain include, among others, $[3,4,6,42,45]$.

One drawback often invoked for dG methods in the context of implicit time-stepping schemes is their computational cost. Motivated in particular by this observation, HDG methods have been devised in $[40,39]$ for the wave equation formulated as a first-order system. The possibility of devising explicit HDG schemes has been uncovered in [43] by exploiting, as in the present work, the block-diagonal nature of the stabilization term acting on the face components. A computational comparison of explicit and implicit HDG schemes has been carried over in [35], indicating the better performance of explicit schemes despite their conditional stability. HDG methods combined with a Petrov-Galerkin discretization in time have been analyzed in [31]. Therein, the dissipative contribution of the stabilization term to the energy balance is pointed out, and a modification of the numerical flux trace is proposed to recover the exact balance of a modified energy including the stabilization term. HDG methods on the second-order formulation of the wave equation have been considered in $[41,20]$ to derive conservation properties. The findings of the present work on HHO methods are in line with previous work on HDG methods and shed further light on the connections between the two space discretization methods. In particular, exact energy conservation is achieved here using the HHO-Newmark scheme, and an explicit time-stepping implementation of HHO-ERK schemes is possible when using the formulation as a first-order setting and a mixed-order approximation of the two variables.

This paper is organized as follows. In Section 2, we present the model problem and recall the main ideas underlying the discretization of an elliptic problem by HHO methods. In Section 3, we describe the HHO discretization of the acoustics wave equation using its second-order formulation in time, and we do the same in Section 4 for the first-order formulation in time. We discuss the numerical results in Section 5. Finally, we draw some conclusions in Section 6.

\section{Continuous and discrete settings}

In this section, we present the two formulations of the acoustic wave equation and recall the main ideas of HHO methods to discretize the differential operator in space.

\subsection{Model problem and basic notation}

Let $\Omega$ be an open, bounded, connected subset of $\mathbb{R}^{d}, d \in\{1,2,3\}$, with a Lipschitz boundary $\Gamma$. For simplicity, we assume that $\Omega$ is a polyhedron. Let $J:=\left(0, T_{\mathrm{f}}\right)$ be the time interval with $T_{\mathrm{f}}>0$. We use standard notation for the Lebesgue and Sobolev spaces, as well as for the Bochner-Sobolev spaces in the space-time setting. Boldface notation is used for vectors and vector-valued fields. For a weight function $\phi \in L^{\infty}(\Omega)$ taking positive values uniformly bounded from below away from zero, we introduce the shorthand notation $\|v\|_{L^{2}(\phi ; \Omega)}:=\left\|\phi^{\frac{1}{2}} v\right\|_{L^{2}(\Omega)}$ for all $v \in L^{2}(\Omega)$, together with a similar notation for vector-valued fields in $\boldsymbol{L}^{2}(\Omega)$. 
The second-order formulation in time of the acoustic wave equation reads as follows:

$$
\frac{1}{\kappa} \partial_{t t} p-\nabla \cdot\left(\frac{1}{\rho} \nabla p\right)=f \quad \text { in } J \times \Omega,
$$

where $f\left[\frac{1}{\mathrm{~s}^{2}}\right]$ is the source term, $p[\mathrm{~Pa}]$ is the fluid pressure, $\kappa[\mathrm{Pa}]$ is the fluid bulk modulus, and $\rho$ $\left[\frac{\mathrm{kg}}{\mathrm{m}^{3}}\right]$ is the fluid density. For a derivation of the PDE (1) from the linearized Euler equations, we refer the reader to [23, Chap. 1, p. 3]. The second-order acoustic wave equation (1) is subjected to the initial conditions

$$
\left\{\begin{aligned}
p(\cdot, t=0) & =p_{0} \\
\partial_{t} p(\cdot, t=0) & =v_{0}
\end{aligned} \quad \text { in } \Omega,\right.
$$

and for simplicity we consider the homogeneous Dirichlet condition

$$
p=0 \quad \text { on } J \times \Gamma .
$$

We assume that the coefficients $\kappa$ and $\rho$ are piecewise constant on a partition of $\Omega$ into a finite collection of polyhedral subdomains, and that both coefficients take positive values. The speed of sound is defined as $c:=\sqrt{\frac{\kappa}{\rho}}$. We assume that $f \in L^{2}\left(J ; L^{2}(\Omega)\right), p_{0} \in H_{0}^{1}(\Omega)$, and $v_{0} \in L^{2}(\Omega)$. A reasonable functional setting to define the weak solution to (1)-(3) is $p \in L^{2}\left(J ; H_{0}^{1}(\Omega)\right.$ ), $\partial_{t} p \in L^{2}\left(J ; L^{2}(\Omega)\right)$, and $\partial_{t t} p \in L^{2}\left(J ; H^{-1}(\Omega)\right)$. Actually, our assumptions on the data imply that the weak solution is smoother, i.e., $p \in C^{0}\left(\bar{J} ; H_{0}^{1}(\Omega)\right) \cap C^{1}\left(\bar{J} ; L^{2}(\Omega)\right)$; see, e.g., [36, Chap. III, Thm. 8.1\&8.2]. Assuming that $p \in H^{2}\left(J ; L^{2}(\Omega)\right)$, we have for a.e. $t \in J$,

$$
\left(\partial_{t t} p(t), q\right)_{L^{2}\left(\frac{1}{\kappa} ; \Omega\right)}+b(p(t), q)=(f(t), q)_{L^{2}(\Omega)}, \quad \forall q \in H_{0}^{1}(\Omega),
$$

with the bilinear form $b: H_{0}^{1}(\Omega) \times H_{0}^{1}(\Omega) \rightarrow \mathbb{R}$ such that

$$
b(v, w):=(\nabla v, \nabla w)_{L^{2}\left(\frac{1}{\rho} ; \Omega\right)} .
$$

A crucial property of the acoustic wave equation is energy balance. The time-dependent energy functional is defined as follows for all $t \in \bar{J}$ :

$$
E(t):=\frac{1}{2}\left\|\partial_{t} p(t)\right\|_{L^{2}\left(\frac{1}{\kappa} ; \Omega\right)}^{2}+\frac{1}{2}\|\nabla p(t)\|_{L^{2}\left(\frac{1}{\rho} ; \Omega\right)}^{2} .
$$

Testing (4) against $q:=\partial_{t} p(t)$ implies that $\frac{d}{d t} E(t)=\left(f(t), \partial_{t} p(t)\right)_{L^{2}(\Omega)}$ for all $t \in J$. Integrating over $(0, t)$ then leads to the following well-known energy balance equation:

$$
E(t)=E(0)+\int_{0}^{t}\left(f(s), \partial_{t} p(s)\right)_{L^{2}(\Omega)} d s, \quad \forall t \in \bar{J},
$$

with $E(0)=\frac{1}{2}\left\|v_{0}\right\|_{L^{2}\left(\frac{1}{\kappa} ; \Omega\right)}^{2}+\frac{1}{2}\left\|\nabla p_{0}\right\|_{L^{2}\left(\frac{1}{\rho} ; \Omega\right)}^{2}$ owing to the initial condition (2). If the source term vanishes, (7) implies energy conservation: $E(t)=E(0)$ for all $t \in \bar{J}$.

A classical reformulation of the second-order PDE (1) is obtained by introducing two auxiliary variables, the scalar velocity $v:=\partial_{t} p\left[\frac{\mathrm{Pa}}{\mathrm{s}}\right]$ and the dual variable $\boldsymbol{\sigma}:=\frac{1}{\rho} \nabla p\left[\frac{\mathrm{m}}{\mathrm{s}^{2}}\right]$. This leads to the following coupled PDEs:

$$
\left\{\begin{aligned}
\rho \partial_{t} \boldsymbol{\sigma}-\nabla v & =\mathbf{0} \\
\frac{1}{\kappa} \partial_{t} v-\nabla \cdot \boldsymbol{\sigma} & =f
\end{aligned} \quad \text { in } J \times \Omega,\right.
$$


together with the initial conditions

$$
\boldsymbol{\sigma}(\cdot, t=0)=\frac{1}{\rho} \nabla p_{0}, \quad v(\cdot, t=0)=v_{0} \quad \text { in } \Omega,
$$

and the boundary condition

$$
v=0 \quad \text { on } J \times \Gamma .
$$

The above functional setting implies that $(\boldsymbol{\sigma}, v) \in C^{0}\left(\bar{J} ; \boldsymbol{L}^{2}(\Omega) \times L^{2}(\Omega)\right)$. Assuming that $v \in$ $H^{1}\left(J ; L^{2}(\Omega)\right) \cap L^{2}\left(J ; H_{0}^{1}(\Omega)\right)$ and $\boldsymbol{\sigma} \in H^{1}\left(J ; \boldsymbol{L}^{2}(\Omega)\right.$ ) (other functional settings are possible for the mixed formulation), we obtain

$$
\left\{\begin{aligned}
\left(\partial_{t} \boldsymbol{\sigma}(t), \boldsymbol{\tau}\right)_{L^{2}(\rho ; \Omega)}-(\nabla v(t), \boldsymbol{\tau})_{L^{2}(\Omega)} & =0, \\
\left(\partial_{t} v(t), w\right)_{L^{2}\left(\frac{1}{\kappa} ; \Omega\right)}+(\boldsymbol{\sigma}(t), \nabla w)_{L^{2}(\Omega)} & =(f(t), w)_{L^{2}(\Omega)},
\end{aligned}\right.
$$

for all $(\boldsymbol{\tau}, w) \in \boldsymbol{L}^{2}(\Omega) \times H_{0}^{1}(\Omega)$ and a.e. $t \in J$. In the first-order formulation, the time-dependent energy functional becomes for all $t \in \bar{J}$,

$$
E^{\mathrm{mix}}(t):=\frac{1}{2}\|v(t)\|_{L^{2}\left(\frac{1}{\kappa} ; \Omega\right)}^{2}+\frac{1}{2}\|\boldsymbol{\sigma}(t)\|_{\boldsymbol{L}^{2}(\rho ; \Omega)}^{2} .
$$

Clearly, $E^{\text {mix }}(t)=E(t)$, so that $E^{\text {mix }}(t)=E(0)+\int_{0}^{t}(f(s), v(s))_{L^{2}(\Omega)} d s$ for all $t \in \bar{J}$. The energy balance can also be directly deduced from (11) by testing the first equation against $\boldsymbol{\tau}:=\boldsymbol{\sigma}(t)$, the second against $w:=v(t)$, summing the result, and integrating in time.

\subsection{HHO discretization in space}

The goal of this section is to briefly outline the HHO discretization of the following steady diffusion problem:

$$
\text { Find } u \in H_{0}^{1}(\Omega) \text { s.t. } b(u, w)=(g, w)_{L^{2}(\Omega)} \forall w \in H_{0}^{1}(\Omega),
$$

with the source term $g \in L^{2}(\Omega)$ and the bilinear form $b$ defined in (5).

Let $\left(\mathcal{T}_{h}\right)_{h>0}$ be a sequence of meshes of $\Omega$ so that each mesh covers $\Omega$ exactly. We assume that each mesh $\mathcal{T}_{h}$ fits the partition of $\Omega$ into polyhedral subdomains so that both coefficients $\kappa$ and $\rho$ are piecewise constant on $\mathcal{T}_{h}$. For all $h>0, \mathcal{T}_{h}$ is composed of cells that can be polyhedral in $\mathbb{R}^{d}$ (with planar faces), and hanging nodes are possible. The mesh faces are collected in the set $\mathcal{F}_{h}$ which is split into $\mathcal{F}_{h}=\mathcal{F}_{h}^{\circ} \cup \mathcal{F}_{h}^{\partial}$, where $\mathcal{F}_{h}^{\circ}$ is the collection of the mesh interfaces and $\mathcal{F}_{h}^{\partial}$ is the collection of the boundary faces. A generic cell is denoted $T \in \mathcal{T}_{h}$, its diameter $h_{T}$, its unit outward normal $\boldsymbol{n}_{T}$, and the faces composing the boundary of $T$ are collected in the set $\mathcal{F}_{\partial T}$. The sequence $\left(\mathcal{T}_{h}\right)_{h>0}$ is assumed to be shape-regular in the sense of [25]. In a nutshell, the polyhedral mesh $\mathcal{T}_{h}$ admits for all $h>0$ a simplicial submesh $\mathcal{T}_{h}^{\prime}$ such that any cell (or face) of $\mathcal{T}_{h}^{\prime}$ is a subset of a exactly one cell (or at most one face) of $\mathcal{T}_{h}$, and there exists a shape-regularity parameter $\rho>0$ such that for all $h>0$, all $T \in \mathcal{T}_{h}$, and all $S \in \mathcal{T}_{h}^{\prime}$ such that $S \subset T$, we have $\rho h_{T} \leq h_{S} \leq \rho^{-1} r_{S}$, where $r_{S}$ and $h_{S}$ denote the inradius and the diameter of the simplex $S$. For simplicity, the reader can consider a shape-regular sequence of simplicial meshes without missing anything essential in what follows.

The discretization of (13) is realized by introducing discrete unknowns attached to the mesh cells and to the mesh faces. The homogeneous Dirichlet condition is enforced explicitly by zeroing out the discrete unknowns attached to the boundary faces. Let $k \geq 0$ be the polynomial degree used for the face unknowns and let $k^{\prime} \in\{k, k+1\}$ be the polynomial degree used for the cell 
unknowns. We say that the HHO discretization is of equal-order if $k^{\prime}=k$ and of mixed-order if $k^{\prime}=k+1$. The choice $k^{\prime}=k-1$ is also possible, but is not further discussed here since it essentially leads to the same developments as the equal-order choice $k^{\prime}=k$. Let us set

$$
\hat{V}_{h}:=V_{\mathcal{T}}^{k^{\prime}} \times V_{\mathcal{F}}^{k}, \quad V_{\mathcal{T}}^{k^{\prime}}:=\underset{T \in \mathcal{T}_{h}}{\chi} \mathbb{P}^{k^{\prime}}(T ; \mathbb{R}), \quad V_{\mathcal{F}}^{k}:=\underset{F \in \mathcal{F}_{h}}{\chi} \mathbb{P}^{k}(F ; \mathbb{R}),
$$

where $\mathbb{P}^{k^{\prime}}(T ; \mathbb{R})$ (resp., $\mathbb{P}^{k}(F ; \mathbb{R})$ ) consists of the restriction to $T$ (resp., $F$ ) of scalar-valued $d$ variate polynomials of degree at most $k^{\prime}$ (resp., $(d-1)$-variate polynomials of degree at most $k$ composed with any affine geometric mapping from the hyperplane supporting $F$ to $\mathbb{R}^{d-1}$ ). A generic element in $\hat{V}_{h}$ is denoted $\hat{v}_{h}:=\left(v_{\mathcal{T}}, v_{\mathcal{F}}\right)$, and we write $v_{T}$ (resp., $\left.v_{F}\right)$ for the component of $\hat{v}_{h}$ attached to a generic mesh cell $T \in \mathcal{T}_{h}$ (resp., face $F \in \mathcal{F}_{h}$ ). Let $\hat{v}_{h} \in \hat{V}_{h}$ and let $T \in \mathcal{T}_{h}$. The local components of $\hat{v}_{h}$ attached to the cell $T$ and its faces $F \in \mathcal{F}_{\partial T}$ are denoted

$$
\hat{v}_{T}:=\left(v_{T}, v_{\partial T}:=\left(v_{F}\right)_{F \in \mathcal{F}_{\partial T}}\right) \in \hat{V}_{T}:=V_{T}^{k^{\prime}} \times V_{\partial T}^{k},
$$

with $V_{T}^{k^{\prime}}:=\mathbb{P}^{k^{\prime}}(T ; \mathbb{R})$ and $V_{\partial T}^{k}:=\chi_{F \in \mathcal{F}_{\partial T}} \mathbb{P}^{k}(F ; \mathbb{R})$. To enforce the homogeneous Dirichlet condition, we consider the subspaces

$$
\left\{\begin{array}{l}
V_{\mathcal{F} 0}^{k}:=\left\{v_{\mathcal{F}} \in V_{\mathcal{F}}^{k} \mid v_{F}=0 \forall F \in \mathcal{F}_{h}^{\partial}\right\}, \\
\hat{V}_{h 0}:=V_{\mathcal{T}}^{k^{\prime}} \times V_{\mathcal{F} 0}^{k}=\left\{\hat{v}_{h} \in \hat{V}_{h} \mid v_{F}=0 \forall F \in \mathcal{F}_{h}^{\partial}\right\} .
\end{array}\right.
$$

Let $\Pi_{T}^{k^{\prime}}$ (resp., $\Pi_{\partial T}^{k}$ ) be the $L^{2}(T)$-orthogonal (resp., $L^{2}(\partial T)$-orthogonal) projection onto $V_{T}^{k^{\prime}}$ (resp., $V_{\partial T}^{k}$ ). A simple and natural way to approximate a given function $v \in H^{1}(\Omega)$ by a discrete pair $\hat{v}_{h} \in \hat{V}_{h}$ is to set $\hat{v}_{h}:=\hat{I}_{h}(v)$, where $\hat{I}_{h}: H^{1}(\Omega) \rightarrow \hat{V}_{h}$ is such that the local components of $\hat{I}_{h}(v)$ associated with a cell $T \in \mathcal{T}_{h}$ are $\hat{I}_{T}(v):=\left(\Pi_{T}^{k^{\prime}}(v), \Pi_{\partial T}^{k}(v)\right) \in \hat{V}_{T}$. Notice that this definition is meaningful since a function $v \in H^{1}(\Omega)$ does not jump across the mesh interfaces. In addition, we observe that $\hat{I}_{h}(v) \in \hat{V}_{h 0}$ whenever $v \in H_{0}^{1}(\Omega)$.

The HHO discretization is formulated locally by defining a gradient reconstruction and a stabilization operator in each mesh cell. The discrete problem is then assembled by summing the contributions of all the mesh cells. The gradient reconstruction operator $\boldsymbol{G}_{T}: \hat{V}_{T} \rightarrow \mathbb{P}^{k}\left(T ; \mathbb{R}^{d}\right)$ is such that for all $\hat{v}_{T} \in \hat{V}_{T}$, we have

$$
\left(\boldsymbol{G}_{T}\left(\hat{v}_{T}\right), \boldsymbol{q}\right)_{\boldsymbol{L}^{2}(T)}=-\left(v_{T}, \nabla \cdot \boldsymbol{q}\right)_{L^{2}(T)}+\left(v_{\partial T}, \boldsymbol{q} \cdot \boldsymbol{n}_{T}\right)_{L^{2}(\partial T)}, \quad \forall \boldsymbol{q} \in \mathbb{P}^{k}\left(T ; \mathbb{R}^{d}\right) .
$$

Notice that $\boldsymbol{G}_{T}\left(\hat{v}_{T}\right)$ can be evaluated componentwise by inverting the mass matrix associated with a basis of the scalar-valued polynomial space $\mathbb{P}^{k}(T ; \mathbb{R})$. An alternative to the gradient reconstruction operator is the potential reconstruction operator $R_{T}: \hat{V}_{T} \rightarrow \mathbb{P}^{k+1}(T ; \mathbb{R})$ such that for all $\hat{v}_{T} \in \hat{V}_{T}$, we have

$$
\left(\nabla R_{T}\left(\hat{v}_{T}\right), \nabla q\right)_{L^{2}(T)}=-\left(v_{T}, \Delta q\right)_{L^{2}(T)}+\left(v_{\partial T}, \nabla q \cdot \boldsymbol{n}_{T}\right)_{L^{2}(\partial T)}, \quad \forall q \in \mathbb{P}^{k+1}(T ; \mathbb{R}),
$$

together with the mean-value condition $\left(R_{T}\left(\hat{v}_{T}\right)-v_{T}, 1\right)_{L^{2}(T)}=0$. Notice that $R_{T}\left(\hat{v}_{T}\right)$ can be evaluated componentwise by inverting the stiffness matrix associated with a basis of the scalarvalued polynomial space $\mathbb{P}^{k+1}(T ; \mathbb{R}) / \mathbb{R}$. Furthermore, the role of the stabilization operator is to enforce weakly the matching of the trace of the cell unknown with the local face unknowns. For all $\hat{v}_{T} \in \hat{V}_{T}$, let us set $\delta_{\partial T}\left(\hat{v}_{T}\right):=v_{T \mid \partial T}-v_{\partial T}$. Then, in the equal-order case $\left(k^{\prime}=k\right)$, we set

$$
S_{\partial T}\left(\hat{v}_{T}\right):=\Pi_{\partial T}^{k}\left(\delta_{\partial T}\left(\hat{v}_{T}\right)+\left(\left(I-\Pi_{T}^{k}\right) R_{T}\left(0, \delta_{\partial T}\left(\hat{v}_{T}\right)\right)\right)_{\mid \partial T}\right),
$$

and in the mixed-order case $\left(k^{\prime}=k+1\right)$, we set

$$
S_{\partial T}\left(\hat{v}_{T}\right):=\Pi_{\partial T}^{k}\left(\delta_{\partial T}\left(\hat{v}_{T}\right)\right) .
$$


Finally, we define the local discrete bilinear form $b_{T}: \hat{V}_{T} \times \hat{V}_{T} \rightarrow \mathbb{R}$ such that

$$
b_{T}\left(\hat{v}_{T}, \hat{w}_{T}\right):=\left(\boldsymbol{G}_{T}\left(\hat{v}_{T}\right), \boldsymbol{G}_{T}\left(\hat{w}_{T}\right)\right)_{\boldsymbol{L}^{2}\left(\frac{1}{\rho} ; T\right)}+\tau_{\partial T}\left(S_{\partial T}\left(\hat{v}_{T}\right), S_{\partial T}\left(\hat{w}_{T}\right)\right)_{L^{2}(\partial T)},
$$

with the weight $\tau_{\partial T}:=\left(\rho_{T} h_{T}\right)^{-1}$. Defining the global discrete bilinear form $b_{h}: \hat{V}_{h} \times \hat{V}_{h} \rightarrow \mathbb{R}$ such that $b_{h}\left(\hat{v}_{h}, \hat{w}_{h}\right):=\sum_{T \in \mathcal{T}_{h}} b_{T}\left(\hat{v}_{T}, \hat{w}_{T}\right)$, the HHO discretization of the model problem (13) is as follows:

$$
\text { Find } \hat{u}_{h} \in \hat{V}_{h 0} \text { s.t. } b_{h}\left(\hat{u}_{h}, \hat{w}_{h}\right)=\left(g, w_{\mathcal{T}}\right)_{L^{2}(\Omega)} \forall \hat{w}_{h} \in \hat{V}_{h 0} \text {. }
$$

Notice that only the cell component of the discrete test function $\hat{w}_{h}:=\left(w_{\mathcal{T}}, w_{\mathcal{F}}\right)$ is used on the right-hand side. It is convenient to define the global gradient reconstruction operator $\boldsymbol{G}_{\mathcal{T}}: \hat{V}_{h} \rightarrow$ $\boldsymbol{W}_{\mathcal{T}}:=\times_{T \in \mathcal{T}_{h}} \mathbb{P}^{k}\left(T ; \mathbb{R}^{d}\right)$ such that $\left(\boldsymbol{G}_{\mathcal{T}}\left(\hat{v}_{h}\right)\right)_{\mid T}:=\boldsymbol{G}_{T}\left(\hat{v}_{T}\right)$ for all $T \in \mathcal{T}_{h}$ and all $\hat{v}_{h} \in \hat{V}_{h}$, and to set $s_{h}\left(\hat{v}_{h}, \hat{w}_{h}\right):=\sum_{T \in \mathcal{T}_{h}} \tau_{\partial T}\left(S_{\partial T}\left(\hat{v}_{T}\right), S_{\partial T}\left(\hat{w}_{T}\right)\right)_{L^{2}(\partial T)}$. Then we have

$$
b_{h}\left(\hat{v}_{h}, \hat{w}_{h}\right)=\left(\boldsymbol{G}_{\mathcal{T}}\left(\hat{v}_{h}\right), \boldsymbol{G}_{\mathcal{T}}\left(\hat{w}_{h}\right)\right)_{\boldsymbol{L}^{2}\left(\frac{1}{\rho} ; \Omega\right)}+s_{h}\left(\hat{v}_{h}, \hat{w}_{h}\right) .
$$

The cellwise assembly of the global discrete bilinear form $b_{h}$ makes the treatment of heterogeneous material properties very natural, as in the finite element method, since one only manipulates terms associated with a given mesh cell in which the material property is well-defined.

A direct verification shows that the map $\|\cdot\|_{\hat{V}_{h}}: \hat{V}_{h} \rightarrow \mathbb{R}$ such that

$$
\left\|\hat{v}_{h}\right\|_{\hat{V}_{h}}^{2}:=\sum_{T \in \mathcal{T}_{h}}\left(\left\|\nabla v_{T}\right\|_{L^{2}\left(\frac{1}{\rho} ; T\right)}^{2}+\tau_{\partial T}\left\|v_{\partial T}-v_{T}\right\|_{L^{2}(\partial T)}^{2}\right), \quad \forall \hat{v}_{h} \in \hat{V}_{h},
$$

defines a seminorm on $\hat{V}_{h}$ and a norm on $\hat{V}_{h 0}$. Additionally, we have the following important stability result (see $[26,25])$.

Lemma 2.1 (Stability). There are $0<\alpha \leq \varpi<\infty$ such that for all $\hat{v}_{h} \in \hat{V}_{h 0}$ and all $h>0$,

$$
\alpha\left\|\hat{v}_{h}\right\|_{\hat{V}_{h}}^{2} \leq\left\|\boldsymbol{G}_{\mathcal{T}}\left(\hat{v}_{h}\right)\right\|_{L^{2}\left(\frac{1}{\rho} ; \Omega\right)}^{2}+\left|\hat{v}_{h}\right|_{S}^{2} \leq \varpi\left\|\hat{v}_{h}\right\|_{\hat{V}_{h}}^{2},
$$

with the seminorm $\left|\hat{v}_{h}\right|_{S}^{2}:=s_{h}\left(\hat{v}_{h}, \hat{v}_{h}\right)$.

Finally, the following approximation result is the key argument to derive optimal $H^{1}$-error estimates (see again $[26,25]$ ).

Lemma 2.2 (Approximation). There is $C$ s.t. for all $h>0$, all $T \in \mathcal{T}_{h}$, all $l \in\{1, \ldots, k+1\}$, and all $v \in H^{l}(T)$, we have

$$
\left\|\nabla v-\boldsymbol{G}_{T}\left(\hat{I}_{T}(v)\right)\right\|_{\boldsymbol{L}^{2}(T)}+\tau_{\partial T}^{\frac{1}{2}}\left\|S_{\partial T}\left(\hat{I}_{T}(v)\right)\right\|_{L^{2}(\partial T)} \leq C h_{T}^{l}|v|_{H^{l+1}(T)} .
$$

The above right-hand side converges optimally as $\mathcal{O}\left(h_{T}^{k+1}\right)$ whenever $l=k+1$.

Remark 2.3 (Reconstruction). The potential reconstruction operator $R_{T}$ is not needed in the mixed-order case, and in the equal-order case, it is only used to evaluate the local stabilization operator. Alternatively, one can also consider $\nabla R_{T}$ as a gradient reconstruction operator, leading to the modified bilinear form

$$
\tilde{b}_{T}\left(\hat{v}_{T}, \hat{w}_{T}\right):=\left(\nabla R_{T}\left(\hat{v}_{T}\right), \nabla R_{T}\left(\hat{w}_{T}\right)\right)_{L^{2}\left(\frac{1}{\rho} ; T\right)}+\tau_{\partial T}\left(S_{\partial T}\left(\hat{v}_{T}\right), S_{\partial T}\left(\hat{w}_{T}\right)\right)_{L^{2}(\partial T)} .
$$

This choice also leads to optimal error estimates. Using the gradient reconstruction operator $\boldsymbol{G}_{T}$ is preferable in the context of nonlinear problems [9, 24, 1, 2]. 


\section{HHO discretization for the second-order wave equation}

In this section, we present the space semi-discrete and the fully discrete HHO schemes for the second-order wave equation. The temporal discretization is performed by using Newmark schemes and variants. In the rest of the manuscript, we assume that the initial velocity $v_{0}$ is in $H_{0}^{1}(\Omega)$ (recall that the initial pressure $p_{0}$ is in $H_{0}^{1}(\Omega)$ by assumption). We also assume that $f \in C^{0}\left(\bar{J} ; L^{2}(\Omega)\right)$ to consider pointwise values in time of the source term when writing the time-stepping schemes.

\subsection{Space semi-discrete scheme}

The space semi-discrete HHO scheme for the second-order wave equation consists of finding $\hat{p}_{h}:=\left(p_{\mathcal{T}}, p_{\mathcal{F}}\right) \in C^{2}\left(\bar{J} ; \hat{V}_{h 0}\right)$ such that for all $t \in \bar{J}$,

$$
\left(\partial_{t t} p_{\mathcal{T}}(t), q_{\mathcal{T}}\right)_{L^{2}\left(\frac{1}{\kappa} ; \Omega\right)}+b_{h}\left(\hat{p}_{h}(t), \hat{q}_{h}\right)=\left(f(t), q_{\mathcal{T}}\right)_{L^{2}(\Omega)},
$$

for all $\hat{q}_{h}:=\left(q_{\mathcal{T}}, q_{\mathcal{F}}\right) \in \hat{V}_{h 0}$. The initial conditions for $(27)$ only concern $p_{\mathcal{T}}$ and are as follows:

$$
\left\{\begin{aligned}
p_{\mathcal{T}}(\cdot, t=0) & =\Pi_{\mathcal{T}}^{k^{\prime}}\left(p_{0}\right), \\
\partial_{t} p_{\mathcal{T}}(\cdot, t=0) & =\Pi_{\mathcal{T}}^{k^{\prime}}\left(v_{0}\right)
\end{aligned}\right.
$$

where $\Pi_{\mathcal{T}}^{k^{\prime}}$ is the global $\boldsymbol{L}^{2}$-orthogonal projection onto $V_{\mathcal{T}}^{k^{\prime}}$. The boundary condition is encoded in the fact that $\hat{p}_{h}(t) \in \hat{V}_{h 0}$ for all $t \in \bar{J}$.

Lemma 3.1 (Space semi-discrete energy balance). Let $\hat{p}_{h} \in C^{2}\left(\bar{J} ; \hat{V}_{h 0}\right)$ solve (27)-(28) and define the time-dependent space semi-discrete energy functional as

$$
\hat{E}(t):=\frac{1}{2}\left\|\partial_{t} p_{\mathcal{T}}(t)\right\|_{L^{2}\left(\frac{1}{\kappa} ; \Omega\right)}^{2}+\frac{1}{2}\left\|\boldsymbol{G}_{\mathcal{T}}\left(\hat{p}_{h}(t)\right)\right\|_{\boldsymbol{L}^{2}\left(\frac{1}{\rho} ; \Omega\right)}^{2}+\frac{1}{2}\left|\hat{p}_{h}(t)\right|_{S}^{2} .
$$

Then the following energy balance equation holds true for all $t \in J$ :

$$
\hat{E}(t)=\hat{E}(0)+\int_{0}^{t}\left(f(s), \partial_{t} p_{\mathcal{T}}(s)\right)_{L^{2}(\Omega)} d s .
$$

Proof. The proof is similar to the continuous case. One considers the test function $\hat{q}_{h}:=\partial_{t} \hat{p}_{h}(t)$ in (27) for all $t \in \bar{J}$, and since the discrete bilinear form $b_{h}$ is symmetric, this leads to

$$
\frac{d}{d t}\left(\frac{1}{2}\left\|\partial_{t} p_{\mathcal{T}}(t)\right\|_{L^{2}\left(\frac{1}{\kappa} ; \Omega\right)}^{2}+\frac{1}{2} b_{h}\left(\hat{p}_{h}(t), \hat{p}_{h}(t)\right)\right)=\left(f(t), \partial_{t} p_{\mathcal{T}}(t)\right)_{L^{2}(\Omega)} .
$$

Since $b_{h}\left(\hat{q}_{h}, \hat{q}_{h}\right)=\left\|\boldsymbol{G}_{\mathcal{T}}\left(\hat{q}_{h}\right)\right\|_{\boldsymbol{L}^{2}\left(\frac{1}{\rho} ; \Omega\right)}^{2}+\left|\hat{q}_{h}\right|_{S}^{2}$ for all $\hat{q}_{h} \in \hat{V}_{h 0}$, integrating the above identity in time over $(0, t)$ for all $t \in J$ leads to $(30)$.

Let $N_{\mathcal{T}}^{k^{\prime}}:=\operatorname{dim}\left(V_{\mathcal{T}}^{k^{\prime}}\right)$ and $N_{\mathcal{F}}^{k}:=\operatorname{dim}\left(V_{\mathcal{F} 0}^{k}\right)$. Let $\left(\mathrm{P}_{\mathcal{T}}(t), \mathrm{P}_{\mathcal{F}}(t)\right) \in \mathbb{R}^{N_{\mathcal{T}}^{k^{\prime}} \times N_{\mathcal{F}}^{k}}$ be the (timedependent) component vectors of the space semi-discrete solution $\hat{p}_{h}(t):=\left(p_{\mathcal{T}}(t), p_{\mathcal{F}}(t)\right) \in \hat{V}_{h 0}$ once bases $\left\{\varphi_{i}\right\}_{1 \leq i \leq N_{\mathcal{T}}^{k^{\prime}}}$ and $\left\{\psi_{j}\right\}_{1 \leq j \leq N_{\mathcal{F}}^{k}}$ for $V_{\mathcal{T}}^{k^{\prime}}$ and $V_{\mathcal{F} 0}^{k}$, respectively, have been chosen. Let $\mathrm{F}_{\mathcal{T}}(t) \in \mathbb{R}^{N_{\mathcal{T}}^{k^{\prime}}}$ have components given by $\mathrm{F}_{i}(t):=\left(f(t), \varphi_{i}\right)_{L^{2}(\Omega)}$ for all $t \in \bar{J}$ and all $1 \leq i \leq N_{\mathcal{T}}^{k^{\prime}}$. The algebraic realization of (27) is as follows: For all $t \in \bar{J}$,

$$
\left[\begin{array}{cc}
\mathrm{M}_{\mathcal{T} \mathcal{T}} & 0 \\
0 & 0
\end{array}\right]\left[\begin{array}{c}
\partial_{t t} \mathrm{P}_{\mathcal{T}}(t) \\
\bullet
\end{array}\right]+\left[\begin{array}{ll}
\mathrm{K}_{\mathcal{T} \mathcal{T}} & \mathrm{K}_{\mathcal{T} \mathcal{F}} \\
\mathrm{K}_{\mathcal{F} \mathcal{T}} & \mathrm{K}_{\mathcal{F F}}
\end{array}\right]\left[\begin{array}{l}
\mathrm{P}_{\mathcal{T}}(t) \\
\mathrm{P}_{\mathcal{F}}(t)
\end{array}\right]=\left[\begin{array}{c}
\mathrm{F}_{\mathcal{T}}(t) \\
0
\end{array}\right]
$$


with the mass matrix $\mathbf{M}_{\mathcal{T} \mathcal{T}}$ associated with the inner product in $L^{2}\left(\frac{1}{\kappa} ; \Omega\right)$ and the cell basis functions, and the symmetric positive-definite stiffness matrix with blocks $\mathrm{K}_{\mathcal{T} \mathcal{T}}, \mathrm{K}_{\mathcal{T} \mathcal{F}}, \mathrm{K}_{\mathcal{F} \mathcal{T}}$, $\mathrm{K}_{\mathcal{F F}}$ associated with the bilinear form $b_{h}$ and the cell and face basis functions. The bullet stands for $\partial_{t t} \mathrm{P}_{\mathcal{F}}(t)$ which is irrelevant owing to the structure of the mass matrix. An important observation is that the matrices $\mathbf{M}_{\mathcal{T} \mathcal{T}}$ and $\mathrm{K}_{\mathcal{T} \mathcal{T}}$ are block-diagonal. This is not the case for the matrix $\mathrm{K}_{\mathcal{F F}}$ since the components attached to faces belonging to the same cell are coupled together.

Remark 3.2 (Evolution problem with Schur complement). We notice that the face unknowns can be eliminated from (31). Indeed, defining the Schur complement $\check{\mathrm{K}}_{\mathcal{T} \mathcal{T}}:=\mathrm{K}_{\mathcal{T} \mathcal{T}}-\mathrm{K}_{\mathcal{T} \mathcal{F}} \mathrm{K}_{\mathcal{F} F}^{-1} \mathrm{~K}_{\mathcal{F} \mathcal{T}}$, which is a symmetric positive-definite matrix, (31) implies that for all $t \in \bar{J}$,

$$
\mathrm{M}_{\mathcal{T} \mathcal{T}} \partial_{t t} \mathrm{P}_{\mathcal{T}}(t)+\check{\mathrm{K}}_{\mathcal{T} \mathcal{T}} \mathrm{P}_{\mathcal{T}}(t)=\mathrm{F}_{\mathcal{T}}(t)
$$

The same reasoning can be applied to (27). Let us define the face recovery operator $Z_{\mathcal{F}}: V_{\mathcal{T}}^{k^{\prime}} \rightarrow$ $V_{\mathcal{F} 0}^{k}$ such that for all $q_{\mathcal{T}} \in V_{\mathcal{T}}^{k^{\prime}}, Z_{\mathcal{F}}\left(q_{\mathcal{T}}\right)$ is uniquely defined in $V_{\mathcal{F} 0}^{k}$ by solving the following well-posed problem:

$$
b_{h}\left(\left(q_{\mathcal{T}}, Z_{\mathcal{F}}\left(q_{\mathcal{T}}\right)\right),\left(0, r_{\mathcal{F}}\right)\right)=0 \quad \forall r_{\mathcal{F}} \in V_{\mathcal{F} 0}^{k} .
$$

(Notice that $Z_{\mathcal{F}}\left(q_{\mathcal{T}}\right)$ is the unique minimizer in $V_{\mathcal{F} 0}^{k}$ of the discrete energy functional $r_{\mathcal{F}} \mapsto$ $\frac{1}{2} b_{h}\left(\left(q_{\mathcal{T}}, r_{\mathcal{F}}\right),\left(q_{\mathcal{T}}, r_{\mathcal{F}}\right)\right)$.) Then $\hat{p}_{h} \in C^{2}\left(\bar{J} ; \hat{V}_{h 0}\right)$ solves $(27)$ if and only if $p_{\mathcal{F}}(t)=Z_{\mathcal{F}}\left(p_{\mathcal{T}}(t)\right)$ for all $t \in \bar{J}$ and $p_{\mathcal{T}} \in C^{2}\left(\bar{J} ; V_{\mathcal{T}}^{k^{\prime}}\right)$ solves the following reduced problem:

$$
\left(\partial_{t t} p_{\mathcal{T}}(t), q_{\mathcal{T}}\right)_{L^{2}\left(\frac{1}{\kappa} ; \Omega\right)}+b_{\mathcal{T}}\left(p_{\mathcal{T}}(t), q_{\mathcal{T}}\right)=\left(f(t), q_{\mathcal{T}}\right)_{L^{2}(\Omega)} \quad \forall q_{\mathcal{T}} \in V_{\mathcal{T}}^{k^{\prime}},
$$

where $b_{\mathcal{T}}\left(q_{\mathcal{T}}, r_{\mathcal{T}}\right):=b_{h}\left(\left(q_{\mathcal{T}}, Z_{\mathcal{F}}\left(q_{\mathcal{T}}\right)\right),\left(r_{\mathcal{T}}, Z_{\mathcal{F}}\left(r_{\mathcal{T}}\right)\right)\right)$ for all $q_{\mathcal{T}}, r_{\mathcal{T}} \in V_{\mathcal{T}}^{k^{\prime}}$

\section{$3.2 \quad$ Fully discrete scheme}

Let $\left(t^{n}\right)_{0 \leq n \leq N}$ be the discrete time nodes with $t^{0}:=0$ and $t^{N}:=T_{\mathrm{f}}$. We consider a fixed time step $\Delta t:=\frac{T_{\mathrm{f}}}{N}$. Let $\hat{p}_{h}^{n}:=\left(p_{\mathcal{T}}^{n}, p_{\mathcal{F}}^{n}\right) \in \hat{V}_{h 0}$ denote the temporal approximation of $\hat{p}_{h}\left(t^{n}\right)$. The corresponding component vector is denoted $\mathrm{P}^{n}:=\left(\mathrm{P}_{\mathcal{T}}^{n}, \mathrm{P}_{\mathcal{F}}^{n}\right) \in \mathbb{R}^{N_{\mathcal{T}}^{k^{\prime}} \times N_{\mathcal{F}}^{k}}$. Let us also set $\mathrm{F}^{n}:=\mathrm{F}\left(t^{n}\right)$ for all $n \in\{1, \ldots, N\}$.

A classical scheme for the temporal discretization of (27) is the leapfrog (or central finite difference) scheme. In the present setting, the HHO-leapfrog scheme consists of solving for all $n \in\{1, \ldots, N-1\}$,

$$
\frac{1}{\Delta t^{2}}\left(p_{\mathcal{T}}^{n+1}-2 p_{\mathcal{T}}^{n}+p_{\mathcal{T}}^{n-1}, q_{\mathcal{T}}\right)_{L^{2}\left(\frac{1}{\kappa} ; \Omega\right)}+b_{h}\left(\hat{p}_{h}^{n}, \hat{q}_{h}\right)=\left(f\left(t^{n}\right), q_{\mathcal{T}}\right)_{L^{2}(\Omega)},
$$

for all $\hat{q}_{h}:=\left(q_{\mathcal{T}}, q_{\mathcal{F}}\right) \in \hat{V}_{h 0}$, where $p_{\mathcal{T}}^{n}, p_{\mathcal{T}}^{n-1}$ are determined from the previous steps or the initial condition. We observe that (32) delivers an equation for $p_{\mathcal{T}}^{n+1}$ (when testing with $q_{\mathcal{T}}$ ) and one for $p_{\mathcal{F}}^{n}$ (when testing with $q_{\mathcal{F}}$ ). This structure becomes more apparent if one considers the algebraic form of (32) which reads

$$
\left[\begin{array}{cc}
\mathrm{M}_{\mathcal{T} \mathcal{T}} & 0 \\
0 & 0
\end{array}\right]\left[\begin{array}{c}
\mathrm{P}_{\mathcal{T}}^{n+1} \\
\bullet
\end{array}\right]+\Delta t^{2}\left[\begin{array}{ll}
\mathrm{K}_{\mathcal{T} \mathcal{T}} & \mathrm{K}_{\mathcal{T F}} \\
\mathrm{K}_{\mathcal{F} \mathcal{T}} & \mathrm{K}_{\mathcal{F} \mathcal{F}}
\end{array}\right]\left[\begin{array}{c}
\mathrm{P}_{\mathcal{T}}^{n} \\
\mathrm{P}_{\mathcal{F}}^{n}
\end{array}\right]=\left[\begin{array}{c}
\mathrm{G}_{\mathcal{T}}^{n} \\
0
\end{array}\right]
$$

with $\mathrm{G}_{\mathcal{T}}^{n}:=\Delta t^{2} \mathrm{~F}_{\mathcal{T}}^{n}+\mathrm{M}_{\mathcal{T} \mathcal{T}}\left(2 \mathrm{P}_{\mathcal{T}}^{n}-\mathrm{P}_{\mathcal{T}}^{n-1}\right)$. The bullet stands for $\mathrm{P}_{\mathcal{F}}^{n+1}$ which is irrelevant owing to the structure of the mass matrix. In other words, we must perform sequentially the following two substeps where $\mathrm{P}_{\mathcal{T}}^{n-1}, \mathrm{P}_{\mathcal{T}}^{n} \in \mathbb{R}^{N_{\mathcal{T}}^{k^{\prime}}}$ are known from the previous time step or the initial condition: 
1. Find $\mathrm{P}_{\mathcal{F}}^{n} \in \mathbb{R}^{N_{\mathcal{F}}^{k}}$ such that $\mathrm{K}_{\mathcal{F} \mathcal{F}} \mathrm{P}_{\mathcal{F}}^{n}=-\mathrm{K}_{\mathcal{F} \mathcal{T}} \mathrm{P}_{\mathcal{T}}^{n}$.

2. Find $\mathrm{P}_{\mathcal{T}}^{n+1} \in \mathbb{R}^{N_{\mathcal{T}}^{k^{\prime}}}$ such that $\mathrm{M}_{\mathcal{T} \mathcal{T}} \mathrm{P}_{\mathcal{T}}^{n+1}=\mathrm{G}_{\mathcal{T}}^{n}-\Delta t^{2}\left(\mathrm{~K}_{\mathcal{T} \mathcal{T}} \mathrm{P}_{\mathcal{T}}^{n}+\mathrm{K}_{\mathcal{T} \mathcal{F}} \mathrm{P}_{\mathcal{F}}^{n}\right)$.

The first substep entails dealing with a sparse symmetric positive-definite matrix coupling all the unknowns attached to the mesh faces. Instead, the matrix involved in the second substep is easy to invert since it is block-diagonal. The leapfrog scheme is second-order accurate in time and conditionally stable. It is in general considered to be an explicit scheme, but its realization in the present context requires the inversion of the sparse matrix $\mathrm{K}_{\mathcal{F} \mathcal{F}}$ which makes the scheme less attractive than the implicit schemes that we now consider.

Another classical scheme for the temporal discretization of (27) is the Newmark scheme with real parameters $\beta$ and $\gamma$. This scheme is second-order accurate in time and unconditionally stable if $\frac{1}{2} \leq \gamma \leq 2 \beta$ (the classical choice for this situation is $\gamma=\frac{1}{2}$ and $\beta=\frac{1}{4}$ ) and conditionally stable if $\frac{1}{2} \leq \gamma$ and $2 \beta<\gamma$. The leapfrog scheme, which falls into the second category, corresponds to the choice $\gamma=\frac{1}{2}$ and $\beta=0$. The Newmark scheme is implicit as soon as $\beta>0$. The usual way of writing the Newmark scheme is to consider an approximation for the pressure, its velocity, and its acceleration at each time node, say $\hat{p}_{h}^{n}, \hat{v}_{h}^{n}, \hat{a}_{h}^{n} \in \hat{V}_{h 0}$. In the present setting, all of these quantities are hybrid variables. The scheme is initialized by setting $\hat{p}_{h}^{0}:=\hat{I}_{h}\left(p_{0}\right), \hat{v}_{h}^{0}:=\hat{I}_{h}\left(v_{0}\right)$, and the initial acceleration $\hat{a}_{h}^{0}:=\left(a_{\mathcal{T}}^{0}, a_{\mathcal{F}}^{0}\right) \in \hat{V}_{h 0}$ is defined by solving $\left(a_{\mathcal{T}}^{0}, q_{\mathcal{T}}\right)_{L^{2}\left(\frac{1}{\kappa} ; \Omega\right)}+b_{h}\left(\hat{p}_{h}^{0},\left(q_{\mathcal{T}}, 0\right)\right)=$ $\left(f\left(t^{0}\right), q_{\mathcal{T}}\right)_{L^{2}(\Omega)}$ for all $q_{\mathcal{T}} \in V_{\mathcal{T}}^{k^{\prime}}$, and $b_{h}\left(\hat{a}_{h}^{0},\left(0, q_{\mathcal{F}}\right)\right)=0$ for all $q_{\mathcal{F}} \in V_{\mathcal{F} 0}^{k}$. Then, given $\hat{p}_{h}^{n}, \hat{v}_{h}^{n}, \hat{a}_{h}^{n}$ from the previous time step or the initial condition, we obtain the following HHO-Newmark scheme: For all $n \in\{0, \ldots, N-1\}$,

$$
\left\{\begin{array}{l}
\left(a_{\mathcal{T}}^{n+1}, q_{\mathcal{T}}\right)_{L^{2}\left(\frac{1}{\kappa} ; \Omega\right)}+b_{h}\left(\hat{p}_{h}^{n+1}, \hat{q}_{h}\right)=\left(f\left(t^{n+1}\right), q_{\mathcal{T}}\right)_{L^{2}(\Omega)} \quad \forall \hat{q}_{h} \in \hat{V}_{h 0}, \\
\left.\hat{p}_{h}^{n+1}=\hat{p}_{h}^{n}+\Delta t \hat{v}_{h}^{n}+\frac{1}{2} \Delta t^{2}\left((1-2 \beta) \hat{a}_{h}^{n}+2 \beta \hat{a}_{h}^{n+1}\right)\right) \\
\left.\hat{v}_{h}^{n+1}=\hat{v}_{h}^{n}+\Delta t\left((1-\gamma) \hat{a}_{h}^{n}+\gamma \hat{a}_{h}^{n+1}\right)\right)
\end{array}\right.
$$

In practice, the implementation of (34) relies on the following three steps:

1. Predictor step: Set

$$
\left\{\begin{array}{l}
\hat{p}_{h}^{* n}:=\hat{p}_{h}^{n}+\Delta t \hat{v}_{h}^{n}+\frac{1}{2} \Delta t^{2}(1-2 \beta) \hat{a}_{h}^{n} \\
\hat{v}_{h}^{* n}:=\hat{v}_{h}^{n}+\Delta t(1-\gamma) \hat{a}_{h}^{n}
\end{array}\right.
$$

2. Linear solve to find the acceleration $\hat{a}_{h}^{n+1} \in \hat{V}_{h 0}$ such that for all $\hat{q}_{h} \in \hat{V}_{h 0}$,

$$
\left(a_{\mathcal{T}}^{n+1}, q_{\mathcal{T}}\right)_{L^{2}\left(\frac{1}{\kappa} ; \Omega\right)}+\beta \Delta t^{2} b_{h}\left(\hat{a}_{h}^{n+1}, \hat{q}_{h}\right)=\left(f\left(t^{n+1}\right), q_{\mathcal{T}}\right)_{L^{2}(\Omega)}-b_{h}\left(\hat{p}_{h}^{* n}, \hat{q}_{h}\right) .
$$

3. Corrector step: Set

$$
\left\{\begin{array}{l}
\hat{p}_{h}^{n+1}:=\hat{p}_{h}^{* n}+\beta \Delta t^{2} \hat{a}_{h}^{n+1}, \\
\hat{v}_{h}^{n+1}:=\hat{v}_{h}^{* n}+\gamma \Delta t \hat{a}_{h}^{n+1} .
\end{array}\right.
$$

The algebraic realization of the predictor and corrector steps is straightforward, and that of the second step amounts to finding $\left(\mathrm{A}_{\mathcal{T}}^{n+1}, \mathrm{~A}_{\mathcal{F}}^{n+1}\right) \in \mathbb{R}^{N_{\mathcal{T}}^{k^{\prime}} \times N_{\mathcal{F}}^{k}}$ such that

$$
\left(\left[\begin{array}{cc}
\mathrm{M}_{\mathcal{T} \mathcal{T}} & 0 \\
0 & 0
\end{array}\right]+\beta \Delta t^{2}\left[\begin{array}{ll}
\mathrm{K}_{\mathcal{T} \mathcal{T}} & \mathrm{K}_{\mathcal{T} \mathcal{F}} \\
\mathrm{K}_{\mathcal{F} \mathcal{T}} & \mathrm{K}_{\mathcal{F F}}
\end{array}\right]\right)\left[\begin{array}{l}
\mathrm{A}_{\mathcal{T}}^{n+1} \\
\mathrm{~A}_{\mathcal{F}}^{n+1}
\end{array}\right]=\left[\begin{array}{l}
\mathrm{B}_{\mathcal{T}}^{n+1} \\
\mathrm{~B}_{\mathcal{F}}^{n+1}
\end{array}\right]
$$

with $\mathrm{B}_{\mathcal{T}}^{n+1}:=\mathrm{F}_{\mathcal{T}}^{n+1}-\left(\mathrm{K}_{\mathcal{T} \mathcal{T}} \mathrm{P}_{\mathcal{T}}^{* n}+\mathrm{K}_{\mathcal{T} \mathcal{F}} \mathrm{P}_{\mathcal{F}}^{* n}\right), \mathrm{B}_{\mathcal{F}}^{n+1}:=-\left(\mathrm{K}_{\mathcal{F} \mathcal{T}} \mathrm{P}_{\mathcal{T}}^{* n}+\mathrm{K}_{\mathcal{F} \mathcal{F}} \mathrm{P}_{\mathcal{F}}^{* n}\right)$, and $\left(\mathrm{P}_{\mathcal{T}}^{* n}, \mathrm{P}_{\mathcal{F}}^{* n}\right)$ are the components of the predicted pressure $\hat{p}_{h}^{* n}$. Since the matrix $\mathrm{M}_{\mathcal{T} \mathcal{T}}+\beta \Delta t^{2} \mathrm{~K}_{\mathcal{T} \mathcal{T}}$ is blockdiagonal, the cell unknown $A_{\mathcal{T}}^{n+1} \in V_{\mathcal{T}}^{k^{\prime}}$ can be eliminated locally in (38), leading to a global 
transmission problem coupling only the face unknown $\mathrm{A}_{\mathcal{F}}^{n+1} \in V_{\mathcal{F} 0}^{k}$. This process is known in the literature as static condensation.

An important property of the HHO-Newmark scheme is an energy balance which is the timediscrete counterpart of (30). For all $n \in\{0, \ldots, N\}$, we define the discrete energy

$$
\hat{E}^{n}:=\frac{1}{2}\left\|v_{\mathcal{T}}^{n}\right\|_{L^{2}\left(\frac{1}{\kappa} ; \Omega\right)}^{2}+\frac{1}{2}\left\|\boldsymbol{G}_{\mathcal{T}}\left(\hat{p}_{h}^{n}\right)\right\|_{\boldsymbol{L}^{2}\left(\frac{1}{\rho} ; \Omega\right)}^{2}+\frac{1}{2}\left|\hat{p}_{h}^{n}\right|_{S}^{2}+\delta \Delta t^{2}\left\|a_{\mathcal{T}}^{n}\right\|_{L^{2}\left(\frac{1}{\kappa} ; \Omega\right)}^{2},
$$

with $\delta:=\frac{1}{4}(2 \beta-\gamma)$, i.e., $\delta=0$ for the standard choice $\beta=\frac{1}{4}, \gamma=\frac{1}{2}$ in the implicit case, and $\delta=-\frac{1}{8}$ in the explicit case (leapfrog scheme with $\beta=0, \gamma=\frac{1}{2}$ ).

Lemma 3.3 (Energy balance, HHO-Newmark scheme). Let $\left(\hat{p}_{h}^{n}, \hat{v}_{h}^{n}, \hat{a}_{h}^{n}\right)_{1 \leq n \leq N}$ solve the HHONewmark scheme (34). Let $\hat{E}^{n}$ be defined in (39). Assume for simplicity that $\gamma=\frac{1}{2}$. The following energy balance holds true for all $n \in\{2, \ldots, N\}$ :

$$
\hat{E}^{n}=\hat{E}^{1}+\sum_{m=1}^{n-1} \frac{1}{2}\left(f\left(t^{m+1}\right)+f\left(t^{m}\right), p_{\mathcal{T}}^{m+1}-p_{\mathcal{T}}^{m}\right)_{L^{2}(\Omega)} .
$$

Proof. Owing to the structure of the HHO discretization, it is possible to adapt the usual arguments for Newmark schemes (see, e.g., [34]). Let $n \in\{1, \ldots, N-1\}$. Since the discrete bilinear form $b_{h}$ is symmetric, we infer that

$$
\frac{1}{2} b_{h}\left(\hat{p}_{h}^{n+1}, \hat{p}_{h}^{n+1}\right)-\frac{1}{2} b_{h}\left(\hat{p}_{h}^{n}, \hat{p}_{h}^{n}\right)=\frac{1}{2} b_{h}\left(\hat{p}_{h}^{n+1}+\hat{p}_{h}^{n}, \hat{p}_{h}^{n+1}-\hat{p}_{h}^{n}\right) .
$$

Considering the first equation in (34) at $t^{n}$ and at $t^{n+1}$, each tested against $\hat{q}_{h}:=\hat{p}_{h}^{n+1}-\hat{p}_{h}^{n}$, and summing the results, we infer that $\frac{1}{2} b_{h}\left(\hat{p}_{h}^{n+1}, \hat{p}_{h}^{n+1}\right)-\frac{1}{2} b_{h}\left(\hat{p}_{h}^{n}, \hat{p}_{h}^{n}\right)=-\Delta_{1}^{n}+\Delta_{2}^{n}$ with

$$
\begin{aligned}
\Delta_{1}^{n} & :=\frac{1}{2}\left(a_{\mathcal{T}}^{n+1}+a_{\mathcal{T}}^{n}, p_{\mathcal{T}}^{n+1}-p_{\mathcal{T}}^{n}\right)_{L^{2}\left(\frac{1}{\kappa} ; \Omega\right)}, \\
\Delta_{2}^{n} & :=\frac{1}{2}\left(f\left(t^{n+1}\right)+f\left(t^{n}\right), p_{\mathcal{T}}^{n+1}-p_{\mathcal{T}}^{n}\right)_{L^{2}(\Omega)} .
\end{aligned}
$$

The term $\Delta_{1}^{n}$ is rewritten by using the second and third equations in (34). Straightforward manipulations (using $\gamma=\frac{1}{2}$ for simplicity) lead to

$$
p_{\mathcal{T}}^{n+1}-p_{\mathcal{T}}^{n}=\frac{1}{2} \Delta t\left(v_{\mathcal{T}}^{n+1}+v_{\mathcal{T}}^{n}\right)+2 \delta \Delta t^{2}\left(a_{\mathcal{T}}^{n+1}-a_{\mathcal{T}}^{n}\right)
$$

whence we infer that

$$
\Delta_{1}^{n}=\frac{1}{2}\left(v_{\mathcal{T}}^{n+1}-v_{\mathcal{T}}^{n}, v_{\mathcal{T}}^{n+1}+v_{\mathcal{T}}^{n}\right)_{L^{2}\left(\frac{1}{\kappa} ; \Omega\right)}+\delta \Delta t^{2}\left(a_{\mathcal{T}}^{n+1}+a_{\mathcal{T}}^{n}, a_{\mathcal{T}}^{n+1}-a_{\mathcal{T}}^{n}\right)_{L^{2}\left(\frac{1}{\kappa} ; \Omega\right)}
$$

Since $b_{h}\left(\hat{q}_{h}, \hat{q}_{h}\right)=\left\|\boldsymbol{G}_{\mathcal{T}}\left(\hat{q}_{h}\right)\right\|_{\boldsymbol{L}^{2}\left(\frac{1}{\rho} ; \Omega\right)}^{2}+\left|\hat{q}_{h}^{n}\right|_{S}^{2}$ for all $\hat{q}_{h} \in \hat{V}_{h 0}$, rearranging the terms implies that $\hat{E}^{n+1}-\hat{E}^{n}=\Delta_{2}^{n}$. This concludes the proof.

Remark 3.4 (Energy for HHO-leapfrog scheme). In the case $\beta=0, \gamma=\frac{1}{2}$, a slightly different form of the energy balance property can be obtained by defining

$$
\check{E}^{n}:=\frac{1}{2}\left\|\tilde{v}_{\mathcal{T}}^{n}\right\|_{L^{2}\left(\frac{1}{\kappa} ; \Omega\right)}^{2}+\frac{1}{2} b_{h}\left(\hat{p}_{h}^{n+1}, \hat{p}_{h}^{n}\right),
$$

with $\tilde{v}_{\mathcal{T}}^{n}:=\frac{1}{\Delta t}\left(p_{\mathcal{T}}^{n+1}-p_{\mathcal{T}}^{n}\right)$. This leads to $\check{E}^{n}-\check{E}^{n-1}=\frac{1}{2}\left(f\left(t^{n}\right), p_{\mathcal{T}}^{n+1}-p_{\mathcal{T}}^{n-1}\right)_{L^{2}(\Omega)}$ for all $n \in\{1, \ldots, N-1\}$. One can verify that $\check{E}^{n}=\hat{E}^{n}+\frac{1}{2}\left(f\left(t^{n}\right), p_{\mathcal{T}}^{n+1}-p_{\mathcal{T}}^{n}\right)_{L^{2}(\Omega)}$, so that $\check{E}^{n}=\hat{E}^{n}$ in the absence of external sources (i.e., $f:=0$ ). 


\section{HHO discretization for the first-order wave equation}

In this section, we present the space semi-discrete and the fully discrete HHO schemes for the first-order wave equation. The temporal discretization is performed by using singly-diagonally implicit and explicit Runge-Kutta schemes (SDIRK and ERK).

\subsection{Space semi-discrete scheme}

Recall that the unknowns in the first-order formulation of the wave equation are the velocity $v$ and the dual variable $\boldsymbol{\sigma}$. In the space semi-discrete HHO scheme, one approximates $v$ by a hybrid unknown $\hat{v}_{h} \in C^{1}\left(\bar{J} ; \hat{V}_{h 0}\right)$ and $\boldsymbol{\sigma}$ by a cellwise unknown $\boldsymbol{\sigma}_{\mathcal{T}} \in C^{1}\left(\bar{J} ; \boldsymbol{W}_{\mathcal{T}}\right)$ (recall that $\left.\boldsymbol{W}_{\mathcal{T}}:=\mathrm{X}_{T \in \mathcal{T}_{h}} \mathbb{P}^{k}\left(T ; \mathbb{R}^{d}\right)\right)$. The space semi-discrete problem reads as follows: For all $t \in \bar{J}$,

$$
\left\{\begin{aligned}
\left(\partial_{t} \boldsymbol{\sigma}_{\mathcal{T}}(t), \boldsymbol{\tau}_{\mathcal{T}}\right)_{\boldsymbol{L}^{2}(\rho ; \Omega)}-\left(\boldsymbol{G}_{\mathcal{T}}\left(\hat{v}_{h}(t)\right), \boldsymbol{\tau}_{\mathcal{T}}\right)_{\boldsymbol{L}^{2}(\Omega)} & =0 \\
\left(\partial_{t} v_{\mathcal{T}}(t), w_{\mathcal{T}}\right)_{L^{2}\left(\frac{1}{\kappa} ; \Omega\right)}+\left(\boldsymbol{\sigma}_{\mathcal{T}}(t), \boldsymbol{G}_{\mathcal{T}}\left(\hat{w}_{h}\right)\right)_{\boldsymbol{L}^{2}(\Omega)}+\tilde{s}_{h}\left(\hat{v}_{h}(t), \hat{w}_{h}\right) & =\left(f(t), w_{\mathcal{T}}\right)_{L^{2}(\Omega)}
\end{aligned}\right.
$$

for all $\left(\boldsymbol{\tau}_{\mathcal{T}}, \hat{w}_{h}\right) \in \boldsymbol{W}_{\mathcal{T}} \times \hat{V}_{h 0}$, with

$$
\tilde{s}_{h}\left(\hat{v}_{h}, \hat{w}_{h}\right):=\sum_{T \in \mathcal{T}_{h}} \tilde{\tau}_{\partial T}\left(S_{\partial T}\left(\hat{v}_{T}\right), S_{\partial T}\left(\hat{w}_{T}\right)\right)_{L^{2}(\partial T)},
$$

recalling that $S_{\partial T}$ is defined by either (19) in the equal-order case $\left(k^{\prime}=k\right)$ or $(20)$ in the mixedorder case $\left(k^{\prime}=k+1\right)$. Moreover $\tilde{\tau}_{\partial T}>0$ is a stabilization parameter which will be taken equal to $\tilde{\tau}_{\partial T}:=\frac{c}{\kappa}=\frac{1}{\rho c}$ (in short, $\tilde{\tau}_{\partial T}=\mathcal{O}(1)$ ) or to $\tilde{\tau}_{\partial T}:=\frac{c}{\kappa} \frac{\ell_{\Omega}}{h_{T}}=\frac{1}{\rho c} \frac{\ell_{\Omega}}{h_{T}}$ (in short, $\tilde{\tau}_{\partial T}=\mathcal{O}\left(h_{T}^{-1}\right)$ ). The initial conditions for (41) only concern $\boldsymbol{\sigma}_{\mathcal{T}}$ and $v_{\mathcal{T}}$ and are as follows:

$$
\boldsymbol{\sigma}_{\mathcal{T}}(\cdot, t=0)=\frac{1}{\rho} \boldsymbol{G}_{\mathcal{T}}\left(\hat{I}_{h}\left(p_{0}\right)\right), \quad v_{\mathcal{T}}(\cdot, t=0)=\Pi_{\mathcal{T}}^{k^{\prime}}\left(v_{0}\right) .
$$

The boundary condition on $v$ is encoded in the fact that $\hat{v}_{h}(t) \in \hat{V}_{h 0}$ for all $t \in \bar{J}$.

Remark 4.1 (Stabilization). The stabilization parameter $\tau_{\partial T}$ in the second-order formulation has unit equal to $\left[\frac{\mathrm{m}^{2}}{\mathrm{~kg}}\right]$, whereas in the first-order formulation the unit of $\tilde{\tau}_{\partial T}$ is $\left[\frac{\mathrm{sm}^{2}}{\mathrm{~kg}}\right]$.

Lemma 4.2 (Space semi-discrete energy balance). Let $\left(\boldsymbol{\sigma}_{\mathcal{T}}, \hat{v}_{h}\right) \in C^{1}\left(\bar{J} ; \boldsymbol{W}_{\mathcal{T}} \times \hat{V}_{h 0}\right)$ solve (41)(43) and define the time-dependent space semi-discrete energy functional as

$$
\hat{E}^{m i x}(t):=\frac{1}{2}\left\|v_{\mathcal{T}}\right\|_{L^{2}\left(\frac{1}{\kappa} ; \Omega\right)}^{2}+\frac{1}{2}\left\|\boldsymbol{\sigma}_{\mathcal{T}}\right\|_{L^{2}(\rho ; \Omega)}^{2} .
$$

Then the following energy balance equation holds true for all $t \in J$ :

$$
\hat{E}^{m i x}(t)+\int_{0}^{t} \tilde{s}_{h}\left(\hat{v}_{h}(s), \hat{v}_{h}(s)\right) d s=\hat{E}^{m i x}(0)+\int_{0}^{t}\left(f(s), v_{\mathcal{T}}(s)\right)_{L^{2}(\Omega)} d s .
$$

Proof. Testing the first equation in (41) against $\boldsymbol{\tau}_{\mathcal{T}}:=\boldsymbol{\sigma}_{\mathcal{T}}(t)$ and the second equation against $\hat{w}_{h}:=\hat{v}_{h}(t)$, and summing the results, we infer that for all $t \in J$,

$$
\frac{d}{d t} \hat{E}^{\text {mix }}(t)+\tilde{s}_{h}\left(\hat{v}_{h}(t), \hat{v}_{h}(t)\right)=\left(f(t), v_{\mathcal{T}}(t)\right)_{L^{2}(\Omega)} .
$$

The assertion follows by integrating the above identity in time over $(0, t)$ for all $t \in J$. 
Remark 4.3 (Role of stabilization). We observe that in the second-order formulation, the stabilization modifies the energy (adds a positive contribution to it), whereas in the first-order formulation the stabilization does not modify the energy but adds a dissipative contribution to the energy balance. As the mesh is refined, both the energy modification and the dissipative contribution tend to zero for smooth solutions.

Recall that $N_{\mathcal{T}}^{k^{\prime}}:=\operatorname{dim}\left(V_{\mathcal{T}}^{k^{\prime}}\right), N_{\mathcal{F}}^{k}:=\operatorname{dim}\left(V_{\mathcal{F} 0}^{k}\right)$ and that $\left\{\varphi_{i}\right\}_{1 \leq i \leq N_{\mathcal{T}}^{k^{\prime}}}$ and $\left\{\psi_{j}\right\}_{1 \leq j \leq N_{\mathcal{F}}^{k}}$ are bases for $V_{\mathcal{T}}^{k^{\prime}}$ and $V_{\mathcal{F} 0}^{k}$, respectively. Let $M_{\mathcal{T}}^{k}:=\operatorname{dim}\left(\boldsymbol{W}_{\mathcal{T}}\right)$ and $\left\{\boldsymbol{\zeta}_{k}\right\}_{1 \leq k \leq M_{\mathcal{T}}^{k}}$ be the chosen basis for $\boldsymbol{W}_{\mathcal{T}}$. It is natural to build this basis as products of a Cartesian basis vector in $\mathbb{R}^{d}$ and a scalar-valued basis function of the discrete space $V_{\mathcal{T}}^{k}$ so that $M_{\mathcal{T}}^{k}=d N_{\mathcal{T}}^{k}$. Let $\mathrm{Z}_{\mathcal{T}} \in \mathbb{R}^{M_{\mathcal{T}}^{k}}$ and $\left(\mathrm{V}_{\mathcal{T}}, \mathrm{V}_{\mathcal{F}}\right) \in \mathbb{R}^{N_{\mathcal{T}}^{k^{\prime}} \times N_{\mathcal{F}}^{k}}$ be the (time-dependent) component vectors of $\boldsymbol{\sigma}_{\mathcal{T}} \in \boldsymbol{W}_{\mathcal{T}}$ and $\hat{v}_{h} \in \hat{V}_{h 0}$, respectively. Let $\mathrm{M}_{\mathcal{T} \mathcal{T}}^{\boldsymbol{\sigma}}$ be the mass matrix associated with the inner product in $\boldsymbol{L}^{2}(\rho ; \Omega)$ and the basis functions $\left\{\boldsymbol{\zeta}_{k}\right\}_{1 \leq k \leq M_{\mathcal{T}}^{k}}$, and recall that $\mathbf{M}_{\mathcal{T} \mathcal{T}}$ is the mass matrix associated with the inner product in $L^{2}\left(\frac{1}{\kappa} ; \Omega\right)$ and the basis functions $\left\{\varphi_{i}\right\}_{1 \leq i \leq N_{\mathcal{T}}^{k^{\prime}}}$. Let $\mathrm{S}_{\mathcal{T} \mathcal{T}}, \mathrm{S}_{\mathcal{T} \mathcal{F}}, \mathrm{S}_{\mathcal{F} \mathcal{T}}, \mathrm{S}_{\mathcal{F} \mathcal{F}}$ be the four blocks composing the matrix representing the stabilization bilinear form $\tilde{s}_{h}$, i.e., $\mathrm{S}_{\mathcal{T} \mathcal{T}, i j}:=$ $\tilde{s}_{h}\left(\left(\varphi_{j}, 0\right),\left(\varphi_{i}, 0\right)\right), \mathrm{S}_{\mathcal{T} \mathcal{F}, i j}:=\tilde{s}_{h}\left(\left(0, \psi_{j}\right),\left(\varphi_{i}, 0\right)\right)$, and so on. Let $\mathrm{G}_{\mathcal{T}} \in \mathbb{R}^{M_{\mathcal{T}}^{k} \times N_{\mathcal{T}}^{k^{\prime}}}$ and $\mathrm{G}_{\mathcal{F}} \in$ $\mathbb{R}^{M_{\mathcal{T}}^{k} \times N_{\mathcal{F}}^{k}}$ be the (rectangular) matrices representing the gradient reconstruction operator $\boldsymbol{G}_{\mathcal{T}}$, i.e., $\boldsymbol{G}_{\mathcal{T}, k i}:=\left(\boldsymbol{\zeta}_{k}, \boldsymbol{G}_{\mathcal{T}}\left(\varphi_{i}, 0\right)\right)_{\boldsymbol{L}^{2}(\Omega)}$ and $\boldsymbol{G}_{\mathcal{F}, k j}:=\left(\boldsymbol{\zeta}_{k}, \boldsymbol{G}_{\mathcal{T}}\left(0, \psi_{j}\right)\right)_{\boldsymbol{L}^{2}(\Omega)}$. The algebraic realization of (41) is as follows: For all $t \in \bar{J}$,

$$
\left[\begin{array}{ccc}
\mathrm{M}_{\mathcal{T} \mathcal{T}}^{\sigma} & 0 & 0 \\
0 & \mathrm{M}_{\mathcal{T} \mathcal{T}} & 0 \\
0 & 0 & 0
\end{array}\right]\left[\begin{array}{c}
\partial_{t} \mathrm{Z}_{\mathcal{T}}(t) \\
\partial_{t} \mathrm{~V}_{\mathcal{T}}(t) \\
\bullet
\end{array}\right]+\left[\begin{array}{ccc}
0 & -\mathrm{G}_{\mathcal{T}} & -\mathrm{G}_{\mathcal{F}} \\
\mathrm{G}_{\mathcal{T}}^{\dagger} & \mathrm{S}_{\mathcal{T} \mathcal{T}} & \mathrm{S}_{\mathcal{T F}} \\
\mathrm{G}_{\mathcal{F}}^{\dagger} & \mathrm{S}_{\mathcal{F} \mathcal{T}} & \mathrm{S}_{\mathcal{F} \mathcal{F}}
\end{array}\right]\left[\begin{array}{c}
\mathrm{Z}_{\mathcal{T}}(t) \\
\mathrm{V}_{\mathcal{T}}(t) \\
\mathrm{V}_{\mathcal{F}}(t)
\end{array}\right]=\left[\begin{array}{c}
0 \\
\mathrm{~F}_{\mathcal{T}}(t) \\
0
\end{array}\right]
$$

where the bullet stands for $\partial_{t} \vee_{\mathcal{F}}(t)$ which is irrelevant owing to the structure of the mass matrix. Notice that the third equation in (46) implies that

$$
\mathrm{S}_{\mathcal{F} \mathcal{F}} \mathrm{V}_{\mathcal{F}}(t)=-\left(\mathrm{G}_{\mathcal{F}}^{\dagger} \mathrm{Z}_{\mathcal{T}}(t)+\mathrm{S}_{\mathcal{F} \mathcal{T}} \mathrm{V}_{\mathcal{T}}(t)\right),
$$

and that the submatrix $S_{\mathcal{F} \mathcal{F}}$ is symmetric positive-definite. This submatrix is additionally blockdiagonal in the mixed-order case $\left(k^{\prime}=k+1\right)$, but this property is lost in the equal-order case $\left(k^{\prime}=k\right)$ because the use of the reconstruction operator in the stabilization couples all the faces belonging to the same cell.

\subsection{Fully discrete scheme}

A rather natural way to discretize in time the space semi-discrete problem (41) is to use a Runge-Kutta (RK) time-stepping scheme. RK schemes are defined by a set of coefficients, $\left\{a_{i j}\right\}_{1 \leq i, j \leq s},\left\{b_{i}\right\}_{1 \leq i \leq s},\left\{c_{i}\right\}_{1 \leq i \leq s}$, where $s \geq 1$ is the number of stages. These coefficients are usually represented in the literature by the Butcher tableau. We consider explicit RK schemes (ERK) where the matrix $\left\{a_{i j}\right\}_{1 \leq i, j \leq s}$ is strictly lower-triangular (see (48), left) and singly diagonally implicit RK schemes (SDIRK) where the matrix $\left\{a_{i j}\right\}_{1 \leq i, j \leq s}$ is lower-triangular with $a_{11}=\ldots=a_{s s}:=a_{*}$ (see (48), right). The corresponding Butcher tableaux are as follows (more specific numerical examples are given in Section 5):

$$
\begin{array}{c|cccc}
c_{1} & 0 & \ldots & \ldots & 0 \\
c_{2} & a_{21} & 0 & \cdots & 0 \\
\vdots & \vdots & \ddots & \ddots & \vdots \\
c_{s} & a_{s 1} & \cdots & a_{s, s-1} & 0 \\
\hline & b_{1} & \cdots & b_{s-1} & b_{s}
\end{array}
$$$$
\begin{array}{c|cccc}
c_{1} & a_{*} & 0 & \cdots & 0 \\
c_{2} & a_{21} & a_{*} & \ddots & 0 \\
\vdots & \vdots & \ddots & \ddots & \vdots \\
c_{s} & a_{s 1} & \cdots & a_{s, s-1} & a_{*} \\
\hline & b_{1} & \cdots & b_{s-1} & b_{s}
\end{array}
$$ 
The implementation of HHO-RK schemes is as follows. For simplicity, we only consider the algebraic realization of the schemes. For all $n \in\{1, \ldots, N\}$, given $\left(\mathrm{Z}_{\mathcal{T}}^{n-1}, \mathrm{~V}_{\mathcal{T}}^{n-1}\right)$ from the previous time step or the initial condition and letting $\mathrm{F}_{\mathcal{T}}^{n-1+c_{j}}:=\mathrm{F}_{\mathcal{T}}\left(t_{n-1}+c_{j} \Delta t\right)$ for all $1 \leq j \leq s$, one computes the intermediate values $\left\{\left(\mathrm{Z}_{\mathcal{T}}^{n, i}, \mathrm{~V}_{\mathcal{T}}^{n, i}\right)\right\}_{1 \leq i \leq s}$ sequentially. For ERK schemes, one proceeds as follows:

1. Set $\left(\mathrm{Z}_{\mathcal{T}}^{n, 1}, \mathrm{~V}_{\mathcal{T}}^{n, 1}\right):=\left(\mathrm{Z}_{\mathcal{T}}^{n-1}, \mathrm{~V}_{\mathcal{T}}^{n-1}\right)$ and solve $\mathrm{S}_{\mathcal{F} \mathcal{F}} \mathrm{V}_{\mathcal{F}}^{n, 1}=-\left(\mathrm{G}_{\mathcal{F}}^{\dagger} \mathrm{Z}_{\mathcal{T}}^{n, 1}+\mathrm{S}_{\mathcal{F} \mathcal{T}} \mathrm{V}_{\mathcal{T}}^{n, 1}\right)$

2. If $s \geq 2$, solve sequentially for all $2 \leq i \leq s$,

$$
\begin{aligned}
{\left[\begin{array}{cc}
\mathrm{M}_{\mathcal{T} \mathcal{T}}^{\boldsymbol{\sigma}} & 0 \\
0 & \mathrm{M}_{\mathcal{T} \mathcal{T}}
\end{array}\right]\left[\begin{array}{l}
\mathrm{Z}_{\mathcal{T}}^{n, i} \\
\mathrm{~V}_{\mathcal{T}}^{n, i}
\end{array}\right]=} & {\left[\begin{array}{cc}
\mathrm{M}_{\mathcal{T} \mathcal{T}}^{\boldsymbol{\sigma}} & 0 \\
0 & \mathrm{M}_{\mathcal{T} \mathcal{T}}
\end{array}\right]\left[\begin{array}{l}
\mathrm{Z}_{\mathcal{T}}^{n-1} \\
\mathrm{~V}_{\mathcal{T}}^{n-1}
\end{array}\right] } \\
& +\Delta t \sum_{j=1}^{i-1} a_{i j}\left(\left[\begin{array}{c}
0 \\
\mathrm{~F}_{\mathcal{T}}^{n-1+c_{j}}
\end{array}\right]-\left[\begin{array}{ccc}
0 & -\mathrm{G}_{\mathcal{T}} & -\mathrm{G}_{\mathcal{F}} \\
\mathrm{G}_{\mathcal{T}}^{\dagger} & \mathrm{S}_{\mathcal{T} \mathcal{T}} & \mathrm{S}_{\mathcal{T} \mathcal{F}}
\end{array}\right]\left[\begin{array}{l}
\mathrm{Z}_{\mathcal{T}}^{n, j} \\
\mathrm{~V}_{\mathcal{T}}^{n, j} \\
\mathrm{~V}_{\mathcal{F}}^{n, j}
\end{array}\right]\right)
\end{aligned}
$$

and $\mathrm{S}_{\mathcal{F} \mathcal{F}} \mathrm{V}_{\mathcal{F}}^{n, i}=-\left(\mathrm{G}_{\mathcal{F}}^{\dagger} \mathrm{Z}_{\mathcal{T}}^{n, i}+\mathrm{S}_{\mathcal{F} \mathcal{T}} \mathrm{V}_{\mathcal{T}}^{n, i}\right)$

3. Finally set

$$
\begin{aligned}
{\left[\begin{array}{cc}
\mathrm{M}_{\mathcal{T} \mathcal{T}}^{\sigma} & 0 \\
0 & \mathrm{M}_{\mathcal{T} \mathcal{T}}
\end{array}\right]\left[\begin{array}{l}
\mathrm{Z}_{\mathcal{T}}^{n} \\
\mathrm{~V}_{\mathcal{T}}^{n}
\end{array}\right]:=} & {\left[\begin{array}{cc}
\mathrm{M}_{\mathcal{T} \mathcal{T}}^{\sigma} & 0 \\
0 & \mathrm{M}_{\mathcal{T} \mathcal{T}}
\end{array}\right]\left[\begin{array}{l}
\mathrm{Z}_{\mathcal{T}}^{n-1} \\
\mathrm{~V}_{\mathcal{T}}^{n-1}
\end{array}\right] } \\
& +\Delta t \sum_{j=1}^{s} b_{j}\left(\left[\begin{array}{c}
0 \\
\mathrm{~F}_{\mathcal{T}}^{n-1+c_{j}}
\end{array}\right]-\left[\begin{array}{ccc}
0 & -\mathrm{G}_{\mathcal{T}} & -\mathrm{G}_{\mathcal{F}} \\
\mathrm{G}_{\mathcal{T}}^{\dagger} & \mathrm{S}_{\mathcal{T} \mathcal{T}} & \mathrm{S}_{\mathcal{T} \mathcal{F}}
\end{array}\right]\left[\begin{array}{l}
\mathrm{Z}_{\mathcal{T}}^{n, j} \\
\mathrm{~V}_{\mathcal{T}}^{n, j} \\
\mathrm{~V}_{\mathcal{F}}^{n, j}
\end{array}\right]\right)
\end{aligned}
$$

We emphasize that the HHO-ERK scheme is particularly effective in the mixed-order case $\left(k^{\prime}=\right.$ $k+1)$ since the submatrix $\mathbf{S}_{\mathcal{F} F}$ is then block-diagonal, whereas the scheme is somewhat less attractive in the equal-order case $\left(k^{\prime}=k\right)$ because a sparse linear solver is needed to invert $\mathbf{S}_{\mathcal{F} \mathcal{F}}$.

For HHO-SDIRK schemes, the face unknowns are not handled separately and there is a sparse linear system to be solved at each substage. One proceeds as follows:

1. Solve sequentially for all $1 \leq i \leq s$,

$$
\begin{aligned}
{\left[\begin{array}{ccc}
\mathrm{M}_{\mathcal{T} \mathcal{T}}^{\boldsymbol{\sigma}} & 0 & 0 \\
0 & \mathrm{M}_{\mathcal{T} \mathcal{T}} & 0 \\
0 & 0 & 0
\end{array}\right]\left[\begin{array}{c}
\mathrm{Z}_{\mathcal{T}}^{n, i} \\
\mathrm{~V}_{\mathcal{T}}^{n, i} \\
\bullet
\end{array}\right]=\left[\begin{array}{ccc}
\mathrm{M}_{\mathcal{T} \mathcal{T}}^{\boldsymbol{\sigma}} & 0 & 0 \\
0 & \mathrm{M}_{\mathcal{T} \mathcal{T}} & 0 \\
0 & 0 & 0
\end{array}\right]\left[\begin{array}{c}
\mathrm{Z}_{\mathcal{T}}^{n-1} \\
\mathrm{~V}_{\mathcal{T}}^{n-1} \\
\bullet
\end{array}\right] } \\
+\Delta t \sum_{j=1}^{i} a_{i j}\left(\left[\begin{array}{c}
0 \\
\mathrm{~F}_{\mathcal{T}}^{n-1+c_{j}} \\
0
\end{array}\right]-\left[\begin{array}{ccc}
0 & -\mathrm{G}_{\mathcal{T}} & -\mathrm{G}_{\mathcal{F}} \\
\mathrm{G}_{\mathcal{T}}^{\dagger} & \mathrm{S}_{\mathcal{T} \mathcal{T}} & \mathrm{S}_{\mathcal{T} \mathcal{F}} \\
\mathrm{G}_{\mathcal{F}}^{\dagger} & \mathrm{S}_{\mathcal{F} \mathcal{T}} & \mathrm{S}_{\mathcal{F} \mathcal{F}}
\end{array}\right]\left[\begin{array}{c}
\mathrm{Z}_{\mathcal{T}}^{n, j} \\
\mathrm{~V}_{\mathcal{T}}^{n, j} \\
\mathrm{~V}_{\mathcal{F}}^{n, j}
\end{array}\right]\right)
\end{aligned}
$$

Notice that this is a linear system for the triple $\left(\mathrm{Z}_{\mathcal{T}}^{n, i}, \mathrm{~V}_{\mathcal{T}}^{n, i}, \mathrm{~V}_{\mathcal{F}}^{n, i}\right)$ (which appears on both the left- and right-hand sides). An important observation in (51) is that the upper $2 \times 2$ submatrix associated with the cell unknowns $\left(\mathrm{Z}_{\mathcal{T}}^{n, i}, \mathrm{~V}_{\mathcal{T}}^{n, i}\right)$ is block-diagonal (this is indeed the case for $M_{\mathcal{T} \mathcal{T}}^{\sigma}, M_{\mathcal{T} \mathcal{T}}, G_{\mathcal{T}}$, and $S_{\mathcal{T} \mathcal{T}}$ ). Hence, static condensation can be performed in (51) leading to a global transmission problem coupling only the components of the face unknown $\mathrm{V}_{\mathcal{F}}^{n, i}$. 
2. Finally set

$$
\begin{aligned}
{\left[\begin{array}{cc}
\mathrm{M}_{\mathcal{T} \mathcal{T}}^{\sigma} & 0 \\
0 & \mathrm{M}_{\mathcal{T} \mathcal{T}}
\end{array}\right]\left[\begin{array}{l}
\mathrm{Z}_{\mathcal{T}}^{n} \\
\mathrm{~V}_{\mathcal{T}}^{n}
\end{array}\right]:=} & {\left[\begin{array}{cc}
\mathrm{M}_{\mathcal{T} \mathcal{T}}^{\sigma} & 0 \\
0 & \mathrm{M}_{\mathcal{T} \mathcal{T}}
\end{array}\right]\left[\begin{array}{l}
\mathrm{Z}_{\mathcal{T}}^{n-1} \\
\mathrm{~V}_{\mathcal{T}}^{n-1}
\end{array}\right] } \\
& +\Delta t \sum_{j=1}^{s} b_{j}\left(\left[\begin{array}{c}
0 \\
\mathrm{~F}_{\mathcal{T}}^{n-1+c_{j}}
\end{array}\right]-\left[\begin{array}{ccc}
0 & -\mathrm{G}_{\mathcal{T}} & -\mathrm{G}_{\mathcal{F}} \\
\mathrm{G}_{\mathcal{T}}^{\dagger} & \mathrm{S}_{\mathcal{T} \mathcal{T}} & \mathrm{S}_{\mathcal{T} \mathcal{F}}
\end{array}\right]\left[\begin{array}{l}
\mathrm{Z}_{\mathcal{T}}^{n, j} \\
\mathrm{~V}_{\mathcal{T}}^{n, j} \\
\mathrm{~V}_{\mathcal{F}}^{n, j}
\end{array}\right]\right)
\end{aligned}
$$

Remark 4.4 (Energy balance). We do not explore theoretically the energy balance associated with HHO-ERK and HHO-SDIRK schemes. We refer the reader to [12] for some energy identities satisfied by some two-stage and three-stage ERK schemes.

\subsection{Bridge to HDG formulations}

Inspired by [19], we relate the above space semi-discrete HHO formulation for the first-order wave equation to the space semi-discrete HDG formulation devised in [40]. Let us consider the local spaces $\boldsymbol{W}_{T}^{k}:=\mathbb{P}^{k}\left(T ; \mathbb{R}^{d}\right), V_{T}^{k^{\prime}}:=\mathbb{P}^{k^{\prime}}(T ; \mathbb{R})$ for all $T \in \mathcal{T}_{h}$, and $M_{F}^{k}:=\mathbb{P}^{k}(F ; \mathbb{R})$ for all $F \in \mathcal{F}_{h}$. The unknowns in the space semi-discrete HHO problem (41) are the (time-dependent) quantities $\boldsymbol{\sigma}_{\mathcal{T}}=\left(\boldsymbol{\sigma}_{T}\right)_{T \in \mathcal{T}_{h}}$ with $\boldsymbol{\sigma}_{T} \in \boldsymbol{W}_{T}^{k}, v_{\mathcal{T}}=\left(v_{T}\right)_{T \in \mathcal{T}_{h}}$ with $v_{T} \in V_{T}^{k^{\prime}}$ and $v_{\mathcal{F}}=\left(\lambda_{F}\right)_{F \in \mathcal{F}_{h}}$ with $\lambda_{F} \in V_{F}^{k}$. We also set $\lambda_{\partial T}:=\left(\lambda_{F}\right)_{F \in \mathcal{F}_{\partial T}}$. The Dirichlet condition is enforced by setting $\lambda_{F}=0$ for all $F \in \mathcal{F}_{h}^{\partial}$. The $\lambda$-notation is introduced to facilitate the analogy with the HDG formulation.

Taking a test function in the first equation of (41) with a support localized in a single mesh cell $T \in \mathcal{T}_{h}$, and using the definition (17) of the gradient reconstruction $\boldsymbol{G}_{T}$, we infer that

$$
\left(\partial_{t} \boldsymbol{\sigma}_{T}(t), \boldsymbol{\tau}_{T}\right)_{L^{2}(\rho ; T)}+\left(v_{T}(t), \nabla \cdot \boldsymbol{\tau}_{T}\right)_{L^{2}(T)}-\sum_{F \in \mathcal{F}_{\partial T}}\left(\lambda_{F}(t), \boldsymbol{\tau}_{T} \cdot \boldsymbol{n}_{T}\right)_{L^{2}(F)}=0
$$

for all $\boldsymbol{\tau}_{T} \in \boldsymbol{W}_{T}^{k}$. This is the first equation in [40, Equ. (5)].

Before considering the second equation, we rewrite in a slightly different form the stabilization bilinear form $\tilde{s}_{h}$. In the mixed-order case $\left(k^{\prime}=k+1\right)$, we notice that we have

$$
\tilde{s}_{h}\left(\hat{v}_{h}, \hat{w}_{h}\right)=\sum_{T \in \mathcal{T}_{h}} \sum_{F \in \mathcal{F}_{\partial T}} \tilde{\tau}_{\partial T}\left(\Pi_{F}^{k}\left(v_{T \mid F}-\lambda_{F}\right), w_{T \mid F}-\mu_{F}\right)_{L^{2}(F)},
$$

where $w_{\mathcal{T}}=\left(w_{T}\right)_{T \in \mathcal{T}_{h}}$ and $w_{\mathcal{F}}=\left(\mu_{F}\right)_{F \in \mathcal{F}_{h}}$ (again, the $\mu$-notation is introduced to facilitate the analogy with the HDG formulation). In the equal-order case $\left(k^{\prime}=k\right)$, we first observe from (19) that $S_{\partial T}\left(\hat{v}_{T}\right)=S_{\partial T}^{\prime}\left(v_{T \mid \partial T}-\lambda_{\partial T}\right)$ with $S_{\partial T}^{\prime}: V_{\partial T}^{k} \rightarrow V_{\partial T}^{k}$ such that $S_{\partial T}^{\prime}(\delta):=$ $\Pi_{\partial T}^{k}\left(\delta+\left(\left(I-\Pi_{T}^{k}\right) R_{T}(0, \delta)\right)_{\mid \partial T}\right)$. Letting $\left(S_{\partial T}^{\prime}\right)^{*}: V_{\partial T}^{k} \rightarrow V_{\partial T}^{k}$ denote the adjoint operator of $S_{\partial T}^{\prime}$ with respect to the inner product of $L^{2}(\partial T)$, we obtain

$$
\tilde{s}_{h}\left(\hat{v}_{h}, \hat{w}_{h}\right)=\sum_{T \in \mathcal{T}_{h}} \sum_{F \in \mathcal{F}_{\partial T}} \tilde{\tau}_{\partial T}\left(\left(\left(S_{\partial T}^{\prime}\right)^{*} \circ S_{\partial T}^{\prime}\right)\left(v_{T \mid \partial T}-\lambda_{\partial T}\right), w_{T \mid F}-\mu_{F}\right)_{L^{2}(F)} .
$$

The expressions (54) and (55) can be unified by writing

$$
\tilde{s}_{h}\left(\hat{v}_{h}, \hat{w}_{h}\right)=\sum_{T \in \mathcal{T}_{h}} \sum_{F \in \mathcal{F}_{\partial T}} \tilde{\tau}_{\partial T}\left(\Phi_{T, F}\left(v_{T \mid \partial T}-\lambda_{\partial T}\right), w_{T \mid F}-\mu_{F}\right)_{L^{2}(F)},
$$


with

$$
\Phi_{T, F}\left(v_{T \mid \partial T}-\lambda_{\partial T}\right):= \begin{cases}\Pi_{F}^{k}\left(v_{T \mid F}-\lambda_{F}\right) & \text { (mixed-order case) } \\ \left(\left(\left(S_{\partial T}^{\prime}\right)^{*} \circ S_{\partial T}^{\prime}\right)\left(v_{T \mid \partial T}-\lambda_{\partial T}\right)\right)_{\mid F} & \text { (equal-order case) } .\end{cases}
$$

Notice that $\Phi_{T, F}\left(v_{T \mid \partial T}-\lambda_{\partial T}\right)$ only depends on $\lambda_{F}$ in the mixed-order case, whereas it depends collectively on $\lambda_{\partial T}$ in the equal-order case.

We can now return to the second equation in $(41)$. Considering first a test function $\left(w_{\mathcal{T}}, 0\right)$ with $w_{\mathcal{T}}$ supported on a single mesh cell $T \in \mathcal{T}_{h}$, and using again the definition (17) of the gradient reconstruction $\boldsymbol{G}_{T}$ together with the above rewriting of the stabilization bilinear form $\tilde{s}_{h}$, we infer that

$$
\left(\partial_{t} v_{T}(t), w_{T}\right)_{L^{2}\left(\frac{1}{\kappa} ; T\right)}+\left(\boldsymbol{\sigma}_{T}(t), \nabla w_{T}\right)_{L^{2}(T)}-\sum_{F \in \mathcal{F}_{\partial T}}\left(\hat{\sigma}_{T, F}(t), w_{T}\right)_{L^{2}(F)}=\left(f(t), w_{T}\right)_{L^{2}(T)},
$$

for all $w_{T} \in V_{T}^{k^{\prime}}$, with the numerical flux trace (we omit the time dependency)

$$
\hat{\sigma}_{T, F}:=\boldsymbol{\sigma} \cdot \boldsymbol{n}_{T}-\tilde{\tau}_{\partial T} \Phi_{T, F}\left(v_{T \mid \partial T}-\lambda_{\partial T}\right) .
$$

In other words, we recover the second equation in [40, Equ. (5)] and the definition of the numerical flux trace is similar to [40, Equ. (8)]. More specifically, in the mixed-order case the numerical flux trace only depends on $\lambda_{F}$ as is usually the case in HDG methods, i.e., we have $\hat{\sigma}_{T, F}:=$ $\boldsymbol{\sigma} \cdot \boldsymbol{n}_{T}-\tilde{\tau}_{\partial T} \Pi_{F}^{k}\left(v_{T \mid F}-\lambda_{F}\right)$, whereas in the equal-order case $\hat{\sigma}_{T, F}$ depends more collectively on $\lambda_{\partial T}$. Finally, we consider a test function in $(41)$ of the form $\left(0, w_{\mathcal{F}}\right)$ with $w_{\mathcal{F}}$ supported on a single mesh interface $F \in \mathcal{F}_{h}^{\circ}$ with $F:=\partial T_{-} \cap \partial T_{+}$. We infer that $\left(\hat{\sigma}_{T_{-}, F}+\hat{\sigma}_{T_{+}, F}, \mu_{F}\right)_{L^{2}(F)}=0$ for all $\mu_{F} \in V_{F}^{k}$, and since $\hat{\sigma}_{T_{ \pm}, F} \in V_{F}^{k}$, this ensures the algebraic conservation of the numerical flux trace across the interface $F$.

The devising of HDG-SDIRK schemes has been studied in [40], whereas the devising of HDG-ERK schemes is pursued in [43]. Therein, although written in the HDG language, the key observation is the same as above for HHO-ERK schemes, namely that the stabilization submatrix $\mathrm{S}_{\mathcal{F} \mathcal{F}}$ is block-diagonal for HDG schemes, and this allows for an efficient implementation of HDGERK schemes in terms of only cell unknowns (this is called a DG rewriting of the HDG scheme in [43]). Finally, we observe that the same reasoning as above allows us to connect the fully discrete HHO formulation for the second-order wave equation to the fully discrete HDG formulation devised in [20], up to a different treatment of the source term.

\section{Numerical results}

In this section, we evaluate numerically the various $\mathrm{HHO}$ schemes considered above on two types of test cases: test cases with analytical solution to report convergence rates for smooth solutions, and test cases with contrasted properties to study how the schemes can deal with this situation. We consider both equal-order and mixed-order settings for the HHO discretization. The implementation of HHO methods is discussed in [18] and an open-source software is available (see https://github.com/wareHHOuse/diskpp).

For the temporal discretization of the second-order formulation, we consider the implicit Newmark scheme (see Section 3.2) with parameters $\beta=\frac{1}{4}$ and $\gamma=\frac{1}{2}$ unless stated otherwise. We do not investigate further the leapfrog scheme since, as already observed, the handling of the face unknowns requires the inversion of a sparse matrix at each time step, which hinders the efficiency expected for an explicit time-stepping scheme. For the temporal discretization of 
the first-order formulation, we consider RK schemes as described in Section 4.2. We consider $\operatorname{ERK}(s)$ schemes with $s$-stages of order $s$ with $s \in\{1,2,3,4\}$. Their Butcher tableaux are

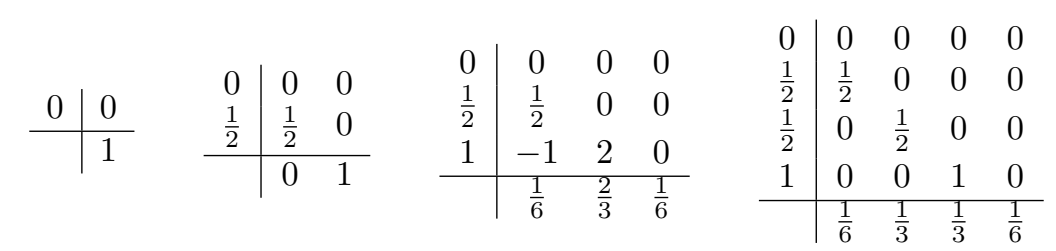

We also consider $\operatorname{SDIRK}(s, s+1)$ schemes with $s$-stages of order $(s+1)$ with $s \in\{1,2,3\}$. Their Butcher tableaux are

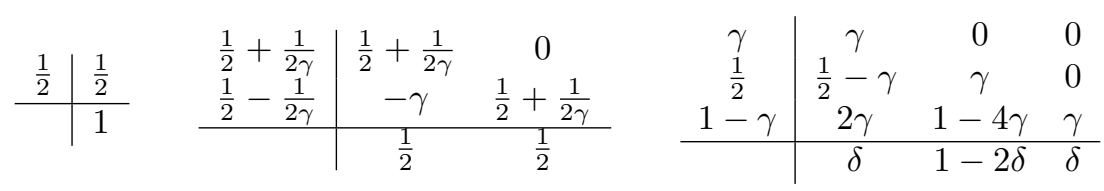

with $\gamma:=\frac{1}{\sqrt{3}}$ for $s=2$, and $\gamma:=\frac{1}{\sqrt{3}} \cos \left(\frac{\pi}{18}\right)+\frac{1}{2}, \delta:=\frac{1}{6(2 \gamma-1)^{2}}$ for $s=3$.

\subsection{Test cases with analytical solutions}

We consider a two-dimensional setting with $\Omega:=(0,1) \times(0,1), T_{\mathrm{f}}:=1, \rho:=1$, and $\kappa:=1$. The data $f, p_{0}$, and $v_{0}$ are chosen according to the following analytic solutions:

1. Quadratic in time, so that the spatial error is the only error component:

$$
p\left(x_{1}, x_{2}, t\right):=t^{2} \sin \left(\pi x_{1}\right) \sin \left(\pi x_{2}\right) .
$$

2. Quadratic in space, so that the temporal error is the only error component:

$$
p\left(x_{1}, x_{2}, t\right):=\sin (\sqrt{2} \pi t) x_{1}\left(1-x_{1}\right) x_{2}\left(1-x_{2}\right) .
$$

3. Non-polynomial in space and in time:

$$
p\left(x_{1}, x_{2}, t\right):=\frac{1}{\sqrt{2} \pi} \sin (\sqrt{2} \pi t) \sin \left(\pi x_{1}\right) \sin \left(\pi x_{2}\right) .
$$

Notice that $f:=0$ in this case.

To verify the convergence rates, we first consider uniformly refined sequences of quadrangular meshes with size $h:=2^{-l}$ and $l \in\{1, \ldots, 6\}$. The time step size is set to $\Delta t:=0.1 \times 2^{-l}$ with $l \in\{0, \ldots, 7\}$. We report the $L^{2}$ and $H^{1}$-errors at the final time. The $L^{2}$-error is evaluated using the cell component of the discrete solution and the $L^{2}$-orthogonal projection of the exact solution onto $V_{\mathcal{T}}^{k^{\prime}}$, whereas the $H^{1}$-error is evaluated using the reconstructed gradient of the discrete solution and the gradient of the exact solution. Notice that the evaluation of the $L^{2}$ error is based on the cell unknowns (as is usual with HHO methods and mixed finite element methods), and differs from the evaluation considered in the context of HDG methods where a post-processed solution has to be computed.

The results for the HHO-Newmark scheme are shown in Figures 1 and 2. We consider the equal-order case $\left(k^{\prime}=k\right)$. Similar results (not shown for brevity) are obtained in the mixed-order case $\left(k^{\prime}=k+1\right)$. We observe that the $L^{2}$-error converges optimally with rate $\mathcal{O}\left(h^{k+2}\right)$ and the 

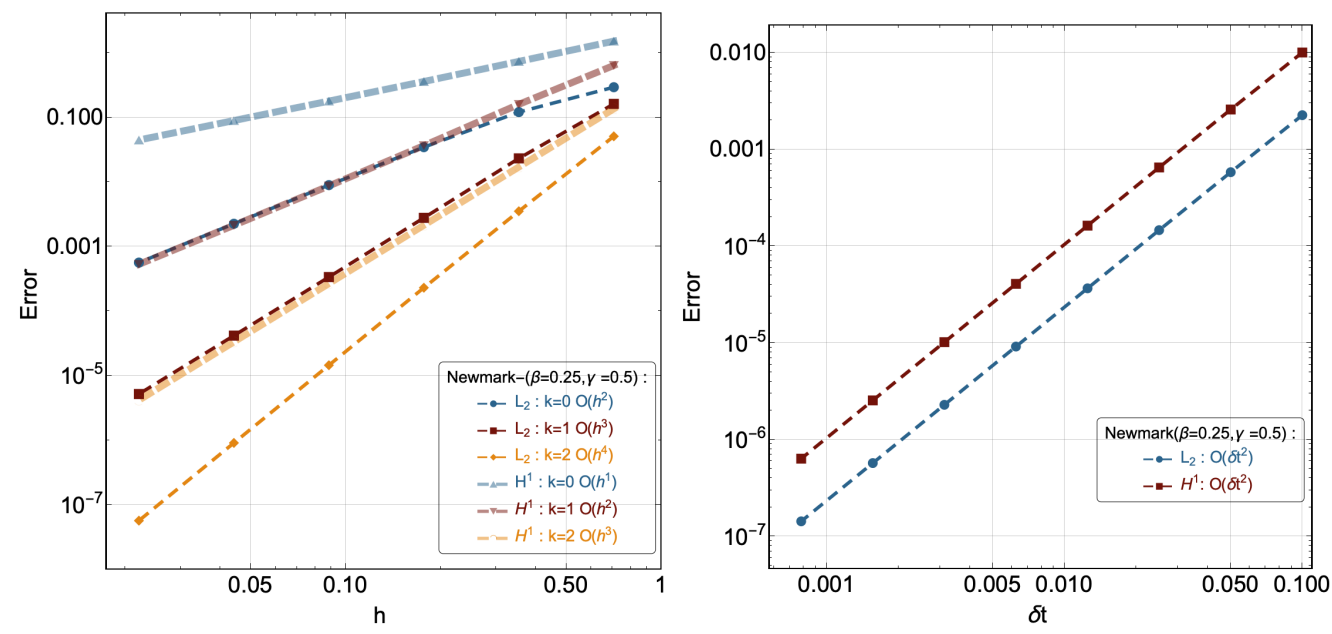

Figure 1: HHO-Newmark scheme, equal-order case: errors as a function of the mesh-size for the analytic solution (60) (left panel) and as a function of the time step for the analytic solution (61) (right panel, $k=2$ ).

$H^{1}$-error converges optimally with rate $\mathcal{O}\left(h^{k+1}\right)$, and, in both cases, second-order convergence in time is observed. Notice that in the left panel of Figure 2, the $L^{2}$-error for $k=2$ on the finest mesh is saturated by the time discretization error. Furthermore, the energy $\hat{E}^{n}$ is exactly conserved in agreement with Lemma 3.3 (see the right panel of Figure 2), and, as expected, this is no longer the case if one keeps the definition (39) of $\hat{E}^{n}$ with $\delta:=0$ but the value of the parameters $(\beta, \gamma)$ is slightly perturbed around the nominal value $\left(\frac{1}{4}, \frac{1}{2}\right)$.

The results for the HHO-SDIRK schemes are reported in Figures 3, 4, and 5. In Figure 3, we show the $L^{2}$ and $H^{1}$-errors as a function of the mesh-size for the analytic solution (60) in the mixed-order case (similar results not shown for brevity are obtained in the equal-order case). The time-stepping scheme is $\operatorname{SDIRK}(2,3)$. The stabilization parameter is $\tilde{\tau}_{\partial T}=\frac{1}{\rho c}=\mathcal{O}(1)$ in the left panel and $\tilde{\tau}_{\partial T}=\frac{1}{\rho c} \frac{\ell_{\Omega}}{h_{T}}=\mathcal{O}\left(h_{T}^{-1}\right)$ in the right panel (see (42)). Interestingly, we see that for both choices, the $H^{1}$-error converges optimally with rate $\mathcal{O}\left(h^{k+1}\right)$, whereas the convergence of the $L^{2}$-error is optimal with rate $\mathcal{O}\left(h^{k+2}\right)$ if $\tilde{\tau}_{\partial T}=\mathcal{O}\left(h_{T}^{-1}\right)$ and suboptimal with rate $\mathcal{O}\left(h^{k+1}\right)$ if $\tilde{\tau}_{\partial T}=\mathcal{O}(1)$. This loss of superconvergence (actually supercloseness) for the $L^{2}$-error when the stabilization parameter is $\mathcal{O}(1)$ is also observed when solving steady diffusion problems. In the left panel of Figure 4, we show the $L^{2}$ and $H^{1}$-errors as a function of the mesh-size for the analytic solution $(62)$ using $\operatorname{SDIRK}(3,4)$ as the time-stepping scheme. We consider the equalorder case and $\tilde{\tau}_{\partial T}=\mathcal{O}\left(h_{T}^{-1}\right)$. Optimal convergence rates are obtained, of order $\mathcal{O}\left(h^{k+1}\right)$ in the $H^{1}$-norm and $\mathcal{O}\left(h^{k+2}\right)$ in the $L^{2}$-norm. In the right panel of Figure 4, we display the $L^{2}$ and $H^{1}$-errors as a function of the time step for the analytic solution (61) using $\operatorname{SDIRK}(s, s+1)$ with $s \in\{1,2,3\}$. For both error norms, we recover the optimal convergence rates of order $\mathcal{O}\left(\Delta t^{s+1}\right)$. In Figure 5, we focus on the relative energy loss using $\operatorname{HHO}-\operatorname{SDIRK}(3,4)$ in the equal-order (left panel) and mixed-order (right panel) cases. The analytical solution is (62). We compare the use of the penalty parameter $\tilde{\tau}_{\partial T}=\mathcal{O}(1)$ (thin lines) and $\tilde{\tau}_{\partial T}=\mathcal{O}\left(h_{T}^{-1}\right)$ (thick lines) for the polynomial degrees $k \in\{0,1,2\}$. Recall that there is no result on exact energy balance for HHO-SDIRK schemes and that the stabilization bilinear form contributes as a dissipative term in the energy balance. Interestingly, we observe that, in both equal-order and mixed-order cases, the relative energy loss is significantly decreased if the polynomial degree $k$ is increased, 

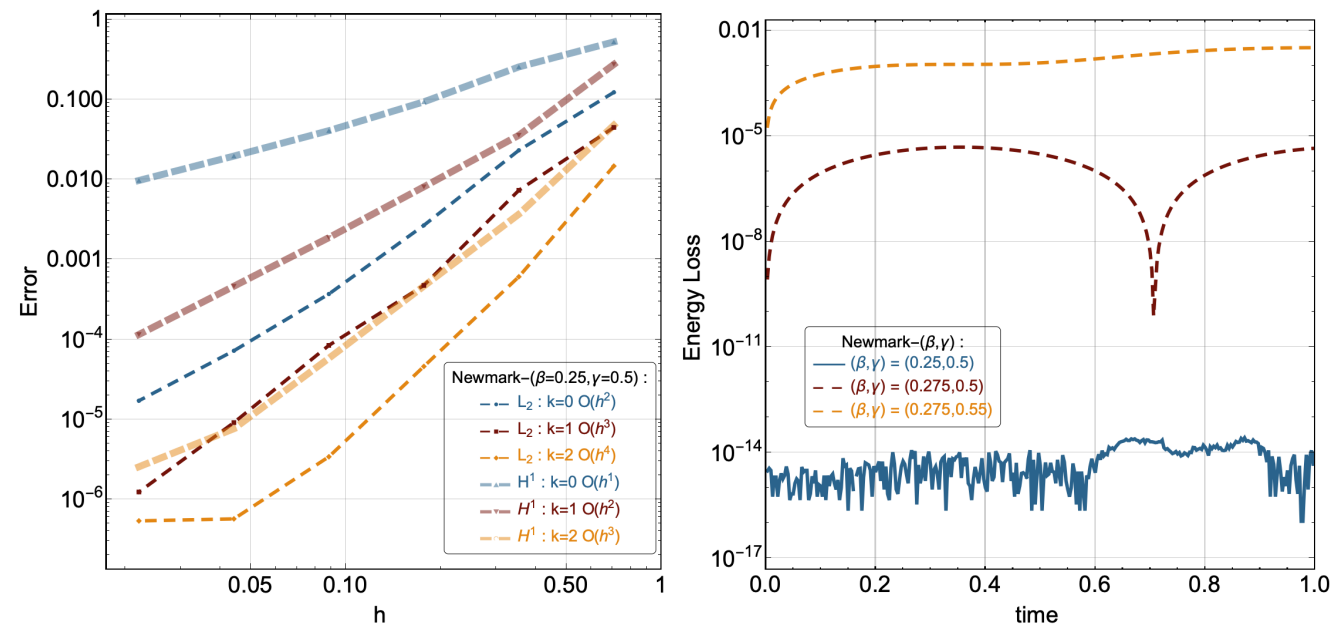

Figure 2: HHO-Newmark scheme, equal-order case: errors as a function of the mesh-size for the analytic solution (62) (left panel, $\Delta t=0.1 \times 2^{-7}$ ) and relative energy loss as a function of time (right panel, $h=2^{-6}, \Delta t=0.1 \times 2^{-7}, k=2$ ).

and that this loss is also decreased if the penalty parameter is tightened from $\tilde{\tau}_{\partial T}=\mathcal{O}(1)$ to $\tilde{\tau}_{\partial T}=\mathcal{O}\left(h_{T}^{-1}\right)$. This latter result may seem a bit surprising at first sight, but it indicates that tightening the penalty parameter decreases the jumps between the traces of the cell unknowns and the face unknowns and that this is the dominant effect so that altogether the dissipative contribution of the stabilization term diminishes as the penalty parameter is increased.

The results for the HHO-ERK(4) scheme are reported in Figures 6 where we consider the mixed-order case, $k \in\{0,1,2\}$, and $\tilde{\tau}_{\partial T}=\mathcal{O}\left(h_{T}^{-1}\right)$ (left panel) or $\tilde{\tau}_{\partial T}=\mathcal{O}(1)$ (right panel). We report the $L^{2}$ and $H^{1}$-errors as a function of the mesh-size for the analytic solution (62). As for HHO-SDIRK, for $\tilde{\tau}_{\partial T}=\mathcal{O}\left(h_{T}^{-1}\right)$ both errors converge optimally with rates $\mathcal{O}\left(h^{k+2}\right)$ and $\mathcal{O}\left(h^{k+1}\right)$, respectively, but for $\tilde{\tau}_{\partial T}=\mathcal{O}(1)$ both errors converge with rates $\mathcal{O}\left(h^{k+1}\right)$. As for HHO-SDIRK, there is no result on exact energy balance for HHO-ERK schemes, and the stabilization bilinear form contributes as a dissipative term in the energy balance. We observe again that increasing the polynomial degree diminishes significantly the relative energy losses (figure omitted for brevity).

\begin{tabular}{|c|cccc|cccc|}
\hline$s \backslash k$ & 0 & 1 & 2 & 3 & 0 & 1 & 2 & 3 \\
\hline \hline 1 & 0.01359 & 0.00793 & 0.00491 & 0.00358 & 0.11851 & 0.05223 & 0.03387 & 0.02293 \\
\hline 2 & 0.04500 & 0.01098 & 0.00549 & 0.00319 & 0.45000 & 0.22500 & 0.12222 & 0.07857 \\
\hline 3 & 0.05000 & 0.01364 & 0.00682 & 0.00398 & 0.50000 & 0.27500 & 0.15714 & 0.09999 \\
\hline 4 & 0.05625 & 0.01500 & 0.00763 & 0.00446 & 0.55000 & 0.33000 & 0.16667 & 0.11250 \\
\hline
\end{tabular}

Table 1: HHO-ERK $(s)$ schemes: stability limit $\beta(s) \mu(k)$ as a function of the number of stages $s \in\{1,2,3,4\}$ and the polynomial degree $k \in\{0,1,2,3\}$ for $\tilde{\tau}_{\partial T}=\frac{1}{\rho c}$. The first four columns concern the equal-order case $\left(k^{\prime}=k\right)$ and the last four columns the mixed-order case $\left(k^{\prime}=k+1\right)$.

Another important aspect of $\operatorname{ERK}(s)$ schemes is their conditional stability. Whenever $\tilde{\tau}_{\partial T}=$ $\mathcal{O}(1)$, we find that the stability limit on the time step on quasi-uniform meshes is of the form

$$
c_{\sharp} \frac{\Delta t}{h} \leq \beta(s) \mu(k),
$$



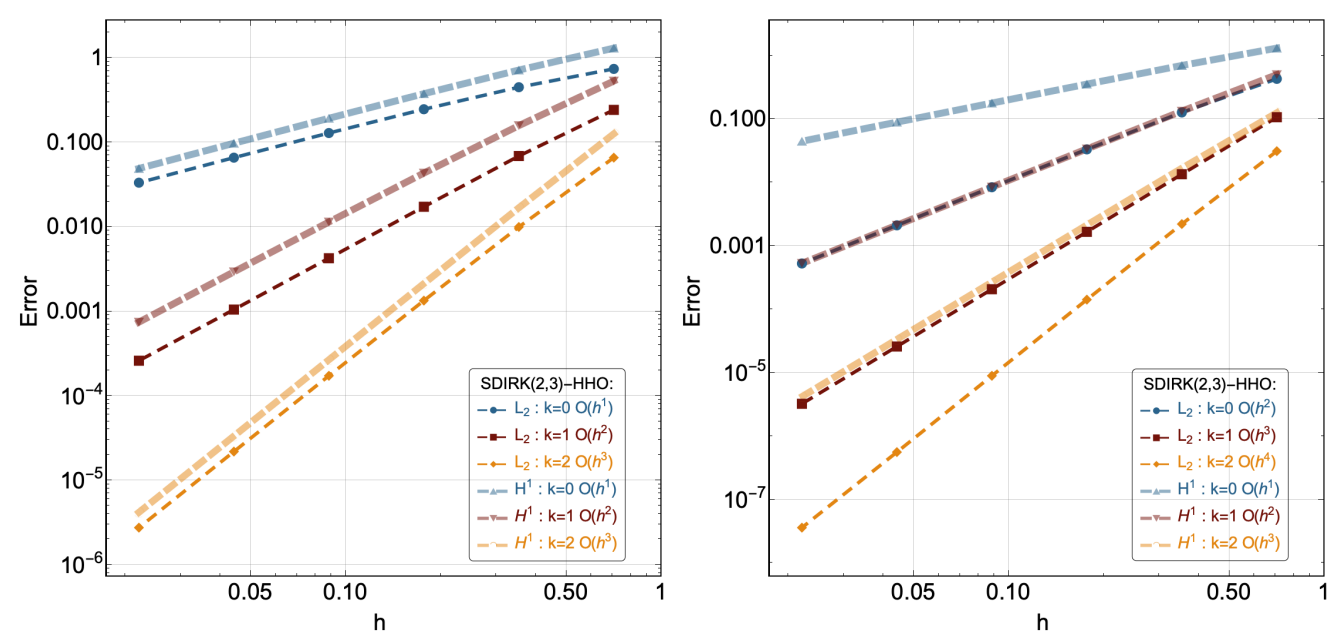

Figure 3: HHO-SDIRK $(2,3)$ scheme, mixed-order case: errors as a function of the mesh-size for the analytic solution (60). The stabilization parameter is $\tilde{\tau}_{\partial T}=\mathcal{O}(1)$ in the left panel and $\tilde{\tau}_{\partial T}=\mathcal{O}\left(h_{T}^{-1}\right)$ in the right panel.

where $c_{\sharp}$ is the largest velocity in the domain, $\beta(s) \approx \mathcal{O}(1)$ is a coefficient that depends on the number of stages (conventionally, $\beta(1)=1$ ), and $\mu(k)$ is a coefficient that depends on the mesh geometry and the polynomial degree $k$ (and is expected to scale as $\left.(k+1)^{-1}\right)$. To compute the coefficient $\mu(k)$, we consider uniform material properties and compute numerically on a sequence of uniform Cartesian meshes of size $h=0.1 \times 2^{-l}, l \in\{0,1,2,3,4\}$, the largest eigenvalue, say $\lambda_{\max }(h, k)$, of the generalized spectral problem $\mathrm{AX}=\lambda \mathrm{MX}$, where $\mathrm{A}$ and $\mathrm{M}$ are the stiffness and mass matrices appearing in the algebraic realization (46) of the space semi-discrete HHO formulation with polynomial degree $k \geq 0$. We find that $\mu(k) \approx\left(h \lambda_{\max }(h, k)\right)^{-1}$ is $h$-independent and its value is reported in the first row of Table 1. The values reported in the other rows (for $s \in\{2,3,4\}$ and $k \in\{0,1,2,3\})$ are estimated by flagging the value of the time step as unstable if a relative energy increase of $\epsilon:=1 \%$ is observed in a time step. These values show that $\beta(s)$ slightly increases with $s$. Finally, we observe that if the stabilization parameter is chosen as $\tilde{\tau}_{\partial T}=\mathcal{O}\left(h_{T}^{-1}\right)$, the stability limit on the time step scales as $h^{2}$ rather than $h$, which makes the method less attractive. This adverse effect can be partially countered by taking $\tilde{\tau}_{\partial T}=\eta \frac{1}{\rho c} \frac{\ell_{\Omega}}{h_{T}}$, where $\eta$ is a small coefficient, e.g., $\eta=0.1$. This strategy is, however, not further explored herein, and in the rest of this work, we always take $\tilde{\tau}_{\partial T}=\frac{1}{\rho c}$ when using $\operatorname{ERK}(s)$ schemes despite the fact that the decay rate of the $L^{2}$-error may not be optimal.

We now consider polygonal meshes to illustrate the capability of the studied schemes to deal with such meshes. Figure 7 shows the first four (out of eight) polygonal meshes used for the convergence tests. These meshes have been generated using the software PolyMesher [44]. The mesh size is determined by the largest distance between two vertices of any polygonal cell. The time step size is $\Delta t=0.1 \times 2^{-5}$ for the implicit schemes (HHO-Newmark and HHO-SDIRK $(3,4)$ ) and $\Delta t=\frac{h}{320}$ for the explicit HHO-ERK(4) scheme. The exact solution is (60). Figure 8 shows that the same optimal convergence rates in the $L^{2}$ and $H^{1}$-norms are obtained as for quadrangular meshes. Similar results (not shown for brevity) are obtained with $\operatorname{HHO}-\operatorname{SDIRK}(3,4)$ in the mixed-order case and with HHO-ERK(4) in the equal-order case. In addition, similar results concerning the energy balance (not shown for brevity) are obtained in all cases. 

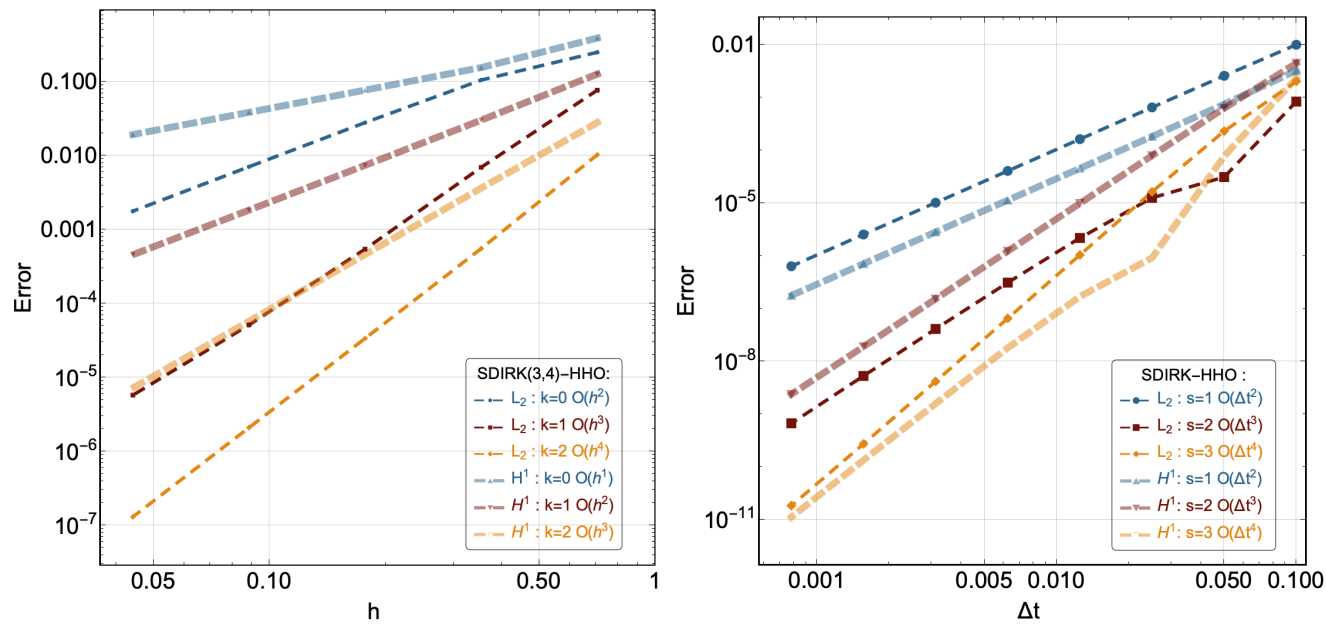

Figure 4: HHO-SDIRK scheme, equal-order case and $\tilde{\tau}_{\partial T}=\mathcal{O}\left(h_{T}^{-1}\right)$ : errors as a function of the mesh-size for the analytic solution (62) using $\operatorname{SDIRK}(3,4)$ (left panel, $\Delta t=0.1 \times 2^{-8}$ ) and errors as a function of the time step for the analytic solution (61) using $\operatorname{SDIRK}(s, s+1)$ with $s \in\{1,2,3\}$ (right panel, $h=2^{-3}, k=3$ ).
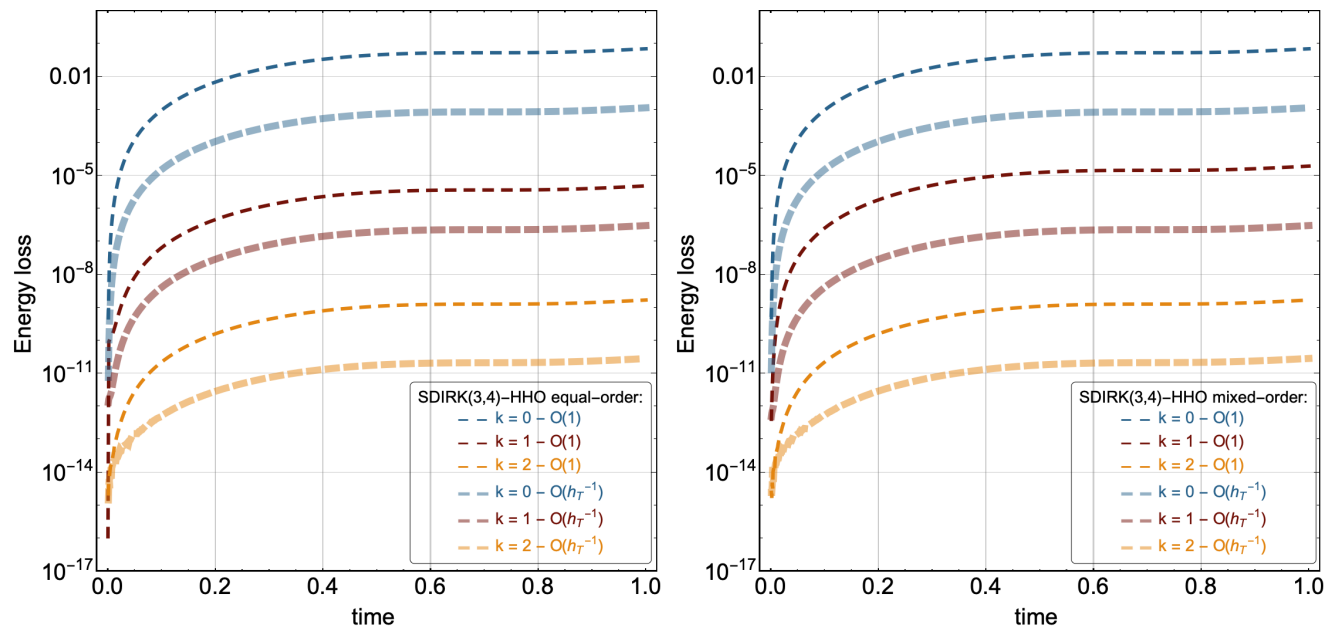

Figure 5: HHO-SDIRK $(3,4)$ scheme: relative energy loss as a function of time in the equal-order (left panel) and mixed-order (right panel) cases. $\Delta t=0.1 \times 2^{-7}$ and $h=2^{-5}$. The analytical solution is (62). 

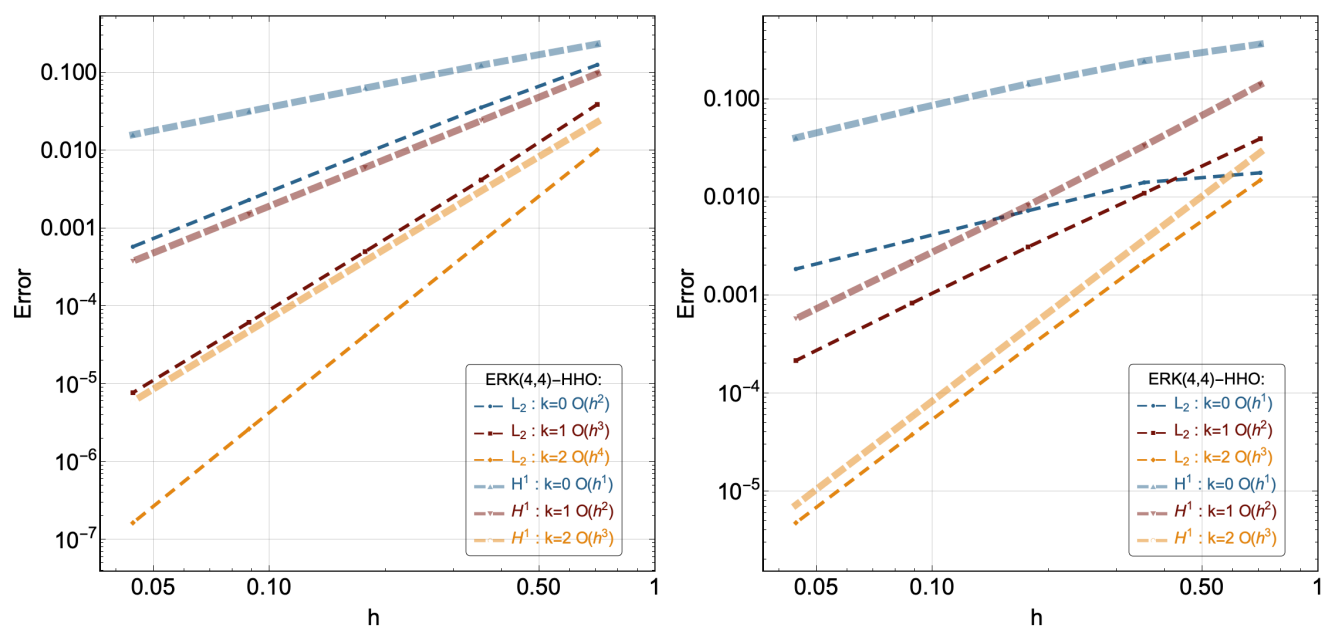

Figure 6: HHO-ERK(4) scheme, mixed-order case, $k \in\{0,1,2\}, \tilde{\tau}_{\partial T}=\mathcal{O}\left(h_{T}^{-1}\right)$ (left panel) and $\tilde{\tau}_{\partial T}=\mathcal{O}(1)$ (right panel): errors as a function of the mesh-size. The time step size is $\Delta t=\frac{h}{320}$, and the analytical solution is (62).
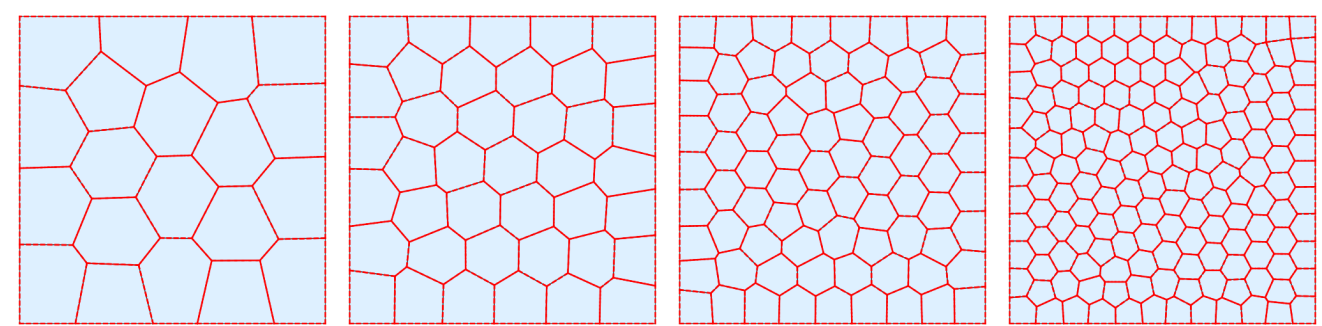

Figure 7: Polygonal meshes: the number of cells is $20 \times 2^{l}$ with $l \in\{0,1,2,3\}$. 

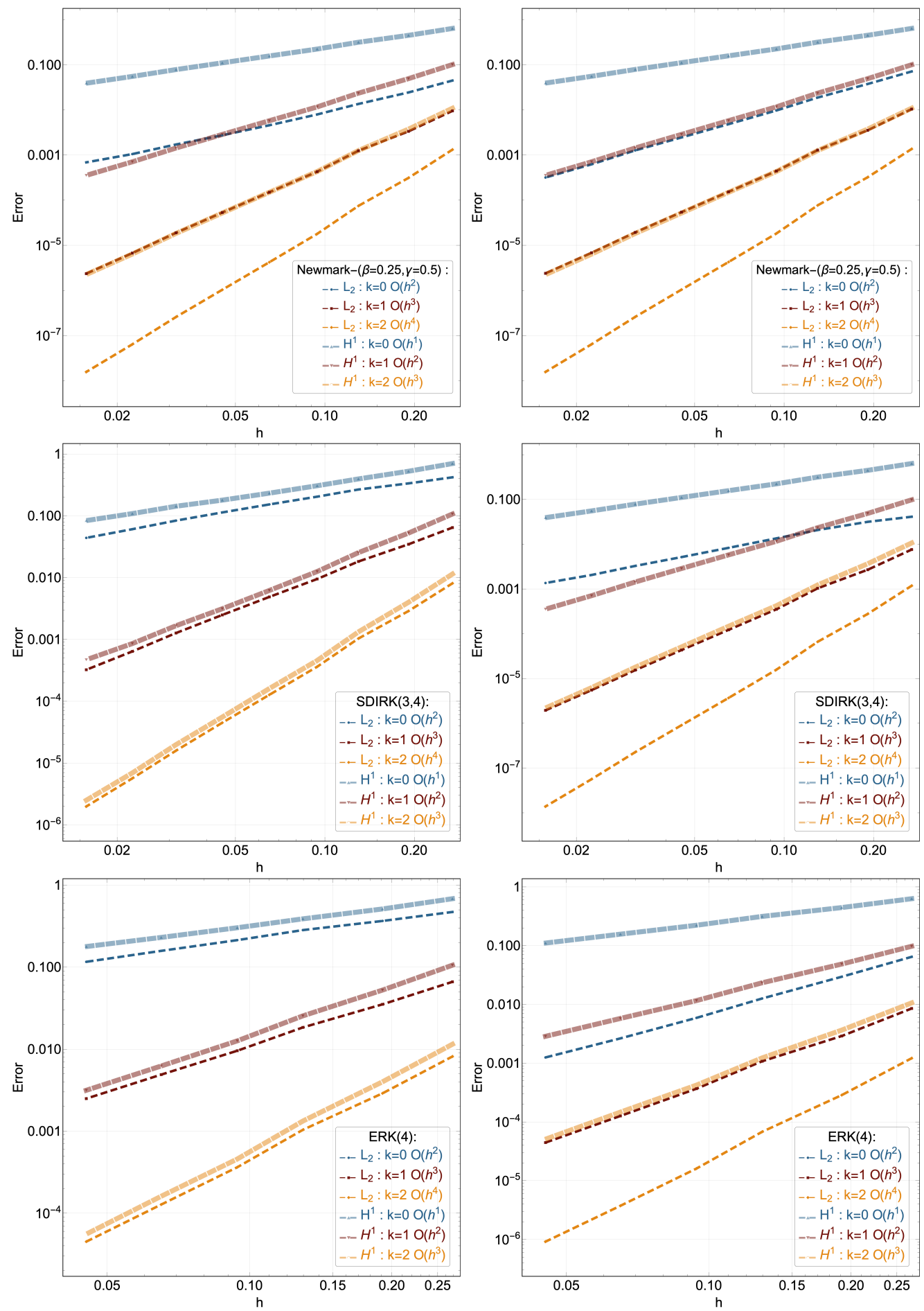

Figure 8: Polygonal meshes: errors as a function of the mesh-size for the analytic solution (60). Top row: HHO-Newmark for equal-order (left panel) and mixed-order (right panel) cases, $\Delta t=$ $0.1 \times 2^{-5}$. Central row: HHO-SDIRK $(3,4)$ scheme, equal-order case, $\tilde{\tau}_{\partial T}=\mathcal{O}(1)$ (left panel) and $\tilde{\tau}_{\partial T}=\mathcal{O}\left(h_{T}^{-1}\right)$ (right panel), $\Delta t=0.1 \times 2^{-5}$. Bottom row: HHO-ERK(4) scheme, mixed-order case, $\tilde{\tau}_{\partial T}=\mathcal{O}(1)$ (left panel) and $\tilde{\tau}_{\partial T}=\mathcal{O}\left(h_{T}^{-1}\right)_{23}$ (right panel), $\Delta t=\frac{h}{320}$. 


\subsection{Test case with geometric heterogeneity}

This test case deals with the scattering of a plane wave by a NACA 0012 airfoil. In this test case, the geometric heterogeneity calls for the use of locally refined meshes around the airfoil, which is particularly challenging for explicit time-stepping schemes since the lowest cell diameter drives the stability limit on the time step. The scattered pressure field is approximated in the truncated domain $\Omega:=(-3.5,2.5) \times(-2.5,2.5)$. The domain $\Omega$ and the locally refined triangulation are presented in Figure 9. The incident plane wave that hits the wingtip is of the form $p_{\text {inc }}(\boldsymbol{x}, t)=\sin (\boldsymbol{k} \cdot \boldsymbol{x}-c\|\boldsymbol{k}\| t)$. We set $c:=1$, the wave number is $\boldsymbol{k}:=(20,0)$, and the final time is $T_{\mathrm{f}}:=2$. The initial conditions for the scattered wave are $p_{0}:=0$ and $v_{0}:=0$. The boundary conditions are of homogeneous Dirichlet type on the external boundary (the final time is sufficiently short to avoid reflections on the outer boundary for this test case), and we enforce the Neumann condition $\nabla p \cdot \boldsymbol{n}=-\nabla p_{\text {inc }} \cdot \boldsymbol{n}$ around the airfoil.

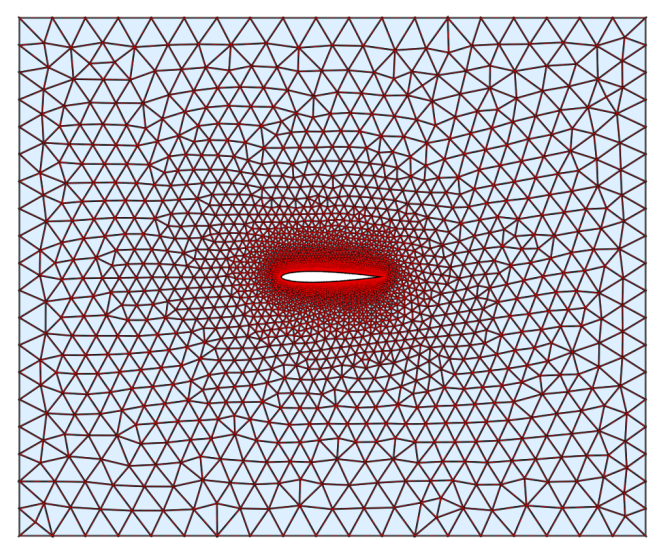

Figure 9: Computational domain and locally refined mesh for the scattering of a plane wave by a NACA 0012 airfoil. The number of cells is 6,297.

The HHO-SDIRK $(3,4)$ scheme is used with $\Delta t:=0.2 \times 2^{-6}$, a mixed-order setting, $\tilde{\tau}_{\partial T}=$ $\mathcal{O}\left(h_{T}^{-1}\right)$ and the mesh in Figure 9 (6,297 cells). The computed velocity distribution (recall that the velocity is defined as the time derivative of the pressure) is presented in Figure 10 for $k:=6$ for the two times $t \in\{1,2\}$. The results are in good qualitative agreement with those presented in [40]. We report in Figure 11 the $\sigma_{x}$-profile as a function of time at a sensor located at the point with coordinates $\left(0, \frac{1}{2}\right)$ (just above the wing tip). The reference solution is obtained with a fine mesh composed of 14,875 cells, still with $k:=6$ and the same time step as above. The error in each curve reported in Figure 11 is normalized by using the reference value 0.2968 . The relative error for $k=6$ is $4.35 \%$, whereas the relative errors for the lower polynomial orders are above $5 \%$. Similar results are obtained with HHO-ERK(4) (not shown for brevity). Finally, we compare HHO-SDIRK $(3,4)$ and HHO-ERK(4) in terms of computational costs, making sure that both methods lead to a relative error below $5 \%$ for the $\sigma_{x}$-component at the above sensor. The cost per time step for HHO-SDIRK $(3,4)$ is 0.8504 seconds and 640 time steps are employed, whereas the cost per time step for HHO-ERK(4) is 0.6903 seconds and 10,240 time steps are employed. Indeed, the refined cells around the airfoil increase the stiffness, and due to the CFL condition, the time step decreases down to $\Delta t:=0.2 \times 2^{-10}(16$ times smaller than for HHO-SDIRK $(3,4))$. In conclusion, for this test case which calls for locally refined meshes, the implicit time-stepping scheme is more cost-effective than the explicit one. 

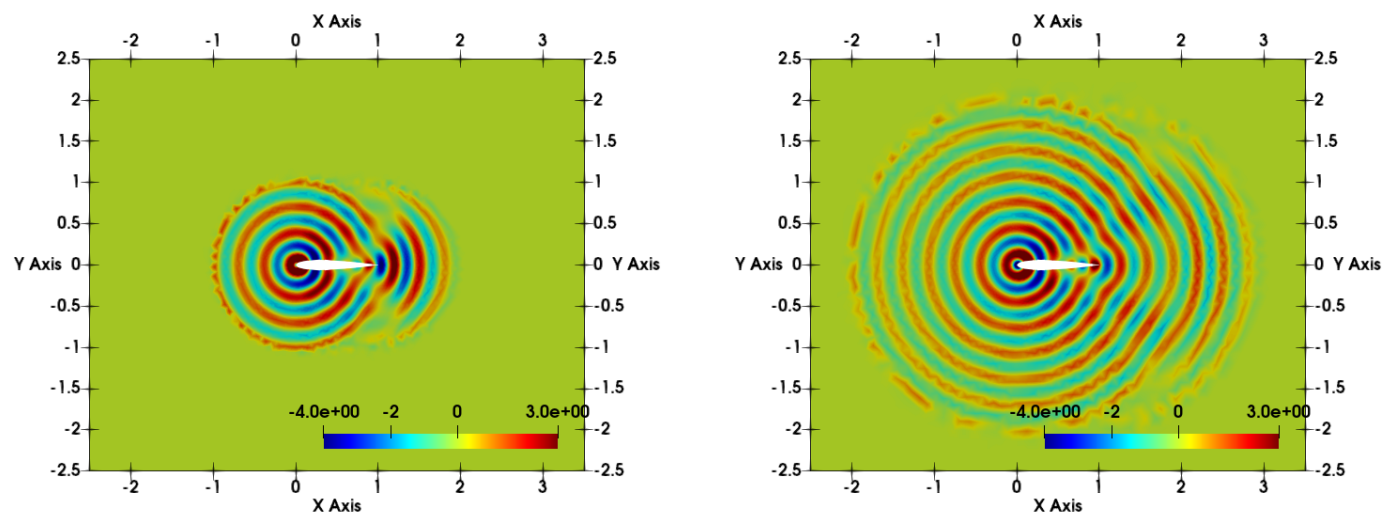

Figure 10: Two-dimensional velocity distributions predicted by the HHO-SDIRK $(3,4)$ scheme with $\tilde{\tau}_{\partial T}=\mathcal{O}\left(h_{T}^{-1}\right)$, mixed-order setting, and $k:=6$. Time values $t=1$ (left panel) and $t=2$ (right panel).

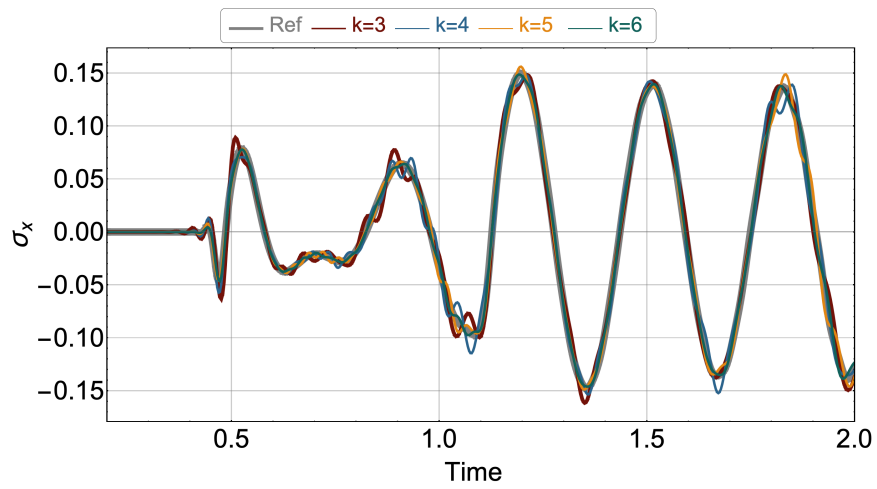

Figure 11: $\sigma_{x}$-component as a function of time predicted at a sensor located at the point with coordinates $\left(0, \frac{1}{2}\right)$ for $k \in\{3,4,5,6\}$. HHO-SDIRK $(3,4)$ scheme with $\tilde{\tau}_{\partial T}=\mathcal{O}\left(h_{T}^{-1}\right)$ and mixedorder setting.

\subsection{Test cases with contrasted properties}

In this section, we consider two test cases of acoustic waves propagating through materials with contrasted properties. The first test case is one-dimensional and an analytic expression of the exact solution is available in the form of a series. The second test case is two-dimensional and there is no analytic solution available (at least in a bounded domain).

The one-dimensional test case has been considered in [37] to evaluate an explicit, secondorder, pseudo-energy conserving scheme. The spatial domain is partitioned as $\bar{\Omega}=\bar{\Omega}_{1} \cup \bar{\Omega}_{2}$ with $\Omega_{1}:=(0,0.5)$ and $\Omega_{2}:=(0.5,1)$. The final time is $T_{\mathrm{f}}:=\frac{1}{2}$, and the wave speeds are $c_{1} \in\{1,2,10,50\}$ and $c_{2}:=1$. The source term is $f:=0$ and the initial conditions are obtained from the following exact solution:

$$
p(x, t):= \begin{cases}\sum_{k \geq 0}\left(\frac{c_{2}-c_{1}}{c_{2}+c_{1}}\right)^{k}\left(p^{*}\left(k+x-c_{1} t\right)-p^{*}\left(k-x-c_{1} t\right)\right), & x \in \Omega_{1}, \\ \left(\frac{2 c_{1}}{c_{2}+c_{1}}\right) \sum_{k \geq 0}\left(\frac{c_{2}-c_{1}}{c_{2}+c_{1}}\right)^{k} p^{*}\left(\frac{c_{1}}{c_{2}}\left(x-\frac{1}{2}\right)+k+\frac{1}{2}-c_{1} t\right), & x \in \Omega_{2},\end{cases}
$$


with $p^{*}(x):=\frac{1}{100} \exp \left(-20\left(x-\frac{1}{5}\right)^{2}\right)$. The initial condition corresponds to a wave located in $\Omega_{1}$ that propagates toward the interface at $x=\frac{1}{2}$, part of the wave is then reflected back to $\Omega_{1}$ whereas part of the wave penetrates into $\Omega_{2}$ where the oscillatory behavior is more pronounced as the velocity contrast is higher. The polynomial order in the HHO method is $k \in\{0,1,2\}$. The mesh size is fixed to $h:=0.1 \times 2^{-5}$ on $\Omega_{1}$ and $h:=0.1 \times 2^{-l}$ on $\Omega_{2}$ with $l \in\{5,6,7,8\}$ for $c_{1} \in\{1,2,10,50\}$, so as to reflect the need to use a finer mesh in $\Omega_{2}$ as the velocity contrast is increased. These meshes are fitted to the material interface located at $x=\frac{1}{2}$. The time step is set to $\Delta t:=0.1 \times 2^{-9}$.
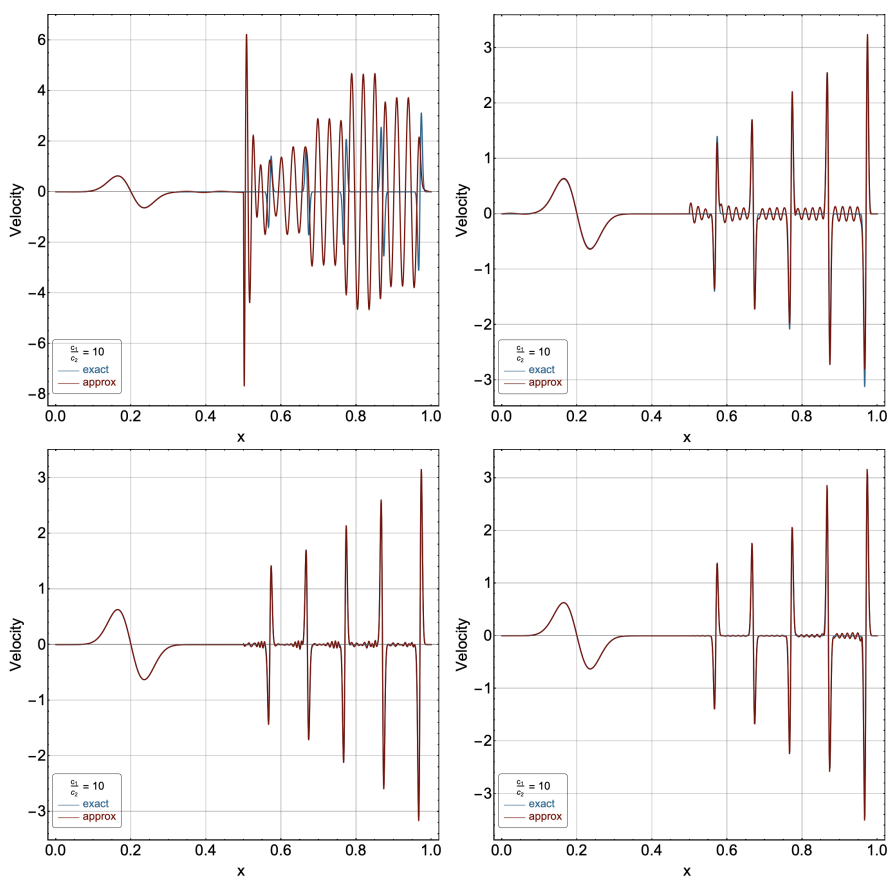

Figure 12: HHO-Newmark scheme, equal-order setting with $k=0$ (top-left), $k=1$ (top-right), $k=2$ (bottom-left), and $k=3$ (bottom-right). The velocity contrast is $\frac{c_{1}}{c_{2}}:=10$ and the time is $t=\frac{1}{2}$.

The results for the HHO-Newmark scheme are presented in Figure 12 for the equal-order setting and the polynomial orders $k \in\{0,1,2,3\}$ at the final time $t=T_{\mathrm{f}}:=\frac{1}{2}$. The velocity contrast is set to $\frac{c_{1}}{c_{2}}:=10$. We observe that the mesh resolution is not sufficient to capture the wave transmission for $k=0$, but the situation is substantially improved for $k=1$ although some dispersion effects remain visible. These effects gradually disappear as the polynomial degree is increased to $k=2$ and $k=3$. The results for the $\operatorname{HHO} \operatorname{SDIRK}(3,4)$ scheme are presented in Figure 13 for the same setting. We can see that overall the accuracy is (much) better than for the HHO-Newmark scheme. In particular, for the polynomial degrees $k \in\{1,2,3\}$, the discrete solution predicted by HHO-SDIRK $(3,4)$ is essentially superimposed to the exact solution. The situation becomes more challenging as the velocity contrast is increased to $\frac{c_{1}}{c_{2}}:=50$. The results presented in Figure 14 are obtained at the shorter final time $T_{\mathrm{f}}:=\frac{1}{4}$ and with the finer mesh-size $h=0.1 \times 2^{-9}$ in $\Omega_{2}$ (the time step is kept to $\Delta t=0.1 \times 2^{-9}$ ). We can see that the wave penetrating into $\Omega_{2}$ is not captured when $k=0$, whereas it is relatively well-captured for $k=1$ and the accuracy improves further for $k=2$ and $k=3$. In Figure 15, we present the relative 

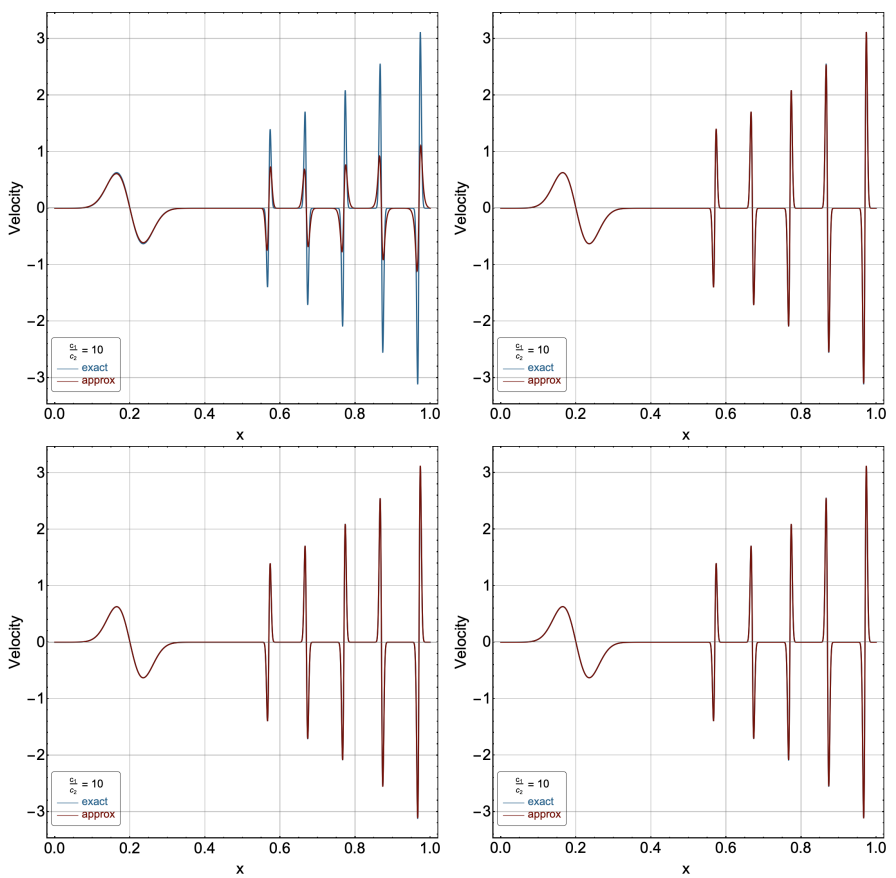

Figure 13: HHO-SDIRK $(3,4)$ scheme, equal-order setting with $k=0$ (top-left), $k=1$ (topright), $k=2$ (bottom-left), and $k=3$ (bottom-right). The velocity contrast is $\frac{c_{1}}{c_{2}}:=10$ and the time is $t=\frac{1}{2}$.

energy loss as a function of time for the $\operatorname{HHO} \operatorname{SDIRK}(3,4)$ scheme, the equal-order setting, the polynomial degrees $k \in\{0,1,2,3\}$, and the velocity contrasts $\frac{c_{1}}{c_{2}} \in\{1,2,10,50\}$. We can see that, for all the values of the velocity contrast, the increase in the polynomial degree is beneficial to the accuracy of the energy balance, and that this effect is more pronounced when the velocity contrast is low. For instance, comparing $k=0$ to $k=3$, the relative energy loss at the final time $T=\frac{1}{2}$ is six orders of magnitude smaller for $\frac{c_{1}}{c_{2}}:=1$ and only a factor of three smaller for $\frac{c_{1}}{c_{2}}:=50$. Finally, we mention that the HHO-ERK $(4)$ scheme was also tested and similar results to those with HHO-SDIRK $(3,4)$ were obtained (not shown for brevity). 

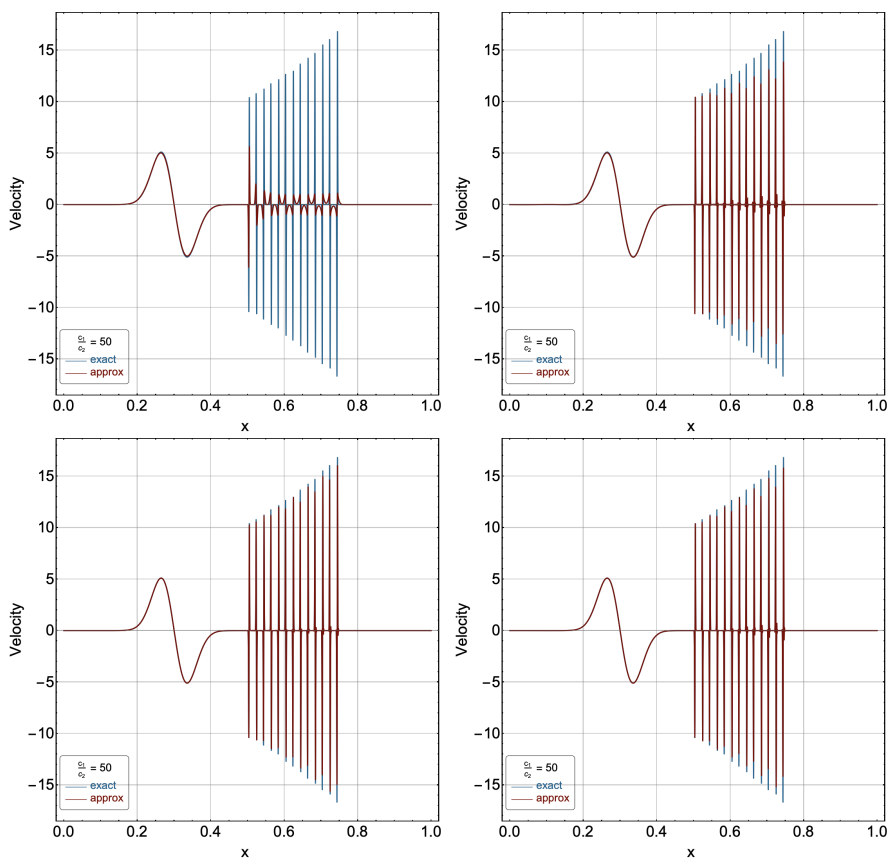

Figure 14: HHO-SDIRK $(3,4)$ scheme, equal-order setting with $k=0$ (top-left), $k=1$ (topright), $k=2$ (bottom-left), and $k=3$ (bottom-right). The velocity contrast is $\frac{c_{1}}{c_{2}}:=50$, the final time is shortened to $T_{\mathrm{f}}:=\frac{1}{4}$, and the mesh-size is refined to $h=0.1 \times 2^{-9}$, whereas the time step is kept to $\Delta t=0.1 \times 2^{-9}$. 

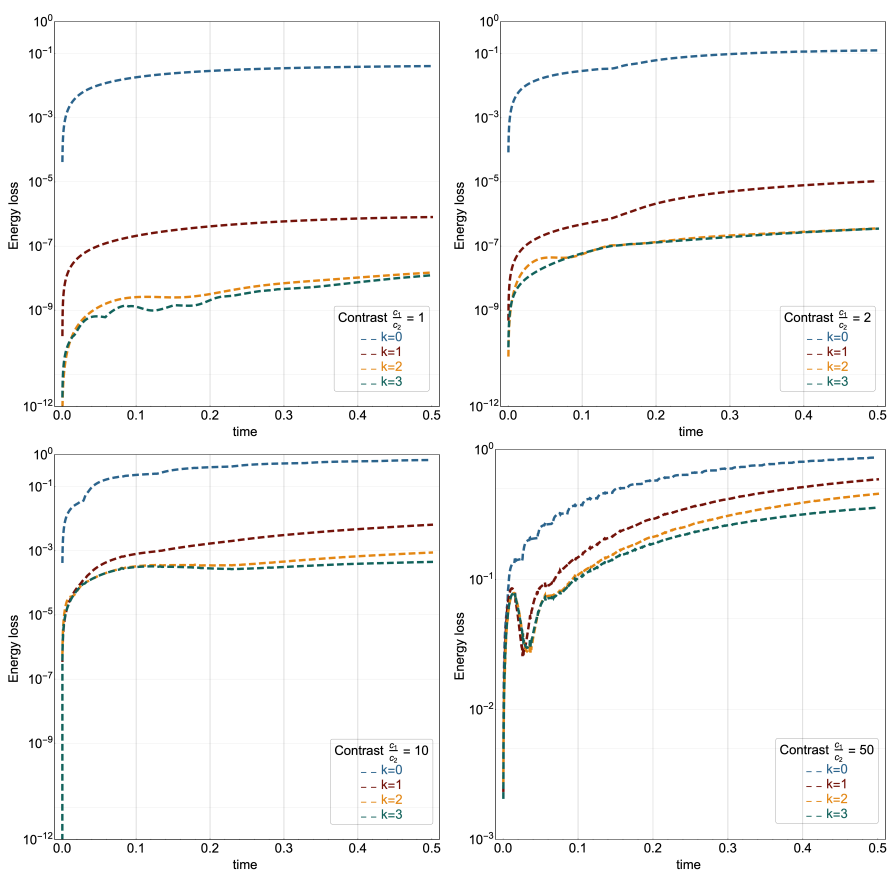

Figure 15: HHO-SDIRK $(3,4)$ scheme: relative energy loss as a function of time, equal-order setting with $k \in\{0,1,2,3\}$. The contrast factor is $\frac{c_{1}}{c_{2}}:=1$ (top-left), $\frac{c_{1}}{c_{2}}:=2$ (top-right), $\frac{c_{1}}{c_{2}}:=10$ (bottom-left), and $\frac{c_{1}}{c_{2}}:=50$ (bottom-right). 

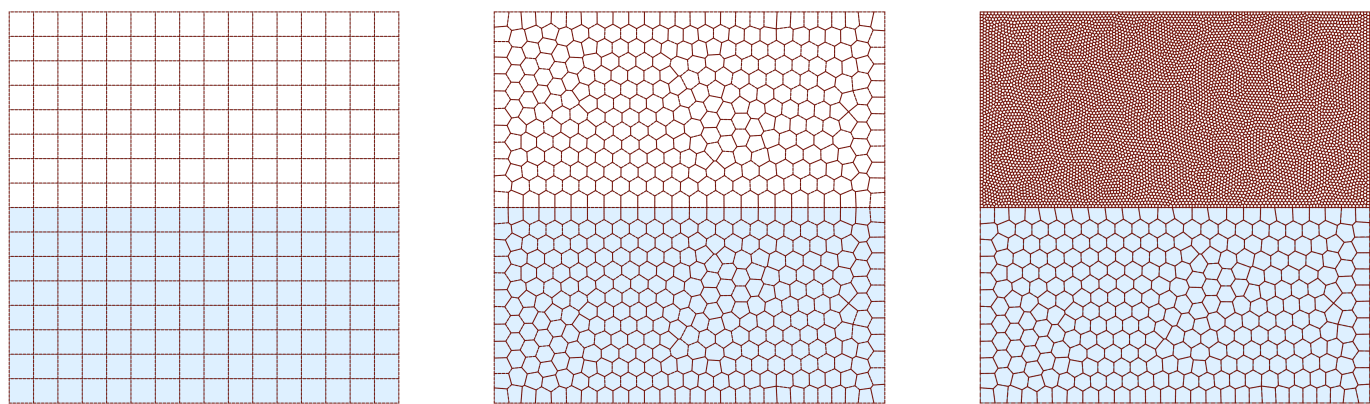

Figure 16: Computational domain and meshes composed of quadrilaterals (left panel), polygons (central panel) and polygons with hanging nodes at the interface (right panel).

The second test case deals with the propagation of an initial Gaussian-like pulse through a two-dimensional heterogeneous domain. The spatial domain is partitioned into $\bar{\Omega}=\bar{\Omega}_{1} \cup \bar{\Omega}_{2}$ with $\Omega_{1}:=(0,1) \times(0,0.5)$ and $\Omega_{2}:=(0.0,1) \times(0.5,1)$. As shown in Figure 16, three types of meshes are used, composed of either quadrilaterals or polygons. The actual meshes corresponding to the left and central panels used in the computations are finer. The meshes are fitted to the partition of the computational domain, and the subdomain $\Omega_{1}$ is shown in light blue color. The number of cells is 16,348 for the two meshes without hanging nodes (corresponding to $h:=2^{-7}$ for the quadrilateral mesh) and 8,512 for the polygonal mesh with hanging nodes (the maximum ratio between the largest and smallest edge of a cell is 6 ). The final time is $T_{\mathrm{f}}:=\frac{1}{4}$, and the wave speeds are $c_{1}:=5$ and $c_{2}:=1$. The source term is $f:=0$, and the initial condition is $p_{0}:=0$ and

$$
v_{0}(x, y):=-\frac{4}{10} \sqrt{\frac{10}{3}}\left(1600 r^{2}-1\right) \pi^{-\frac{1}{4}} \exp \left(-800 r^{2}\right),
$$

with $r^{2}:=\left(x-x_{c}\right)^{2}+\left(y-y_{c}\right)^{2}, x_{c}:=\frac{1}{2}$, and $y_{c}:=\frac{1}{4}$. Owing to the shape of $v_{0}$, the initial profile leads to a so-called Ricker (or Mexican hat) wavelet. Dirichlet boundary conditions are enforced over the whole boundary of $\Omega$, so that the wave propagates inside the domain and is reflected at the boundary. There is no analytical solution available with such boundary conditions.

We compare the results obtained with the HHO-Newmark, HHO-SDIRK $(3,4)$, and HHOERK(4) schemes. In Figure 17, we evaluate the influence of the discretization parameters for the HHO-Newmark scheme by reporting the velocity profiles as a function of time at two sensors, located at the points with coordinates $\left(\frac{1}{2}, \frac{1}{3}\right)$ and $\left(\frac{1}{2}, \frac{2}{3}\right)$. We consider the mixed-order setting and recall that $\tau_{\partial T}=\mathcal{O}\left(h_{T}^{-1}\right)$ for the HHO-Newmark scheme. We can see from the upper row in Figure 17 that the influence of the time step disappears for $\Delta t=0.025 \times 2^{-8}$ and from the central row that the influence of the polynomial degree disappears for $k=6$. The bottom row shows that the predictions on the quadrilateral mesh and the polygonal mesh overlap (recall that both meshes have the same number of cells). A similar study is performed in Figure 18 for the HHO-SDIRK $(3,4)$ scheme with the mixed-order setting and $\tilde{\tau}_{\partial T}=\frac{1}{\rho c} \frac{\ell_{\Omega}}{h_{T}}$. We can see from the upper row in Figure 18 that the influence of the time step disappears for $\Delta t=0.025 \times 2^{-6}$ (four times larger than for HHO-Newmark) and from the central row that the influence of the polynomial degree disappears for $k=5$ (one order lower than for HHONewmark). The bottom row shows that the predictions on the quadrilateral mesh and the polygonal mesh overlap (as for HHO-Newmark). Figure 19 displays the two-dimensional velocity distributions predicted by the $\operatorname{HHO} \operatorname{SDIRK}(3,4)$ scheme on the polygonal mesh (with hanging nodes) at the times $t \in\{0.015625,0.03125,0.25\}$. We see that the simulation captures well the numerous reflections of the initial pulse at the boundary as well as the penetration of the wave 

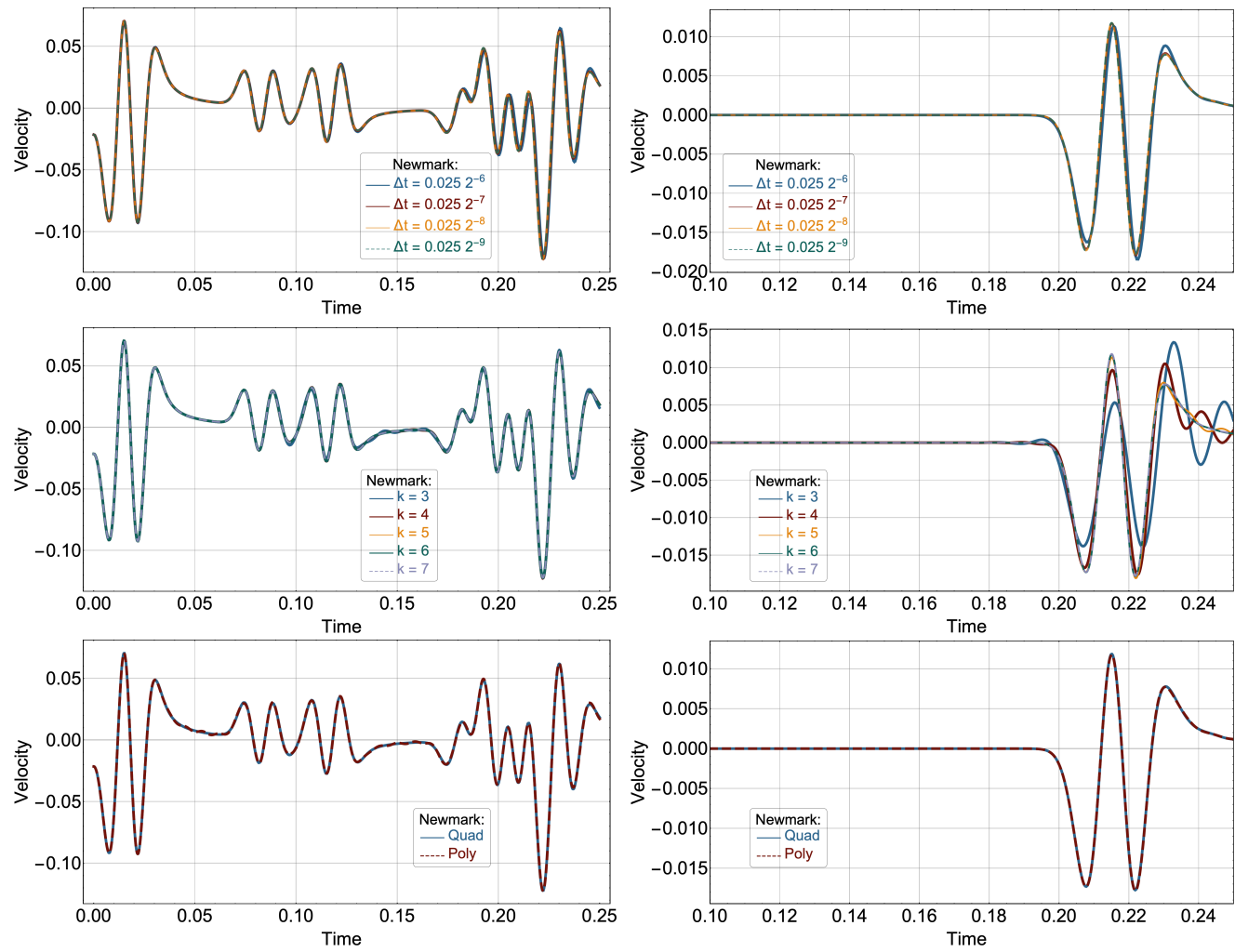

Figure 17: HHO-Newmark scheme: velocity as a function of time predicted at sensors located at the points with coordinates $\left(\frac{1}{2}, \frac{1}{3}\right)$ (left panel) and $\left(\frac{1}{2}, \frac{2}{3}\right)$ (right panel). Upper row: time steps $\Delta t \in 0.025 \times\left\{2^{-6}, 2^{-7}, 2^{-8}, 2^{-9}\right\}, k=6$, polygonal mesh. Central row: polynomial degrees $k \in\{3, \ldots, 7\}, \Delta t=0.025 \times 2^{-8}$, polygonal mesh. Bottom row: comparison of quadrilateral and polygonal mesh with hanging nodes, $\Delta t=0.025 \times 2^{-8}, k=6$.

into the upper subdomain. The velocity distributions are very similar to those predicted on the quadrilateral mesh (not shown for brevity), thereby confirming once again the capability of the scheme to handle polygonal meshes with hanging nodes, as motivated for instance by the need to use finer meshes on one subdomain due to the contrasted material properties. Furthermore, in Figure 20, we compare the relative energy loss as a function of time for the HHO-Newmark, HHO-ERK(4) (with $\tilde{\tau}_{\partial T}=\mathcal{O}(1)$ and $\Delta t=0.025 \times 2^{-9}$ ), and $\operatorname{HHO-SDIRK}(3,4)$ schemes. As expected, the HHO-Newmark scheme delivers exact energy balance up to machine error, whereas the relative energy loss incurred by the HHO-ERK(4) and HHO-SDIRK $(3,4)$ schemes is of the order of less than a percent at the final time. 

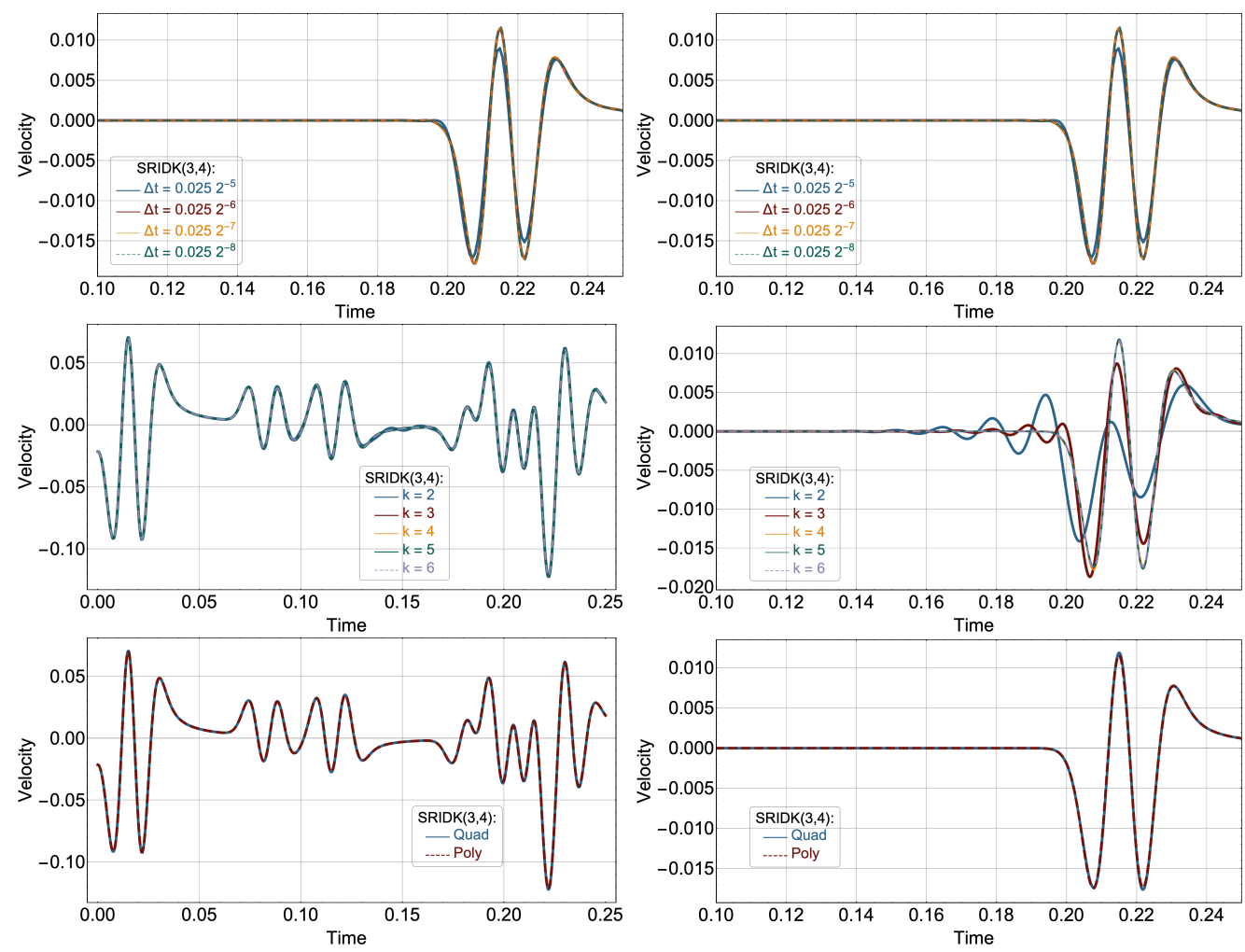

Figure 18: HHO-SDIRK $(3,4)$ scheme: velocity as a function of time predicted at sensors located at the points with coordinates $\left(\frac{1}{2}, \frac{1}{3}\right)$ (left panel) and $\left(\frac{1}{2}, \frac{2}{3}\right)$ (right panel). Upper row: time steps $\Delta t \in 0.025 \times\left\{2^{-5}, 2^{-6}, 2^{-7}, 2^{-8}\right\}, k=5$, polygonal mesh. Central row: polynomial degrees $k \in\{2, \ldots, 6\}, \Delta t=0.025 \times 2^{-6}$, polygonal mesh. Bottom row: comparison of quadrilateral and polygonal mesh with hanging nodes, $\Delta t=0.025 \times 2^{-6}, k=5$.
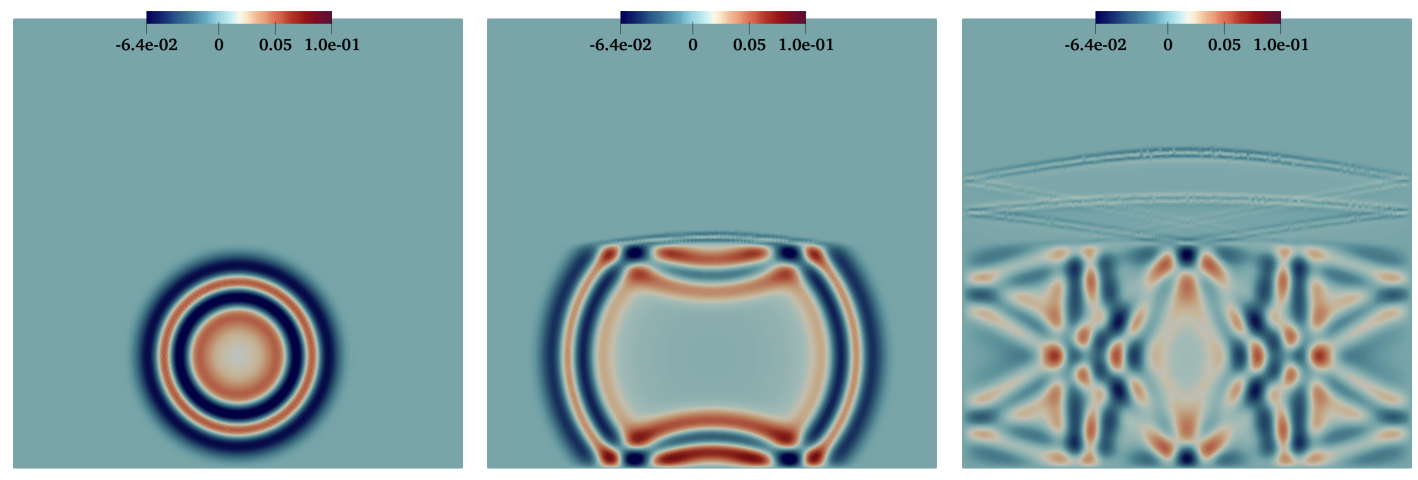

Figure 19: Two-dimensional velocity distributions predicted by the HHO-SDIRK $(3,4)$ scheme on the polygonal mesh (with hanging nodes) at the times $t \in\{0.015625,0.03125,0.25\}$ (from left to right); $k=5$ and $\tilde{\tau}_{\partial T}=\frac{1}{\rho c} \frac{\ell_{\Omega}}{h_{T}}$. 

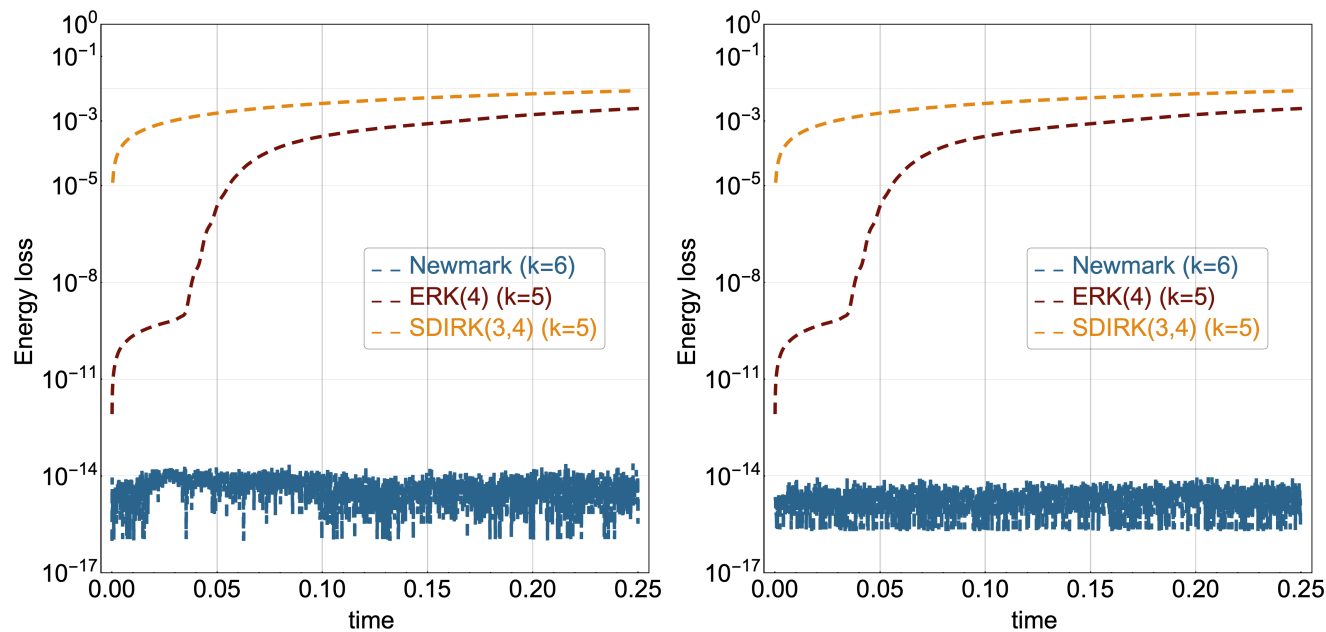

Figure 20: Relative energy loss as a function of time for HHO-Newmark $\left(\Delta t:=0.025 \times 2^{-8}\right)$, $\operatorname{HHO-ERK}(4)\left(\Delta t:=0.025 \times 2^{-9}, \tilde{\tau}_{\partial T}=\mathcal{O}(1)\right)$, and $\operatorname{HHO}-\operatorname{SDIRK}(3,4)\left(\Delta t:=0.025 \times 2^{-6}\right.$, $\tilde{\tau}_{\partial T}=\mathcal{O}\left(h_{T}^{-1}\right)$ ) on the quadrilateral (left panel) and polygonal (right panel, no hanging nodes) meshes. 


\begin{tabular}{|c|c|c|c|c|c|c|c|c|c|c|c|}
\hline scheme & $\left(k^{\prime}, k\right)$ & stab & solver & steps & \multicolumn{2}{|c|}{ t/step } & \multicolumn{2}{|c|}{ total } & \multicolumn{2}{|c|}{ it/sol } & err \\
\hline ERK(4) & $(6,5)$ & $\mathcal{O}(1)$ & $\mathrm{n} / \mathrm{a}$ & 5120 & \multicolumn{2}{|c|}{0.410} & \multicolumn{2}{|c|}{2099} & \multicolumn{2}{|c|}{$\overline{\mathrm{n} / \mathrm{a}}$} & 2.23 \\
\hline Newmark & $(7,6)$ & $\mathcal{O}\left(h_{T}^{-1}\right)$ & iter & 2560 & 22.76 & $\overline{1.013}$ & 58,265 & 2593 & 651 & 8 & 2.15 \\
\hline $\operatorname{SDIRK}(3,4)$ & $(6,5)$ & $\mathcal{O}\left(h_{T}^{-1}\right)$ & iter & 640 & 8.811 & 3.582 & 5639 & 2292 & 53 & 5 & 2.21 \\
\hline $\operatorname{SDIRK}(3,4)$ & $(5,5)$ & $\mathcal{O}\left(h_{T}^{-1}\right)$ & iter & 640 & 8.353 & 3.648 & 5345 & 2334 & 64 & 6 & 1.84 \\
\hline $\operatorname{SDIRK}(3,4)$ & $(6,5)$ & $\mathcal{O}(1)$ & iter & 640 & 3.438 & 2.779 & 2200 & 1778 & 20 & 3 & 4.45 \\
\hline $\operatorname{SDIRK}(3,4)$ & $(5,5)$ & $\mathcal{O}(1)$ & iter & 640 & 5.341 & 2.896 & 3418 & 1853 & 28 & 4 & 3.93 \\
\hline Newmark & $\overline{(\overline{(7,6)}}$ & $\overline{\overline{\mathcal{O}}\left(h_{T}^{-1}\right)}$ & direct & $\overline{2560}$ & \multicolumn{2}{|c|}{$\overline{0.515}$} & \multicolumn{2}{|c|}{$\overline{c 1318}$} & \multicolumn{2}{|c|}{$\mathrm{n} / \mathrm{a}$} & 2.15 \\
\hline $\operatorname{SDIRK}(3,4)$ & $(6,5)$ & $\mathcal{O}\left(h_{T}^{-1}\right)$ & direct & 640 & \multicolumn{2}{|c|}{1.579} & \multicolumn{2}{|c|}{1010} & \multicolumn{2}{|c|}{$\mathrm{n} / \mathrm{a}$} & 2.21 \\
\hline $\operatorname{SDIRK}(3,4)$ & $(5,5)$ & $\mathcal{O}\left(h_{T}^{-1}\right)$ & direct & 640 & \multicolumn{2}{|c|}{1.464} & \multicolumn{2}{|c|}{936} & \multicolumn{2}{|c|}{$\mathrm{n} / \mathrm{a}$} & 1.84 \\
\hline$\overline{\operatorname{SDIRK}(3,4)}$ & $(6,6)$ & $\mathcal{O}\left(h_{T}^{-1}\right)$ & direct & 2560 & \multicolumn{2}{|c|}{1.945} & \multicolumn{2}{|c|}{4979} & \multicolumn{2}{|c|}{$\mathrm{n} / \mathrm{a}$} & $\mathrm{n} / \mathrm{a}$ \\
\hline
\end{tabular}

Table 2: Information on CPU times, linear solve efficiency, and number of time steps for the HHO-Newmark, HHO-ERK(4) and HHO-SDIRK $(3,4)$ schemes on the polygonal mesh without hanging nodes. The column labeled "steps" reports the number of time steps needed to reach the final time, the column labeled "t/step" the CPU time per time step [s], the column labeled "total" the total CPU time, the column labeled "it/sol" the number of linear solver iterations per linear solve, and the column labeled "err" the relative maximum error at the location $\left(\frac{1}{2}, \frac{2}{3}\right)$ over the time nodes. In the columns "t/step", "total", and "it/sol", the first sub-column is for diagonal preconditioner and the second one for incomplete Cholesky/LU preconditioner. To compute the error in the last column, we use the scheme from the last row of the table as the reference solution (with the time step set to $\Delta t:=0.025 \times 2^{-8}$ ), which provides the reference value 0.0177 to normalize the errors.

Finally, Table 2 reports some information on the CPU time and linear solve efficiency for the various schemes on the polygonal mesh. The executions were performed on a 8-core CPU (2.7 GHz Quad-Core Intel Core i7) with LPDDR3 memory (16 GB $2133 \mathrm{MHz}$ ). All the implementations have been compiled with clang including the optimization level -03. The PARDISO sparse linear solver (Intel MKL library) was employed for the direct solver and Eigen library implementations of CG (HHO-Newmark) and BiCGStab (HHO-ERK(4) and HHO-SDIRK $(3,4)$ ) for the iterative solvers. For HHO-Newmark, we consider $\Delta t:=0.025 \times 2^{-8}$ and $\left(k^{\prime}, k\right)=(7,6)$, whereas for $\operatorname{HHO} \operatorname{SDIRK}(3,4)$, we consider $\Delta t:=0.025 \times 2^{-6}$ and either $\left(k^{\prime}, k\right)=(6,5)$ or $(5,5)$. For HHO-ERK $(4)$, we consider $\left(k^{\prime}, k\right)=(6,5)$, but the value of the time step depends on the CFL restriction. Our numerical experiments on this two-dimensional heterogeneous wave propagation problem give $\Delta t:=0.025 \times 2^{-9}$ for $\tilde{\tau}_{\partial T}=\frac{1}{\rho c}$. Notice that HHO-Newmark leads to 929,016 degrees of freedom (339,192 after static condensation), whereas HHO-ERK(4) and HHO$\operatorname{SDIRK}(3,4)$ lead to $1,191,856$ unknowns $(290,736$ for $\operatorname{HHO-SDIRK}(3,4)$ after static condensation). Concerning the implicit schemes, we observe that there is one solve for HHO-Newmark and three solves for $\mathrm{HHO} \operatorname{SDIRK}(3,4)$. If enough memory space is available, a direct solver can be considered (the factorization is preprocessed and stored once and for all), but otherwise an iterative solver has to be employed (the tolerance is set to $10^{-4}$ in our simulations). In this case, two preconditioners are considered, either diagonal preconditioning or incomplete factorization (Cholesky for HHO-Newmark and LU for HHO-SDIRK $(3,4)$ ).

The comparison of the various rows of Table 2 leads to the following conclusions. (i) If direct solvers are allowed, the lowest overall runtime is achieved by $\operatorname{HHO}-\operatorname{SDIRK}(3,4)$. (ii) $\mathrm{HHO}$ $\operatorname{SDIRK}(3,4)$ performs better than HHO-Newmark; this is in part due to the higher polynomial degree required for HHO-Newmark. (iii) For both implicit schemes, incomplete factorization preconditioners lead to better performances than diagonal preconditioners. We also mention that 


\begin{tabular}{|c|c|c|c|c|c|c|c|c|c|c|c|}
\hline scheme & $\left(k^{\prime}, k\right)$ & stab & solver & steps & \multicolumn{2}{|c|}{ t/step } & \multicolumn{2}{|c|}{ total } & \multicolumn{2}{|c|}{$\mathrm{it} / \mathrm{sol}$} & err \\
\hline $\operatorname{ERK}(4)$ & $\overline{(2(6,5)}$ & $\mathcal{O}(1)$ & $\mathrm{n} / \mathrm{a}$ & 5120 & \multicolumn{2}{|c|}{0.416} & \multicolumn{2}{|c|}{2129} & \multicolumn{2}{|c|}{$\mathrm{n} / \mathrm{a}$} & 2.27 \\
\hline Newmark & $(7,6)$ & $\mathcal{O}\left(h_{T}^{-1}\right)$ & iter & 2560 & 4.375 & 3.769 & 11,200 & 9648 & 157 & 77 & 2.19 \\
\hline $\operatorname{SDIRK}(3,4)$ & $(6,5)$ & $\mathcal{O}\left(h_{T}^{-1}\right)$ & iter & 640 & 8.124 & 1.948 & 5199 & 1246 & 72 & 7 & 2.18 \\
\hline $\operatorname{SDIRK}(3,4)$ & $(5,5)$ & $\mathcal{O}\left(h_{T}^{-1}\right)$ & iter & 640 & 9.136 & 1.998 & 5847 & 1279 & 87 & 8 & 2.34 \\
\hline $\operatorname{SDIRK}(3,4)$ & $(6,5)$ & $\mathcal{O}(1)$ & iter & 640 & 2.247 & 1.118 & 1438 & 716 & 23 & 4 & 2.44 \\
\hline $\operatorname{SDIRK}(3,4)$ & $(5,5)$ & $\mathcal{O}(1)$ & iter & 640 & 8.712 & 1.782 & 5575 & 1140 & 104 & 5 & 2.31 \\
\hline Newmark & $(7,6)$ & $\mathcal{O}\left(h_{T}^{-1}\right)$ & direct & 2560 & \multicolumn{2}{|c|}{0.252} & \multicolumn{2}{|c|}{645} & \multicolumn{2}{|c|}{$\mathrm{n} / \mathrm{a}$} & 2.19 \\
\hline $\operatorname{SDIRK}(3,4)$ & $(6,5)$ & $\mathcal{O}\left(h_{T}^{-1}\right)$ & direct & 640 & \multicolumn{2}{|c|}{0.907} & \multicolumn{2}{|c|}{580} & \multicolumn{2}{|c|}{$\mathrm{n} / \mathrm{a}$} & 2.18 \\
\hline $\operatorname{SDIRK}(3,4)$ & $(5,5)$ & $\mathcal{O}\left(h_{T}^{-1}\right)$ & direct & 640 & \multicolumn{2}{|c|}{0.873} & \multicolumn{2}{|c|}{558} & \multicolumn{2}{|c|}{$\mathrm{n} / \mathrm{a}$} & 2.34 \\
\hline
\end{tabular}

Table 3: Information on CPU times, linear solve efficiency, and number of time steps for the HHONewmark, HHO-ERK(4) and HHO-SDIRK $(3,4)$ schemes on the polygonal mesh with hanging nodes. The meaning of the various columns is the same as in Table 2.

we tried using the solution from the previous time step as an initial guess, without achieving further gains. (iv) The performance of HHO-ERK(4) is comparable to that of $\operatorname{HHO}-\operatorname{SDIRK}(3,4)$ combined with an iterative solver and an incomplete LU preconditioner. Thus, despite the CFL restriction (see the first line of Table 2), the explicit scheme remains competitive; a similar conclusion was reached in [35] in the context of HDG-RK schemes. (v) The choice of the stabilization parameter $\left(\mathcal{O}(1)\right.$ vs. $\left.\mathcal{O}\left(h_{T}^{-1}\right)\right)$ and the choice of $k^{\prime}$ (equal vs. mixed-order settings) do not have a strong effect on the efficiency of the SDIRK schemes. We recommend the choice $\mathcal{O}\left(h_{T}^{-1}\right)$ for the stabilization parameter since it leads to better convergence rates in the $L^{2}$-norm for smooth solutions. Similar information on the computational efficiency is presented in Table 3 for the various schemes on the non-conforming polygonal mesh, leading to similar conclusions as those drawn above, though with a more pronounced advantage to $\operatorname{HHO}-\operatorname{SDIRK}(3,4)$ with either direct solvers or iterative solvers with incomplete LU factorization.

\section{Conclusions}

We have devised and studied hybrid-high order (HHO) methods for the acoustic wave equation in the time domain. HHO methods for space semi-discretization were combined with either energy-preserving Newmark schemes or with Runge-Kutta schemes for the time discretization. Our schemes are implicit in time if the second-order formulation of the wave equation is retained, whereas explicit time-marching schemes are possible by using the first-order (mixed) formulation and considering a mixed-order polynomial setting for the cell and the face unknowns. We have also bridged the present schemes to the literature on hybridizable discontinuous Galerkin (HDG) methods. Our numerical results indicate optimal convergence rates for the errors in the $H^{1}$-norm (and in the $L^{2}$-norm depending on the stabilization), support of polygonal meshes, and accurate handling of test cases with contrasted properties. Future work will be devoted to extend the present schemes to coupled problems combining acoustic and elastodynamic wave propagation.

\section{Acknowledgments}

The authors would like to thank L. Guillot (CEA/DAM) for insightful discussions and CEA/DAM for partial financial support. EB was partially supported by the EPSRC grants EP/P01576X/1 and $\mathrm{EP} / \mathrm{P} 012434 / 1$. 


\section{References}

[1] M. Abbas, A. Ern, and N. Pignet. Hybrid High-Order methods for finite deformations of hyperelastic materials. Comput. Mech., 62(4):909-928, 2018.

[2] M. Abbas, A. Ern, and N. Pignet. A Hybrid High-Order method for incremental associative plasticity with small deformations. Comput. Methods Appl. Mech. Engrg., 346:891-912, 2019 .

[3] J. B. Angel, J. W. Banks, and W. D. Henshaw. High-order upwind schemes for the wave equation on overlapping grids: Maxwell's equations in second-order form. J. Comput. Phys., 352:534-567, 2018.

[4] D. Appelö and T. Hagstrom. A new discontinuous Galerkin formulation for wave equations in second-order form. SIAM J. Numer. Anal., 53(6):2705-2726, 2015.

[5] B. Ayuso de Dios, K. Lipnikov, and G. Manzini. The nonconforming virtual element method. ESAIM Math. Model. Numer. Anal., 50(3):879-904, 2016.

[6] J. W. Banks, T. Hagstrom, and J. Jacangelo. Galerkin differences for acoustic and elastic wave equations in two space dimensions. J. Comput. Phys., 372:864-892, 2018.

[7] E. Bécache, P. Joly, and C. Tsogka. An analysis of new mixed finite elements for the approximation of wave propagation problems. SIAM J. Numer. Anal., 37(4):1053-1084, 2000.

[8] L. Botti, D. A. Di Pietro, and J. Droniou. A hybrid high-order method for the incompressible Navier-Stokes equations based on Temam's device. J. Comput. Phys., 376:786-816, 2019.

[9] M. Botti, D. A. Di Pietro, and P. Sochala. A Hybrid High-Order method for nonlinear elasticity. SIAM J. Numer. Anal., 55(6):2687-2717, 2017.

[10] E. Burman, O. Duran, A. Ern, and M. Steins. Convergence analysis of hybrid high-order methods for the wave equation. J. Sci. Comput., 2021. To appear, see https://hal . archives-ouvertes .fr/hal-02922720.

[11] E. Burman and A. Ern. An unfitted hybrid high-order method for elliptic interface problems. SIAM J. Numer. Anal., 56(3):1525-1546, 2018.

[12] E. Burman, A. Ern, and M. A. Fernández. Explicit Runge-Kutta schemes and finite elements with symmetric stabilization for first-order linear PDE systems. SIAM J. Numer. Anal., 48(6):2019-2042, 2010.

[13] V. Calo, M. Cicuttin, Q. Deng, and A. Ern. Spectral approximation of elliptic operators by the hybrid high-order method. Math. Comp., 88(318):1559-1586, 2019.

[14] K. L. Cascavita, J. Bleyer, X. Chateau, and A. Ern. Hybrid discretization methods with adaptive yield surface detection for Bingham pipe flows. J. Sci. Comput., 77(3):1424-1443, 2018.

[15] F. Chave, D. Di Pietro, and S. Lemaire. A three-dimensional Hybrid High-Order method for magnetostatics. Available at https://hal.archives-ouvertes.fr/hal-02407175, 2020. 
[16] C.-S. Chou, C.-W. Shu, and Y. Xing. Optimal energy conserving local discontinuous Galerkin methods for second-order wave equation in heterogeneous media. J. Comput. Phys., 272:88-107, 2014.

[17] E. T. Chung and B. Engquist. Optimal discontinuous Galerkin methods for wave propagation. SIAM J. Numer. Anal., 44(5):2131-2158, 2006.

[18] M. Cicuttin, D. A. Di Pietro, and A. Ern. Implementation of discontinuous skeletal methods on arbitrary-dimensional, polytopal meshes using generic programming. J. Comput. Appl. Math., 344:852-874, 2018.

[19] B. Cockburn, D. A. Di Pietro, and A. Ern. Bridging the hybrid high-order and hybridizable discontinuous Galerkin methods. ESAIM Math. Model Numer. Anal., 50(3):635-650, 2016.

[20] B. Cockburn, Z. Fu, A. Hungria, L. Ji, M. A. Sánchez, and F.-J. Sayas. Stormer-Numerov HDG methods for acoustic waves. J. Sci. Comput., 75(2):597-624, 2018.

[21] B. Cockburn, J. Gopalakrishnan, and R. Lazarov. Unified hybridization of discontinuous Galerkin, mixed, and continuous Galerkin methods for second order elliptic problems. SIAM J. Numer. Anal., 47(2):1319-1365, 2009.

[22] G. Cohen, P. Joly, J. E. Roberts, and N. Tordjman. Higher order triangular finite elements with mass lumping for the wave equation. SIAM J. Numer. Anal., 38(6):2047-2078, 2001.

[23] G. C. Cohen. Higher-Order Numerical Methods for Transient Wave Equations. Springer Berlin Heidelberg, 2002.

[24] D. A. Di Pietro, J. Droniou, and G. Manzini. Discontinuous Skeletal Gradient Discretisation Methods on polytopal meshes. J. Comput. Phys., 355:397-425, 2018.

[25] D. A. Di Pietro and A. Ern. A hybrid high-order locking-free method for linear elasticity on general meshes. Comput. Meth. Appl. Mech. Engrg., 283:1-21, 2015.

[26] D. A. Di Pietro, A. Ern, and S. Lemaire. An arbitrary-order and compact-stencil discretization of diffusion on general meshes based on local reconstruction operators. Comput. Meth. Appl. Math., 14(4):461-472, 2014.

[27] D. A. Di Pietro, A. Ern, A. Linke, and F. Schieweck. A discontinuous skeletal method for the viscosity-dependent Stokes problem. Comput. Methods Appl. Mech. Engrg., 306:175-195, 2016.

[28] A. Ezziani and P. Joly. Local time stepping and discontinuous Galerkin methods for symmetric first order hyperbolic systems. J. Comput. Appl. Math., 234(6):1886-1895, 2010.

[29] R. S. Falk and G. R. Richter. Explicit finite element methods for symmetric hyperbolic equations. SIAM J. Numer. Anal., 36(3):935-952, 1999.

[30] F. X. Giraldo and M. A. Taylor. A diagonal-mass-matrix triangular-spectral-element method based on cubature points. J. Engrg. Math., 56(3):307-322, 2006.

[31] R. Griesmaier and P. Monk. Discretization of the wave equation using continuous elements in time and a hybridizable discontinuous Galerkin method in space. J. Sci. Comput., 58(2):472498, 2014. 
[32] M. J. Grote, A. Schneebeli, and D. Schötzau. Discontinuous Galerkin finite element method for the wave equation. SIAM J. Numer. Anal., 44(6):2408-2431, 2006.

[33] J. S. Hesthaven and T. Warburton. Nodal high-order methods on unstructured grids. I. Time-domain solution of Maxwell's equations. J. Comput. Phys., 181(1):186-221, 2002.

[34] S. Krenk. Energy conservation in Newmark based time integration algorithms. Comput. Methods Appl. Mech. Engrg., 195(44-47):6110-6124, 2006.

[35] M. Kronbichler, S. Schoeder, C. Müller, and W. A. Wall. Comparison of implicit and explicit hybridizable discontinuous Galerkin methods for the acoustic wave equation. Internat. J. Numer. Methods Engrg., 106(9):712-739, 2016.

[36] J.-L. Lions and E. Magenes. Non-homogeneous boundary value problems and applications. Vols. I, II. Springer-Verlag, New York-Heidelberg, 1972. Translated from the French by P. Kenneth, Die Grundlehren der mathematischen Wissenschaften, Band 181-182.

[37] F. Marazzato, A. Ern, C. Mariotti, and L. Monasse. An explicit pseudo-energy conserving time-integration scheme for Hamiltonian dynamics. Comput. Methods Appl. Mech. Engrg., 347:906-927, 2019.

[38] P. Monk and G. R. Richter. A discontinuous Galerkin method for linear symmetric hyperbolic systems in inhomogeneous media. J. Sci. Comput., 22/23:443-477, 2005.

[39] N. C. Nguyen and J. Peraire. Hybridizable discontinuous Galerkin methods for partial differential equations in continuum mechanics. J. Comput. Phys., 231(18):5955-5988, 2012.

[40] N. C. Nguyen, J. Peraire, and B. Cockburn. High-order implicit hybridizable discontinuous Galerkin methods for acoustics and elastodynamics. J. Comput. Phys., 230(10):3695-3718, 2011.

[41] M. A. Sánchez, C. Ciuca, N. C. Nguyen, J. Peraire, and B. Cockburn. Symplectic Hamiltonian HDG methods for wave propagation phenomena. J. Comput. Phys., 350:951-973, 2017.

[42] B. Sjögreen and N. A. Petersson. A fourth order accurate finite difference scheme for the elastic wave equation in second order formulation. J. Sci. Comput., 52(1):17-48, 2012.

[43] M. Stanglmeier, N. C. Nguyen, J. Peraire, and B. Cockburn. An explicit hybridizable discontinuous Galerkin method for the acoustic wave equation. Comput. Methods Appl. Mech. Engrg., 300:748-769, 2016.

[44] C. Talischi, G. H. Paulino, A. Pereira, and I. F. M. Menezes. Polymesher: a general-purpose mesh generator for polygonal elements written in matlab. Struct. Multidisc. Optim., 45:309$328,2012$.

[45] K. Virta and K. Mattsson. Acoustic wave propagation in complicated geometries and heterogeneous media. J. Sci. Comput., 61(1):90-118, 2014. 